Electronic supporting information for

\title{
Stable M(II)-Radicals and Nickel(III) complexes of a bis(phenol) N-heterocyclic Carbene chelated to Group 10 Metal Ions
}

Romain Kunert, Christian Philouze, Olivier Jarjayes, * Fabrice Thomas*

Univ. Grenoble Alpes, CNRS, DCM, F-38000 Grenoble, France.

Email: fabrice.thomas@ univ-grenoble-alpes.fr.

\section{$\underline{\text { Content: }}$}

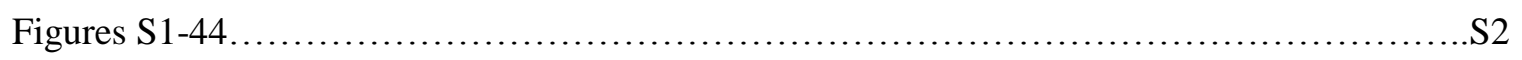

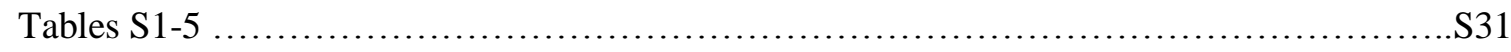

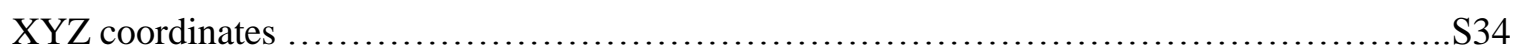

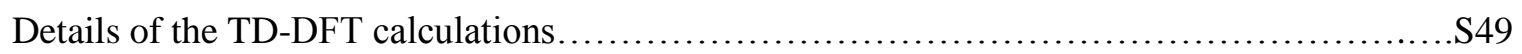


Figures

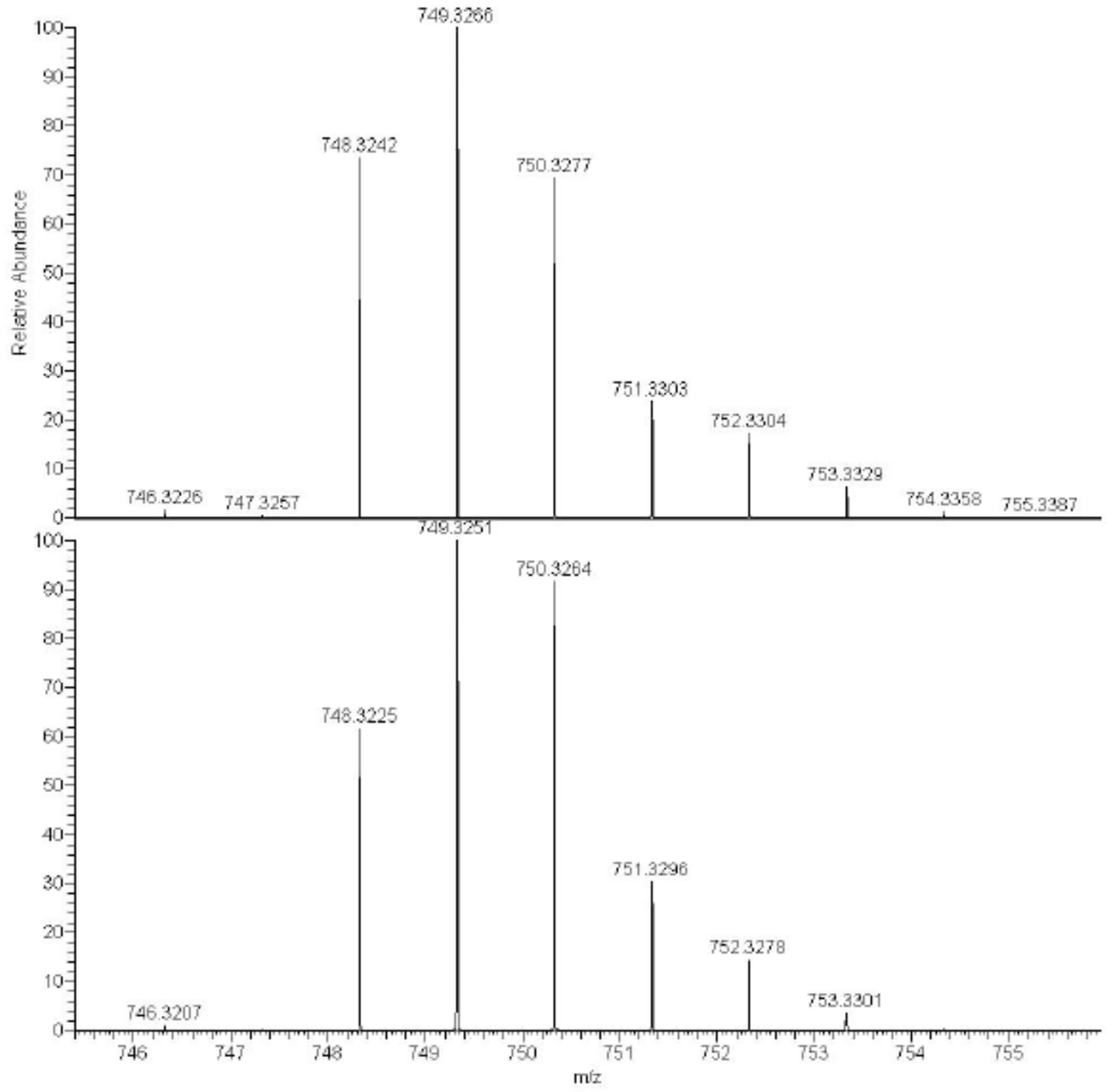

Figure S1. HRMS of $\mathrm{H}_{2} \mathrm{~L}^{\mathrm{C} 2 \mathrm{O} 2}$. Top: simulated spectrum; bottom, experimental spectrum. 


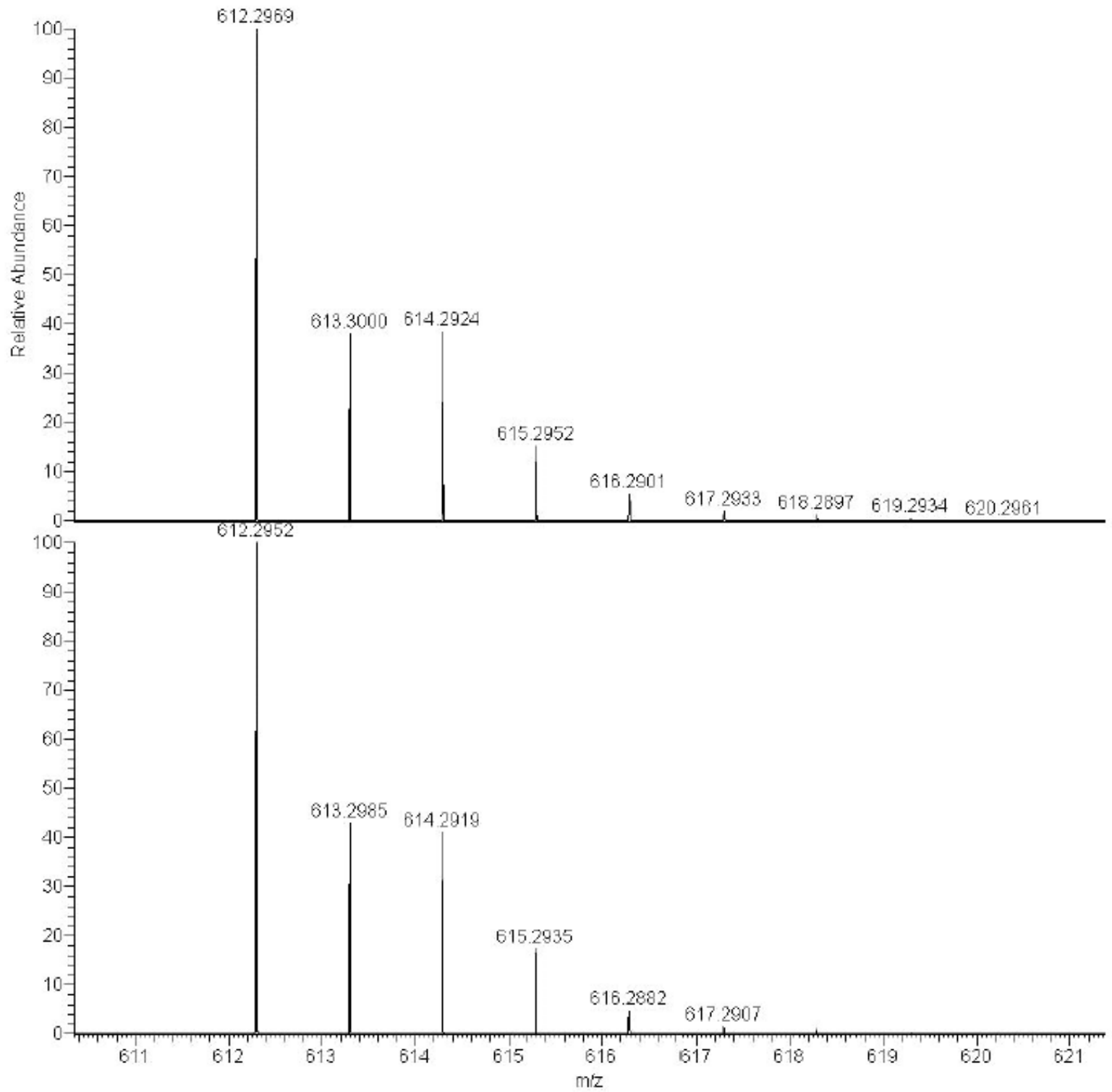

Figure S2. HRMS of 1. Top: simulated spectrum; bottom, experimental spectrum. 


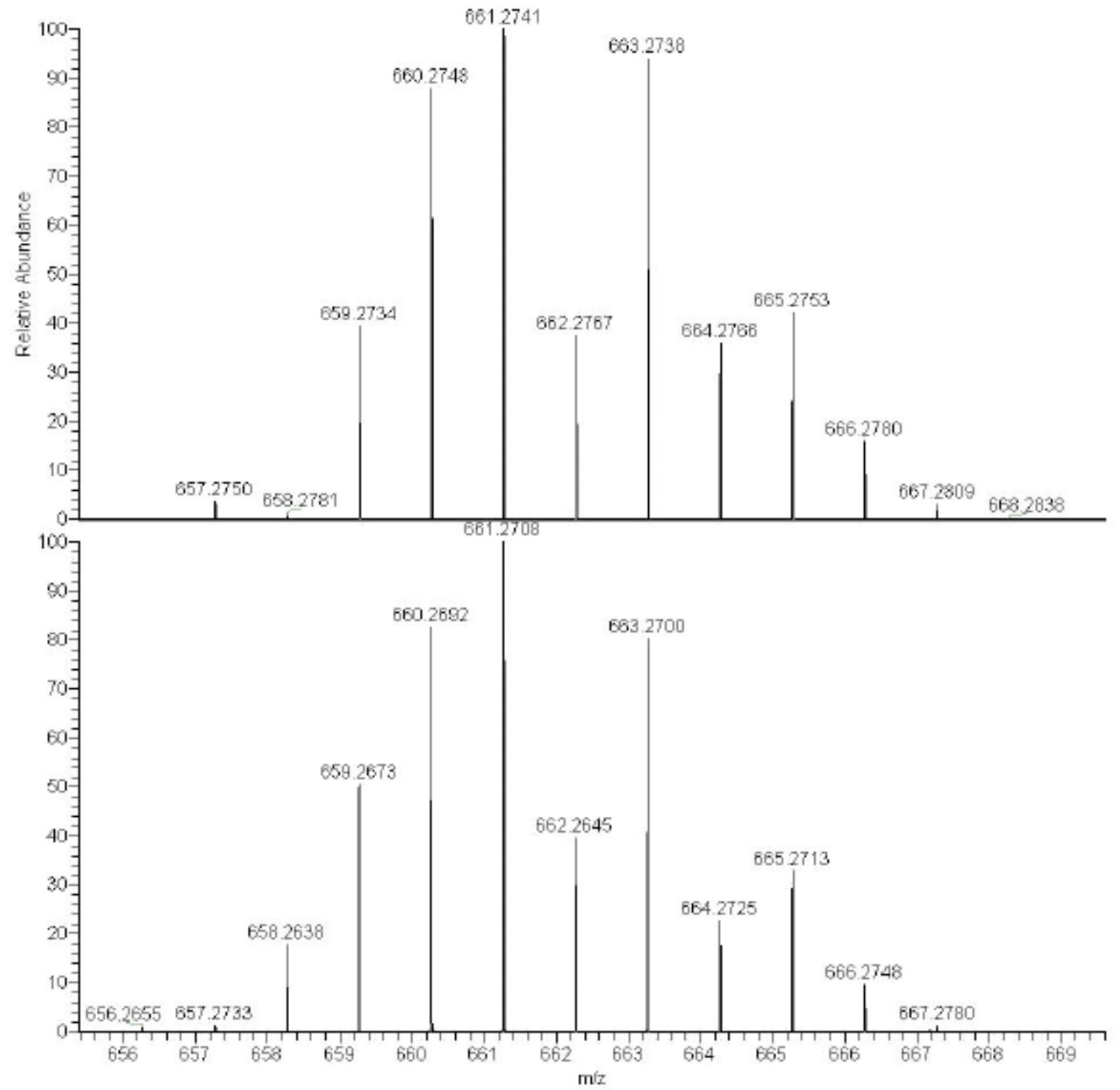

Figure S3. HRMS of 2. Top: simulated spectrum; bottom, experimental spectrum. 


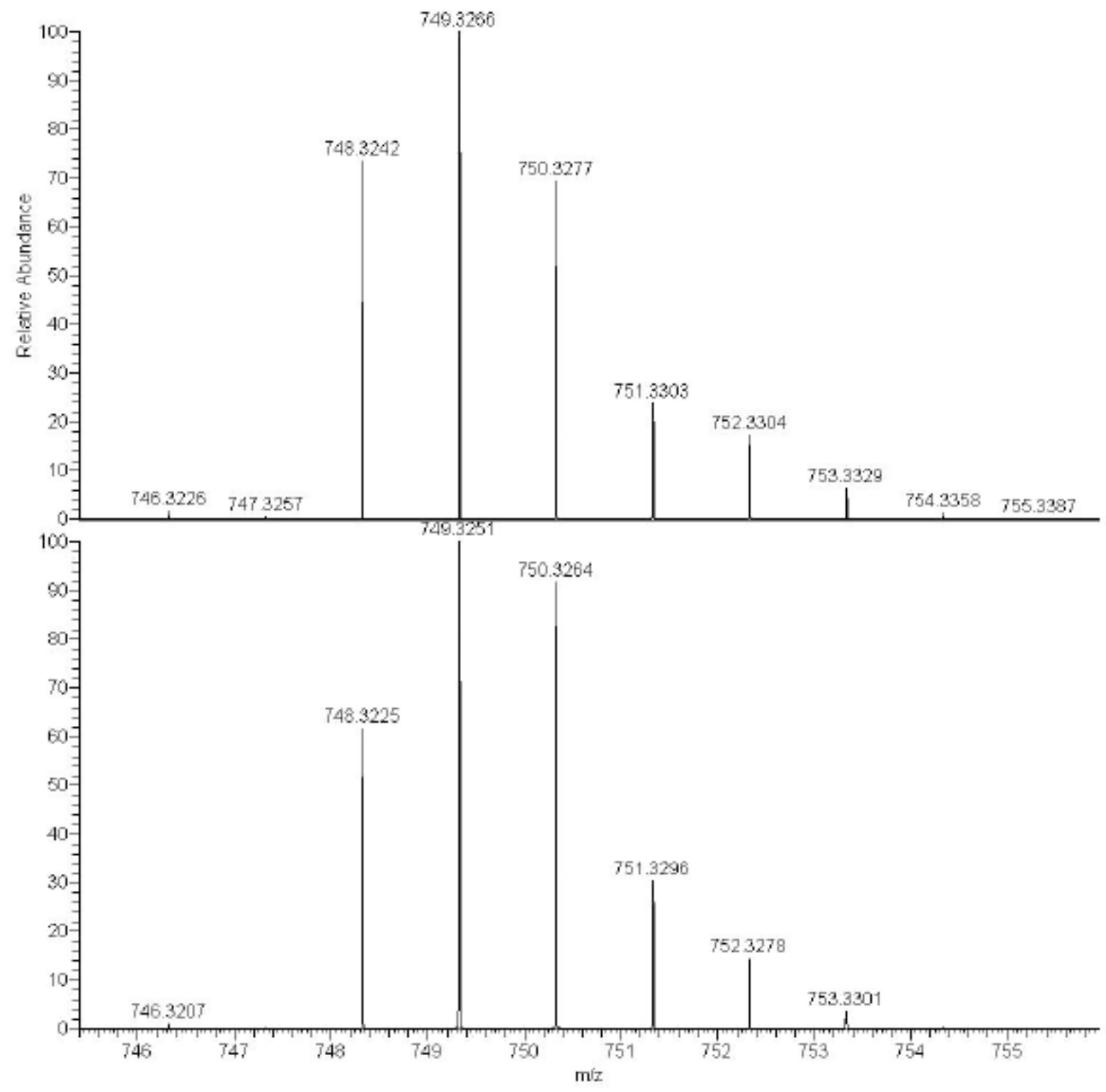

Figure S4. HRMS of 3. Top: simulated spectrum; bottom, experimental spectrum. 
@PROTON DMSO /opt/topspin cire 7

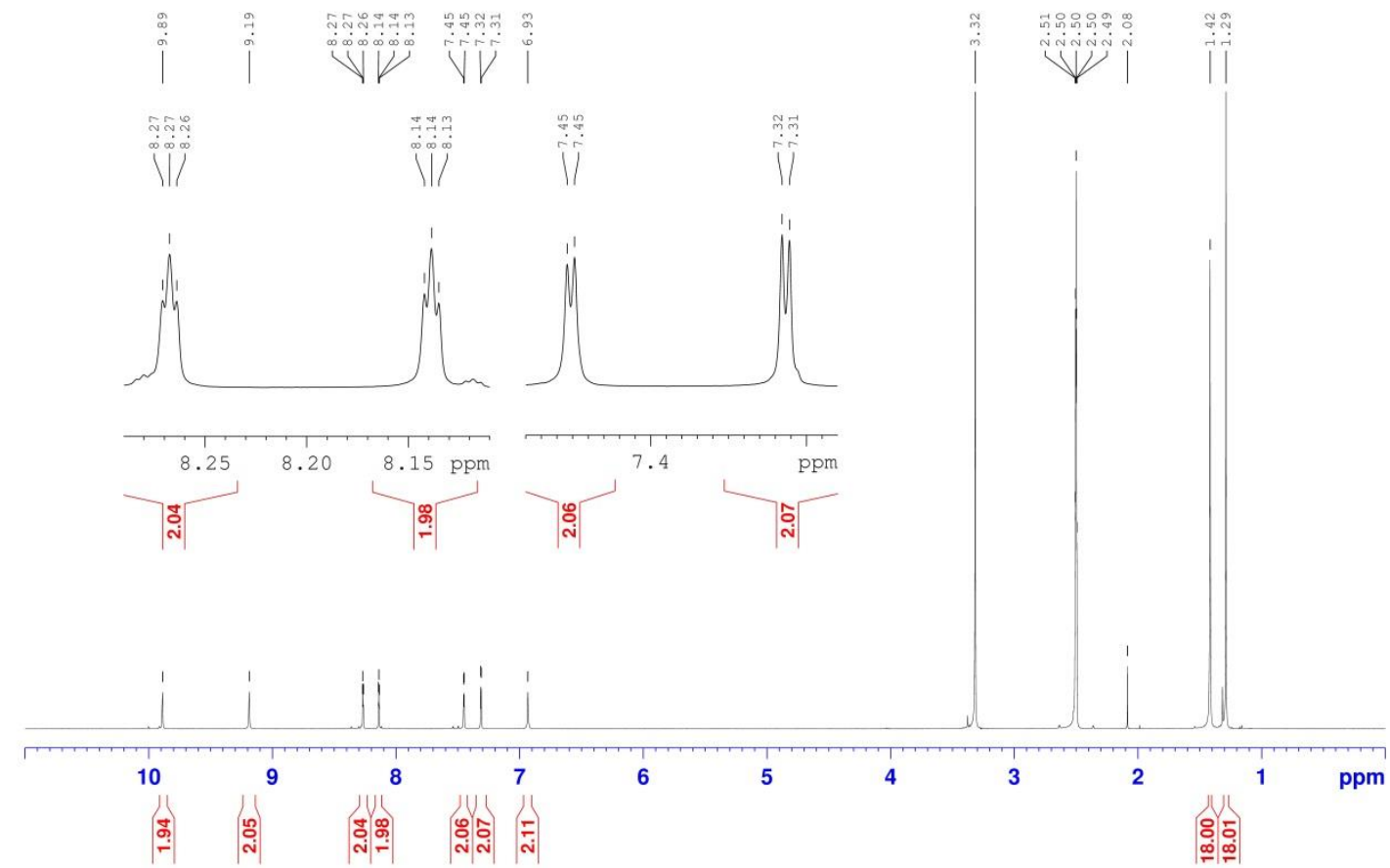

Figure S5. ${ }^{1} \mathrm{H}$ NMR spectrum $\left(\mathrm{d}_{6}-\mathrm{DMSO}\right)$ of $\mathrm{H}_{2} \mathrm{~L}^{\mathrm{C} 2 \mathrm{O} 2}$. 
@PROTON DMSO /opt/topspin cire 13

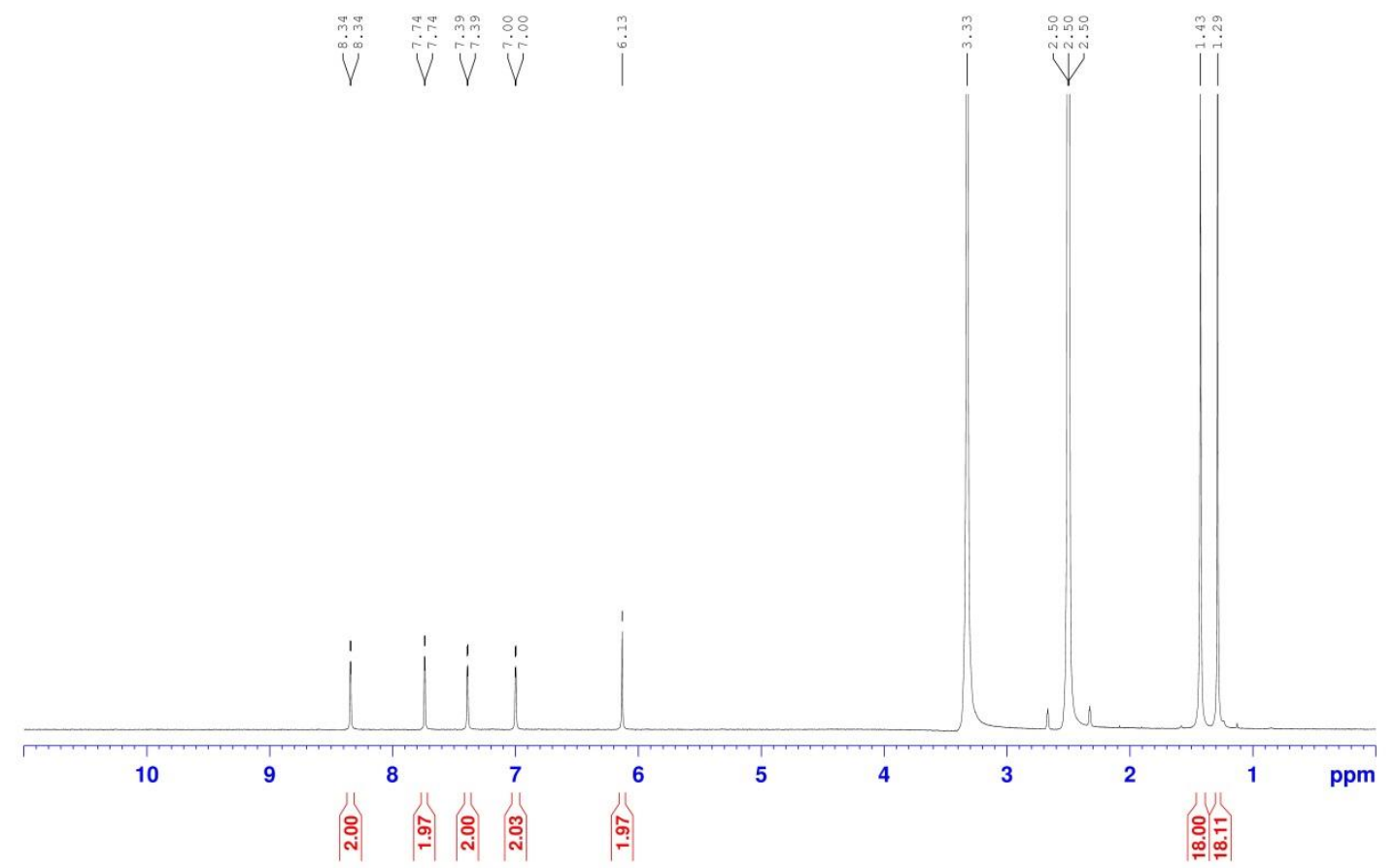

Figure S6. ${ }^{1} \mathrm{H}$ NMR spectrum $\left(\mathrm{d}_{6}-\mathrm{DMSO}\right)$ of $\mathbf{1}$. 
@PROTON DMSO /opt/topspin cire 24

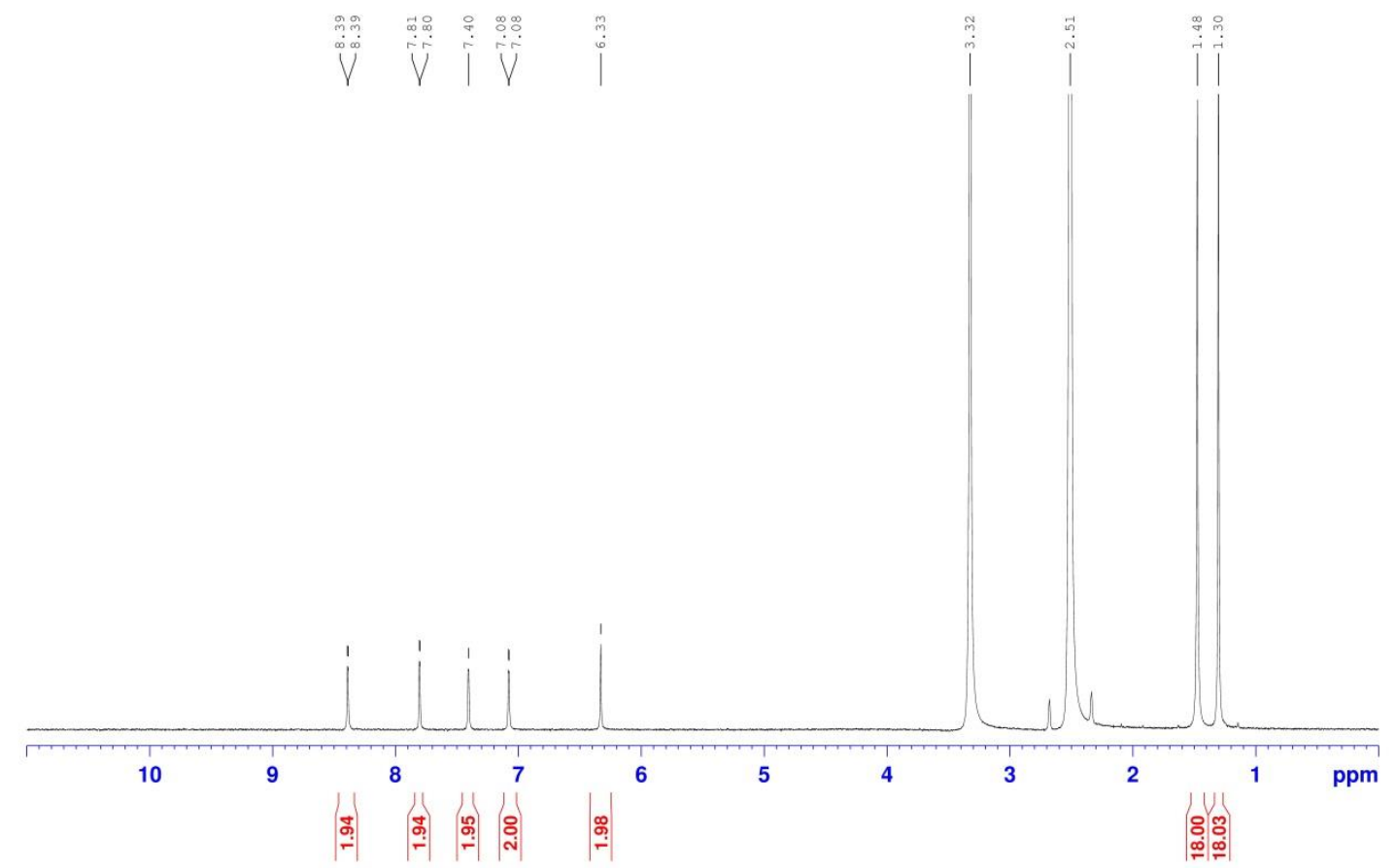

Figure S7. ${ }^{1} \mathrm{H}$ NMR spectrum $\left(\mathrm{d}_{6}\right.$-DMSO) of 2. 
@PROTON DMSO /opt/topspin cire 13

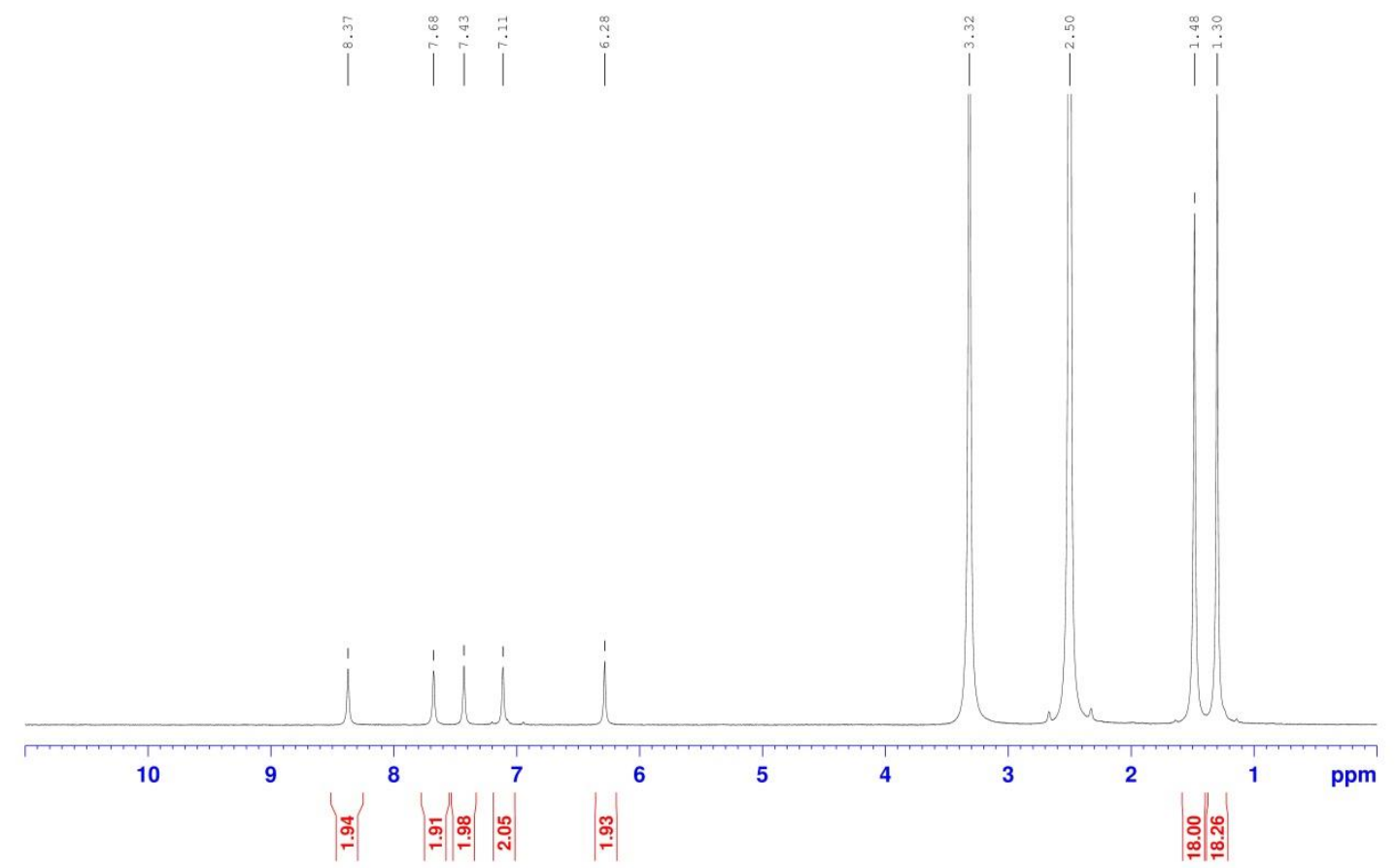

Figure S8. ${ }^{1} \mathrm{H}$ NMR spectrum $\left(\mathrm{d}_{6}-\mathrm{DMSO}\right)$ of $\mathbf{3}$. 
@CARBONE DMSO /opt/topspin cire 7

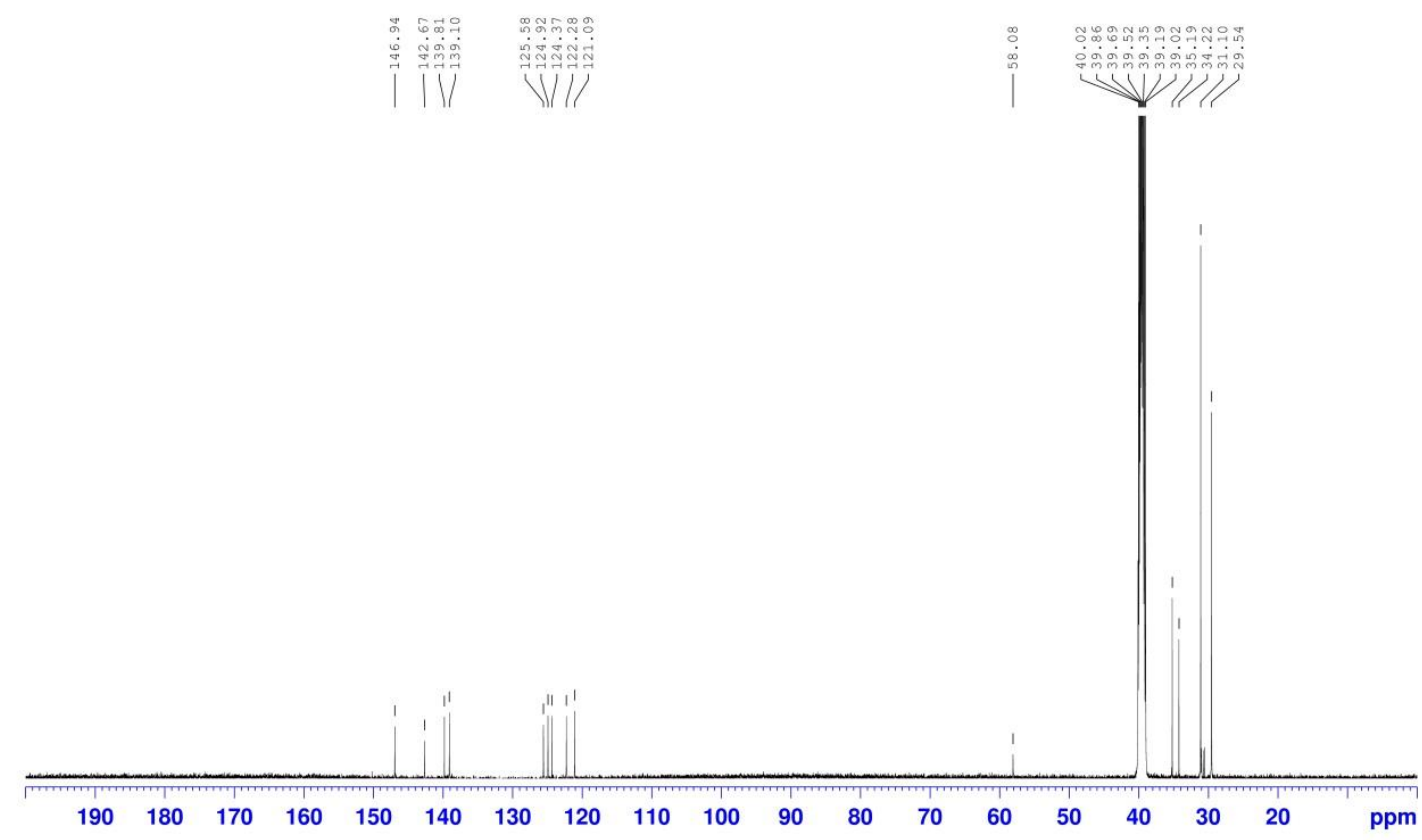

Figure S9. ${ }^{13} \mathrm{C}$ NMR spectrum $\left(\mathrm{d}_{6}\right.$-DMSO) of $\mathrm{H}_{2} \mathrm{~L}^{\mathrm{C} 2 \mathrm{O} 2}$. 
@CARBONE DMSO /opt/topspin cire 9

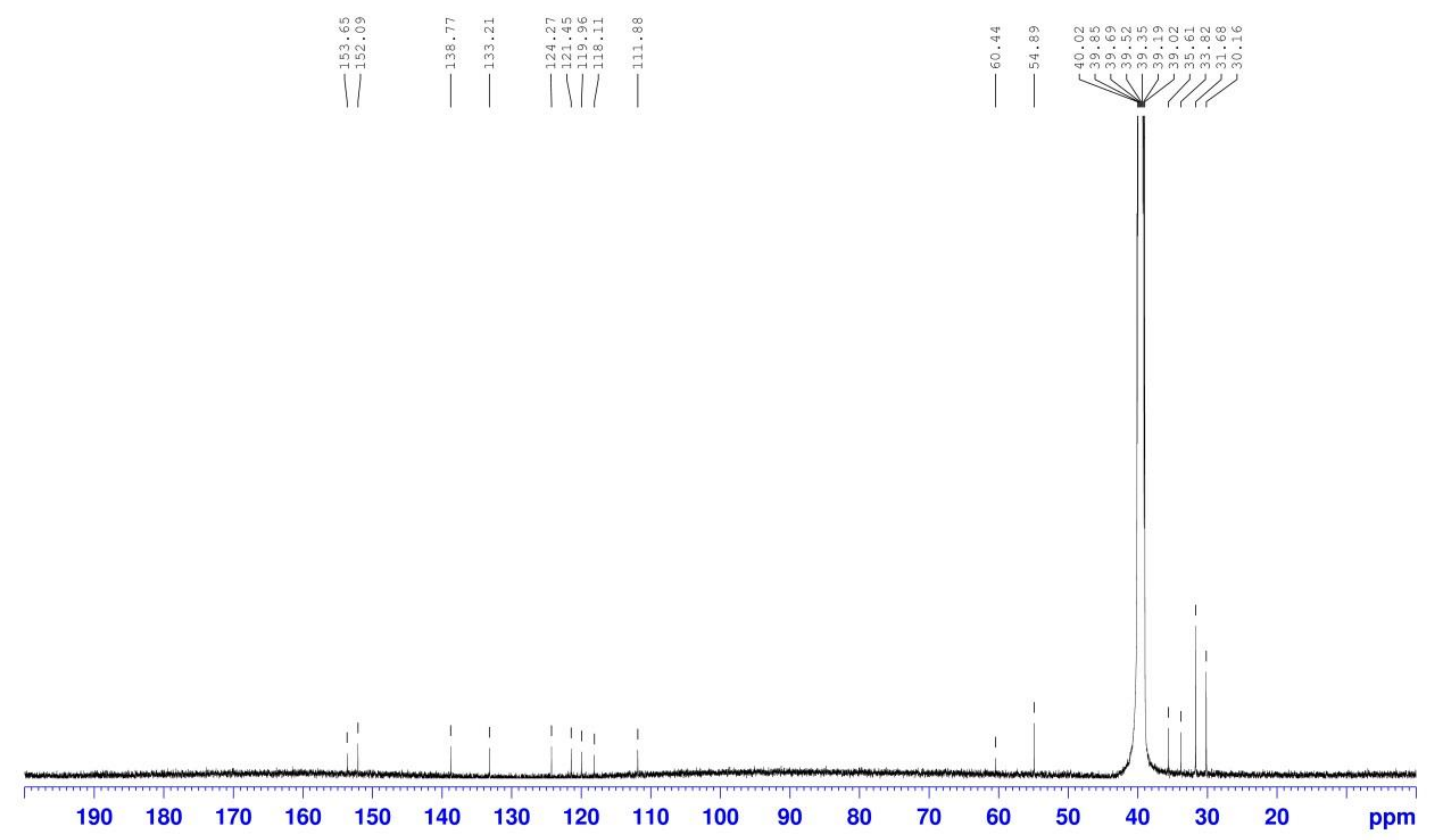

Figure S10. ${ }^{13} \mathrm{C}$ NMR spectrum $\left(\mathrm{d}_{6}\right.$-DMSO) of $\mathbf{1}$. 
@CARBONE DMSO /opt/topspin cire 8

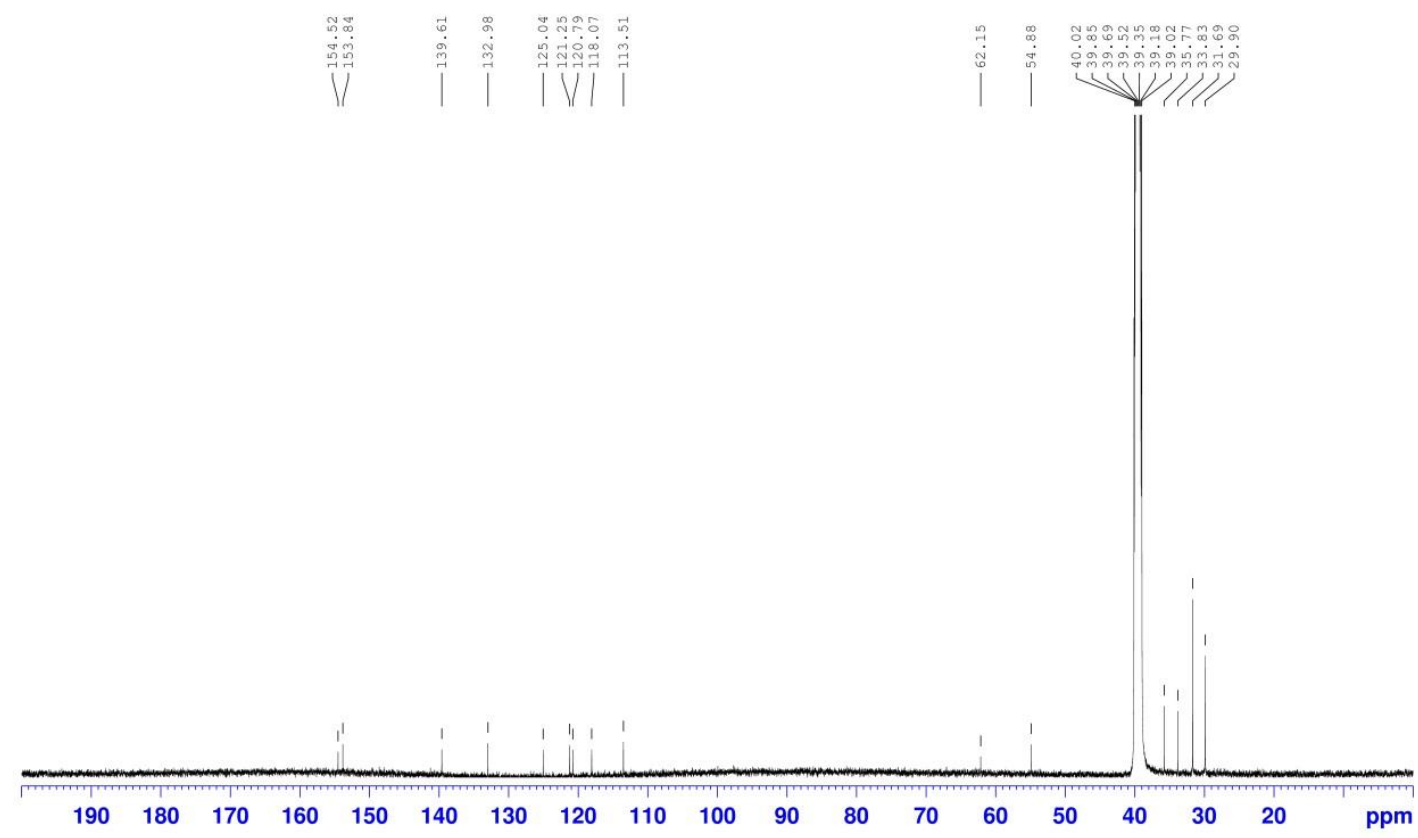

Figure S11. ${ }^{13} \mathrm{C}$ NMR spectrum $\left(\mathrm{d}_{6}\right.$-DMSO) of 2. 


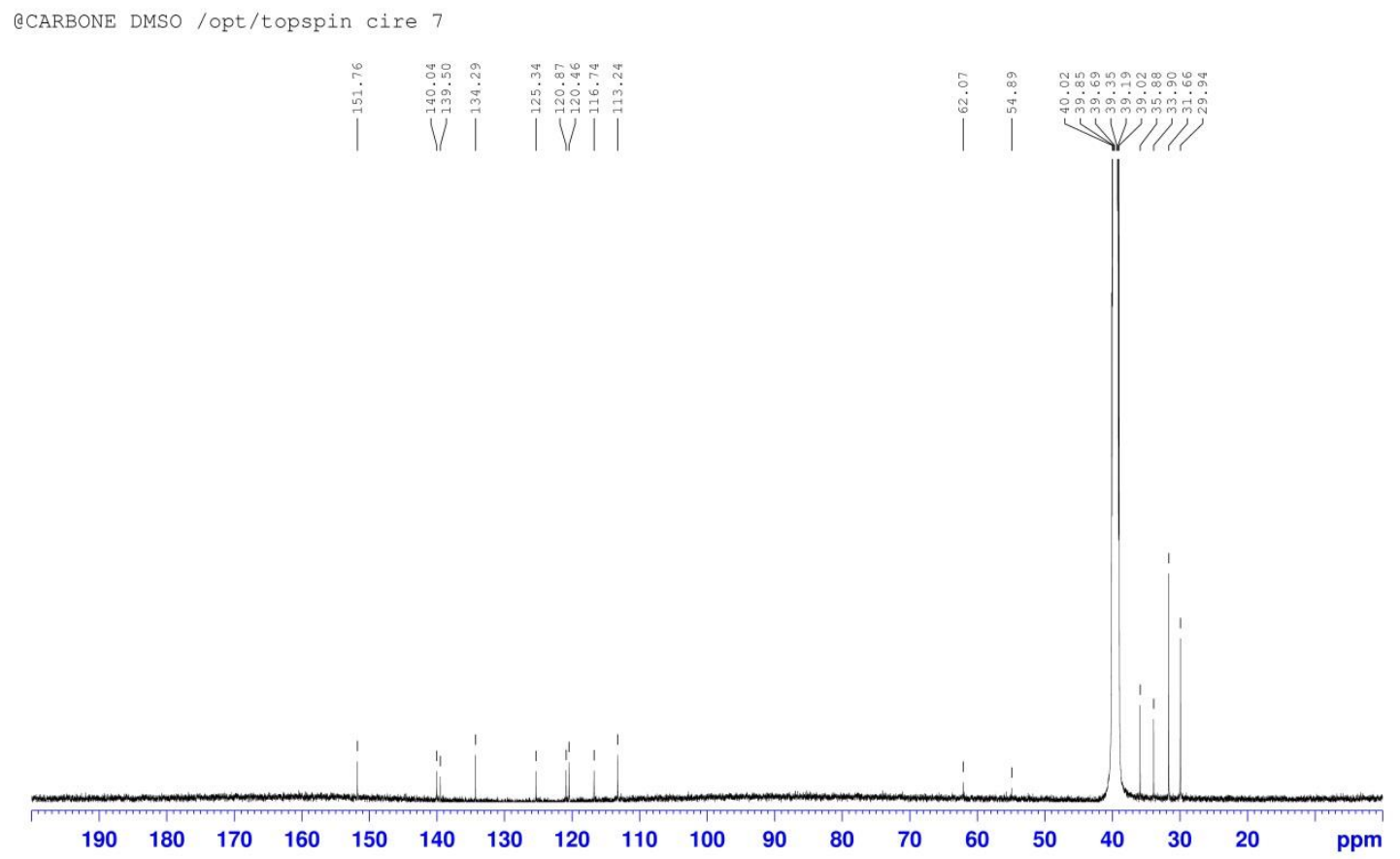

Figure S12. ${ }^{13} \mathrm{C}$ NMR spectrum $\left(\mathrm{d}_{6}-\mathrm{DMSO}\right)$ of $\mathbf{3}$.

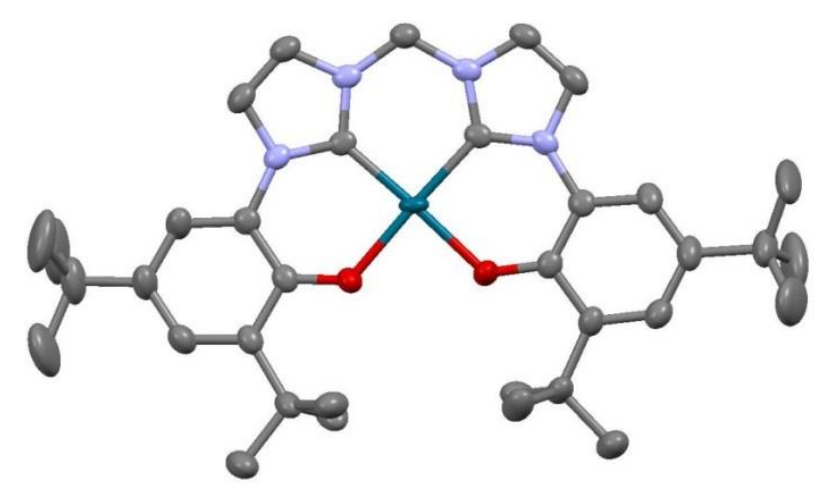

Figure S13. X-Ray crystal structure of 2 


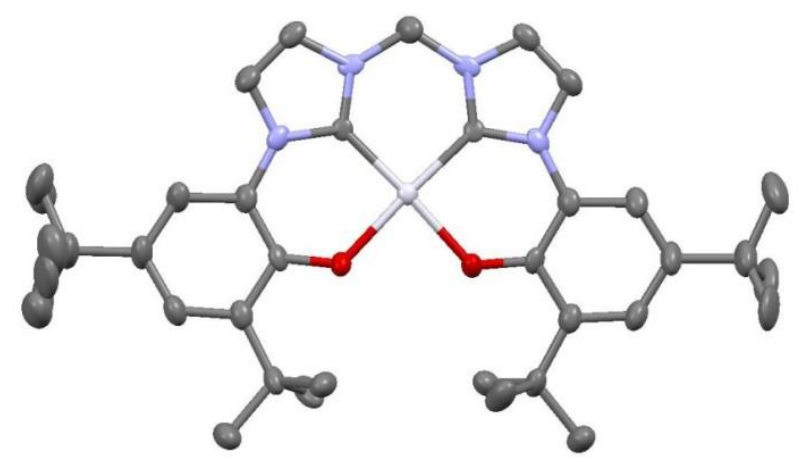

Figure S14. X-Ray crystal structure of 3

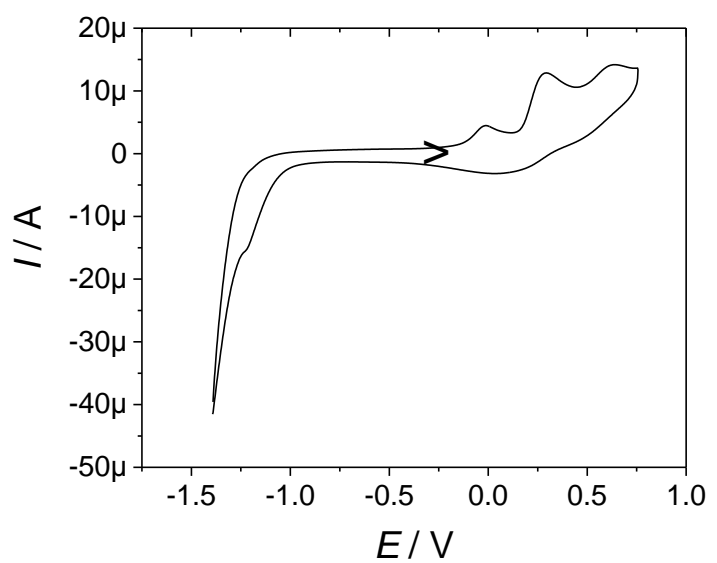

Figure S15. Cyclic voltammetry curves of $\mathrm{H}_{2} \mathrm{~L}^{\mathrm{C} 2 \mathrm{O} 2}$ in $0.5 \mathrm{mM} \mathrm{CH}_{2} \mathrm{Cl}_{2}$ solution containing $0.1 \mathrm{M}$ TBAP. $T=293 \mathrm{~K}$, vitreous carbon working electrode. The potentials are given versus the $\mathrm{Fc}^{+} / \mathrm{Fc}$ reference.
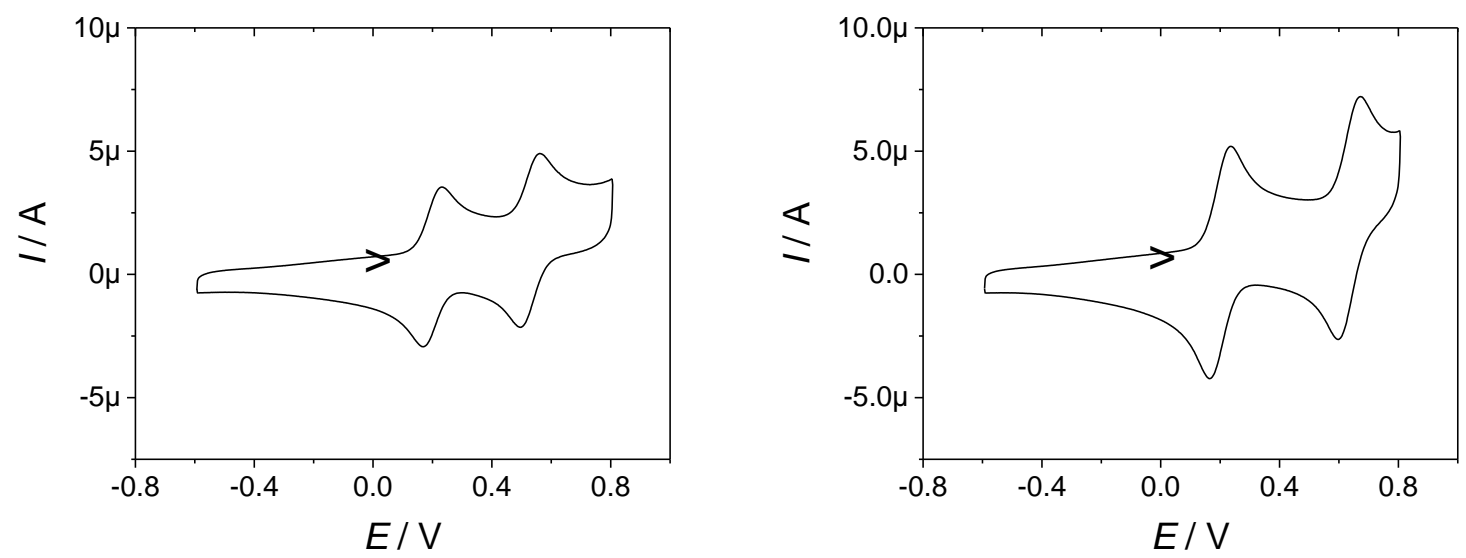

Figure S16. Cyclic voltammetry curves of complexes $\mathbf{2}$ and $\mathbf{3}$ in $\mathrm{CH}_{2} \mathrm{Cl}_{2}$ solutions containing $0.1 \mathrm{M}$ TBAP. The concentrations are 0.2 and $0.28 \mathrm{mM}$ respectively (for solubility reasons). $T=293 \mathrm{~K}$, vitreous carbon working electrode. The potentials are given versus the $\mathrm{Fc}^{+} / \mathrm{Fc}$ reference. 

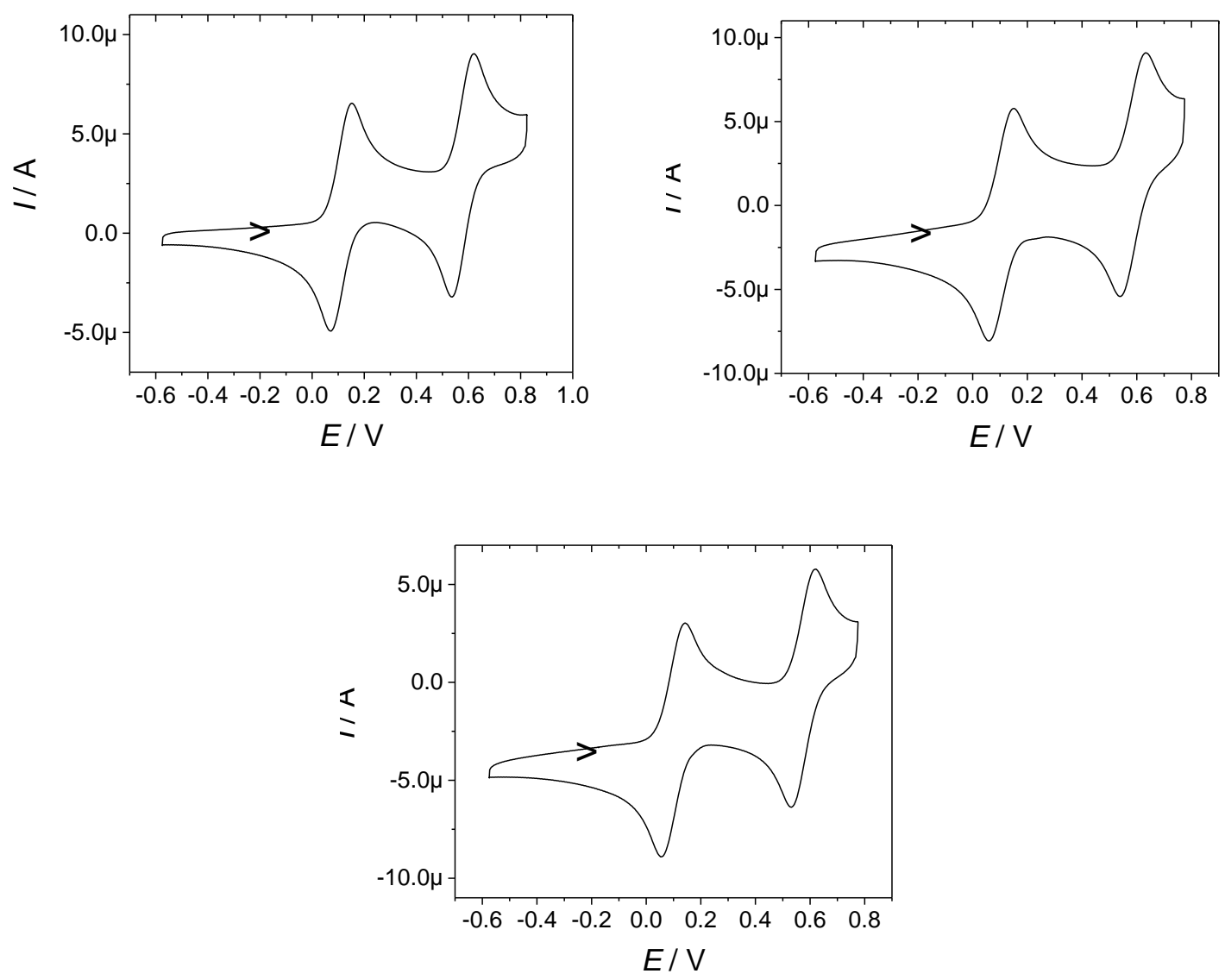

Figure S17. Cyclic voltammetry curves of complex 1 (top, left), the electrogenerated $\mathbf{1}^{+}$(top, right) and electrogenerated $\mathbf{1}^{2+}$ (bottom) in $\mathrm{CH}_{2} \mathrm{Cl}_{2}$ solutions containing $0.1 \mathrm{M}$ TBAP. The concentrations are $0.5 \mathrm{mM} . T=233 \mathrm{~K}$, vitreous carbon working electrode. Scan rate $0.1 \mathrm{~V} / \mathrm{s}$. The potentials are given versus the $\mathrm{Fc}^{+} / \mathrm{Fc}$ reference.
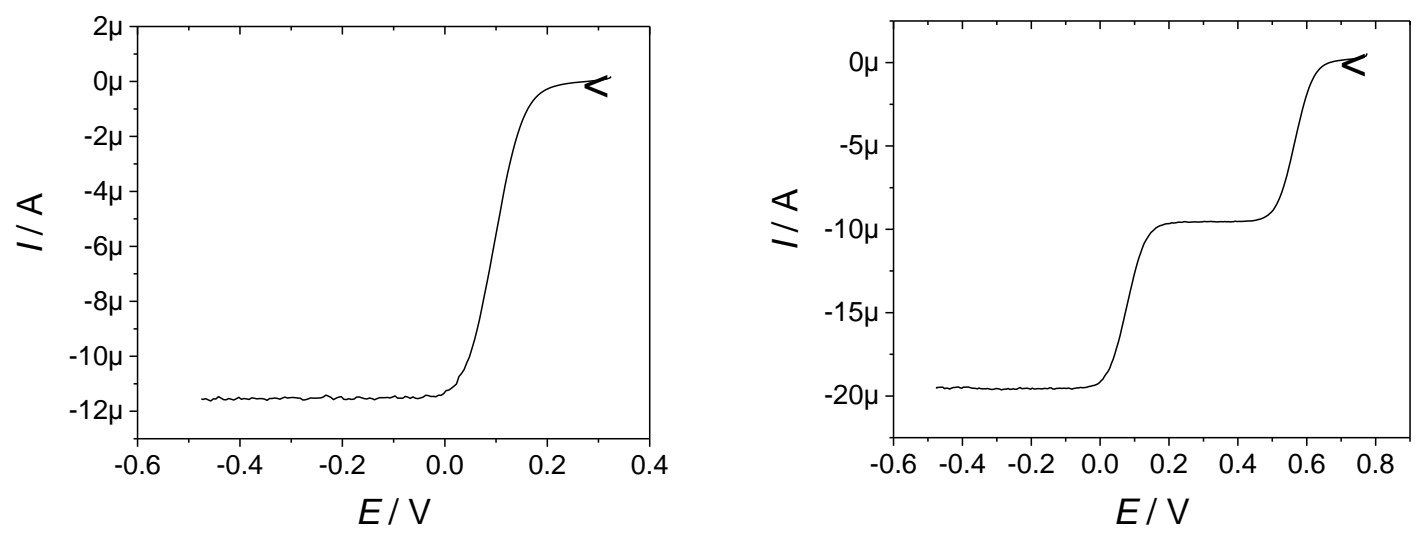

Figure S18. Rotating disc electrode voltammetry curves of the electrogenerated $\mathbf{1}^{+}$(left) and electrogenerated $\mathbf{1}^{2+}$ (right) in $\mathrm{CH}_{2} \mathrm{Cl}_{2}$ solutions containing $0.1 \mathrm{M}$ TBAP. The concentrations are 0.5 $\mathrm{mM} . T=233 \mathrm{~K}$, vitreous carbon working electrode. Scan rate $0.02 \mathrm{~V} / \mathrm{s}, 600 \mathrm{rpm}$. The potentials are given versus the $\mathrm{Fc}^{+} / \mathrm{Fc}$ reference. 

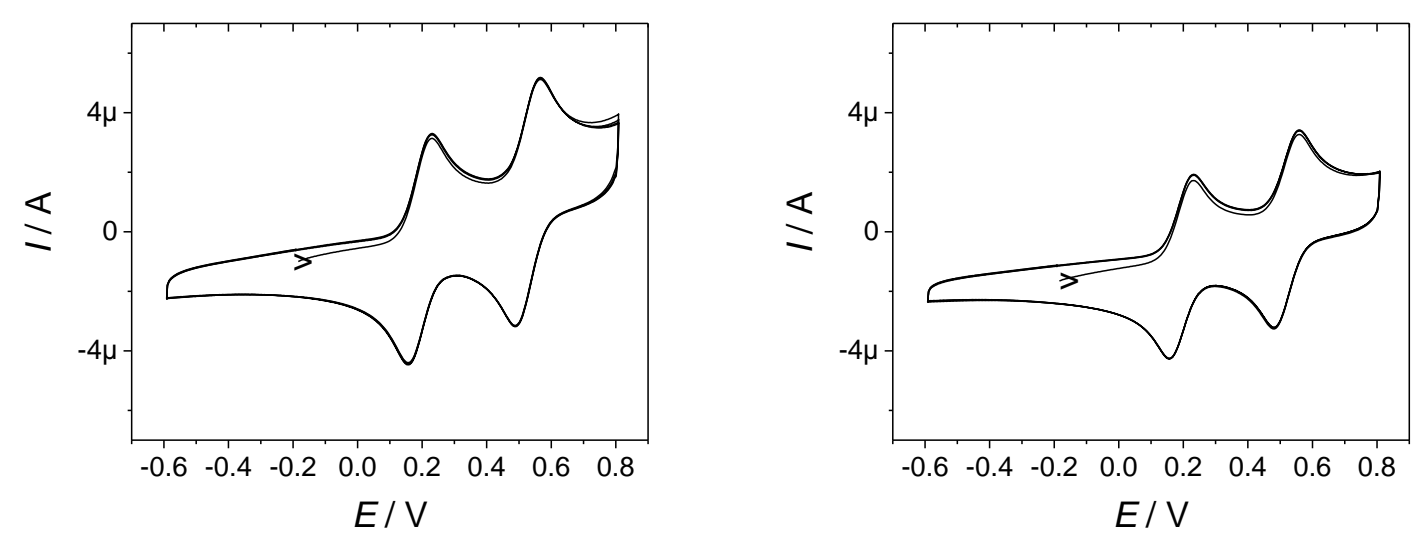

Figure S19. Cyclic voltammetry curves of the electrogenerated $\mathbf{2}^{+}$(left) and the electrogenerated $\mathbf{2}^{\mathbf{2 +}}$ (right) in $\mathrm{CH}_{2} \mathrm{Cl}_{2}$ solutions containing $0.1 \mathrm{M}$ TBAP. The concentrations are $0.28 \mathrm{mM}$. $T=233 \mathrm{~K}$, vitreous carbon working electrode. Scan rate $0.1 \mathrm{~V} / \mathrm{s}$. The potentials are given versus the $\mathrm{Fc}^{+} / \mathrm{Fc}$ reference.
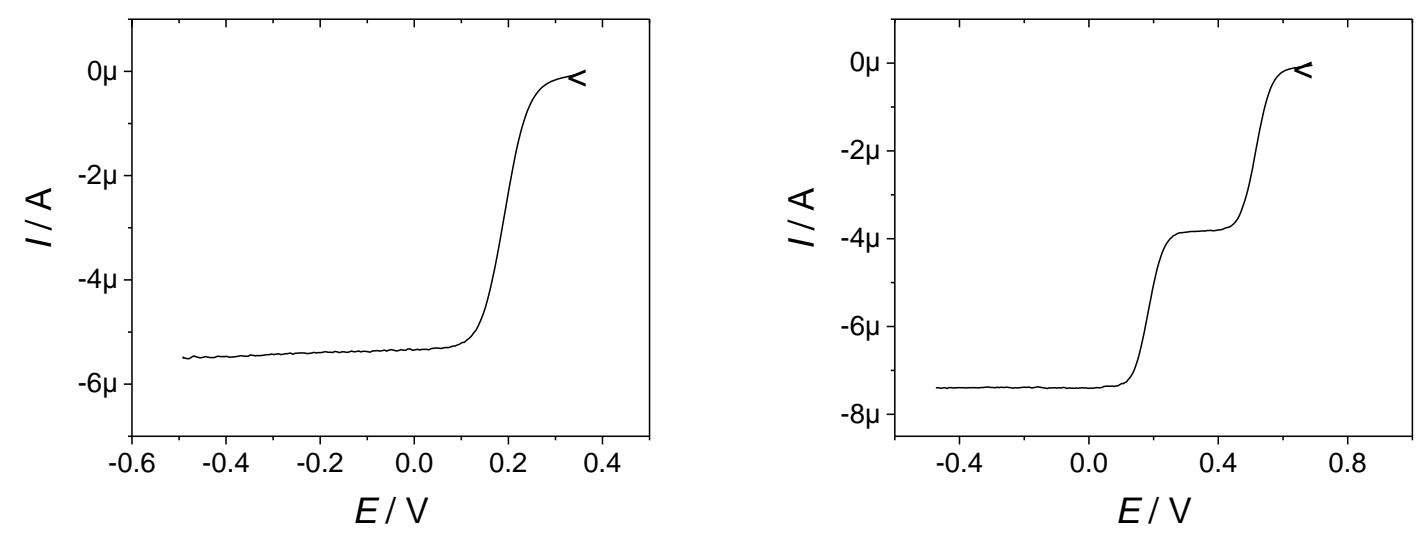

Figure S20. Rotating disc electrode voltammetry curves of the electrogenerated $\mathbf{2}^{+}$(left) and electrogenerated $\mathbf{2}^{2+}$ (right) in $\mathrm{CH}_{2} \mathrm{Cl}_{2}$ solutions containing 0.1 M TBAP. The concentrations are 0.28 $\mathrm{mM} . T=233 \mathrm{~K}$, vitreous carbon working electrode. Scan rate $0.02 \mathrm{~V} / \mathrm{s}, 600 \mathrm{rpm}$. The potentials are given versus the $\mathrm{Fc}^{+} / \mathrm{Fc}$ reference.
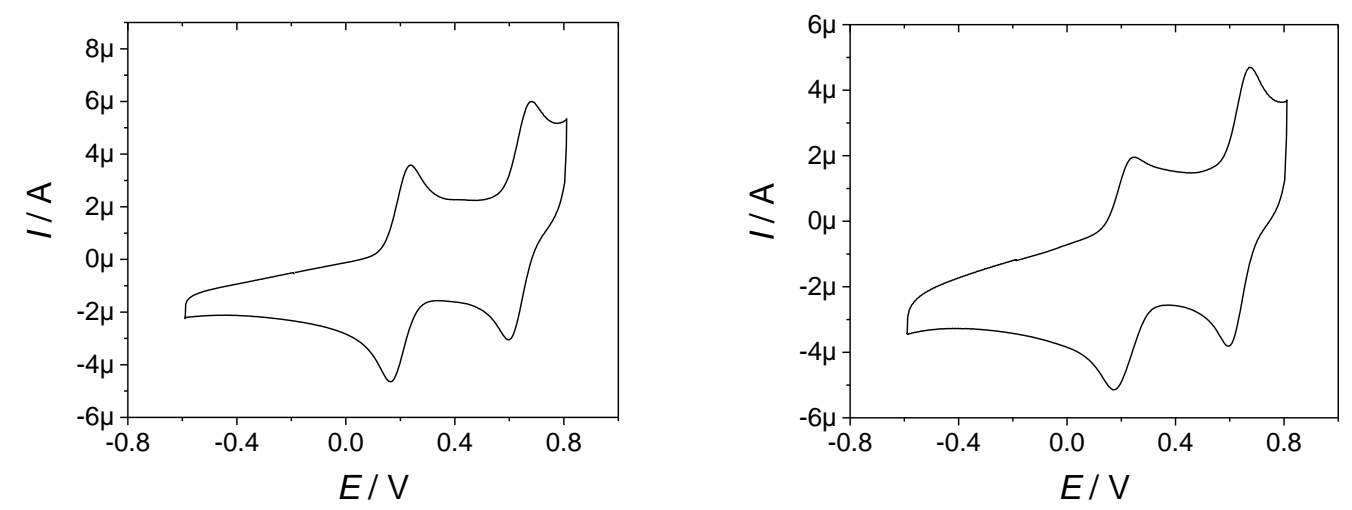

Figure S21. Cyclic voltammetry curves of the electrogenerated $\mathbf{3}^{+}$(left) and the electrogenerated $\mathbf{3}^{2+}$ (right) in $\mathrm{CH}_{2} \mathrm{Cl}_{2}$ solutions containing $0.1 \mathrm{M}$ TBAP. The concentrations are $0.28 \mathrm{mM}$. $T=233 \mathrm{~K}$, vitreous carbon working electrode. Scan rate $0.1 \mathrm{~V} / \mathrm{s}$. The potentials are given versus the $\mathrm{Fc}^{+} / \mathrm{Fc}$ reference. 

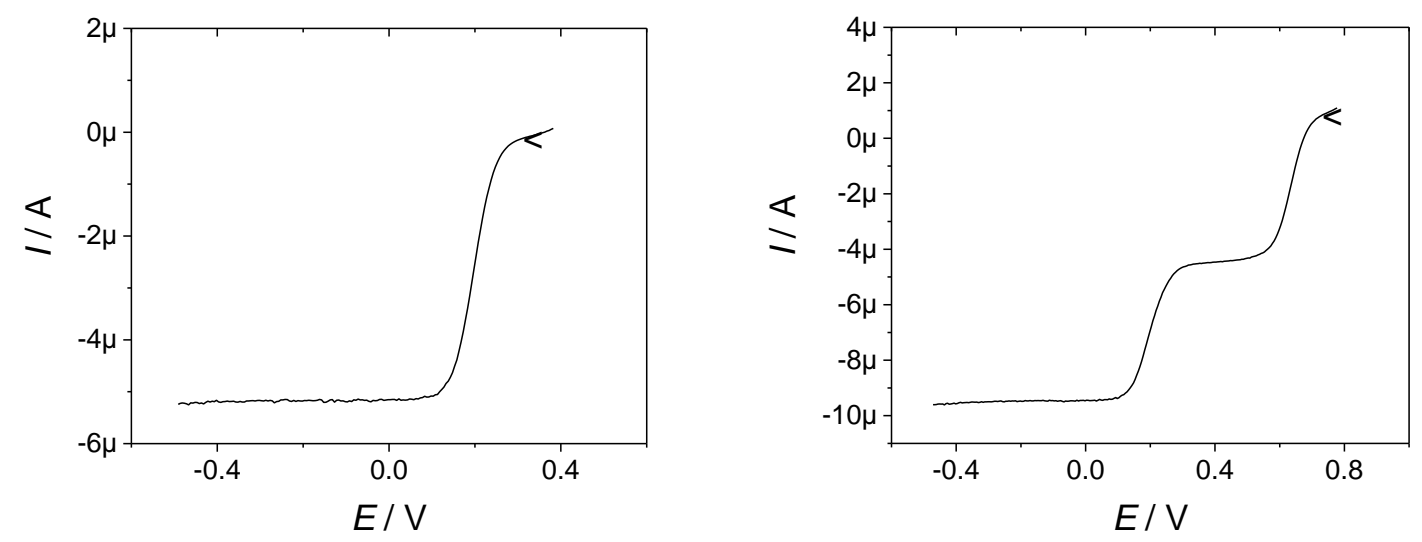

Figure S22. Rotating disc electrode voltammetry curves of the electrogenerated $\mathbf{3}^{+}$(left) and electrogenerated $\mathbf{3}^{2+}$ (right) in $\mathrm{CH}_{2} \mathrm{Cl}_{2}$ solutions containing 0.1 M TBAP. The concentrations are 0.28 $\mathrm{mM} . T=233 \mathrm{~K}$, vitreous carbon working electrode. Scan rate $0.02 \mathrm{~V} / \mathrm{s}, 600 \mathrm{rpm}$. The potentials are given versus the $\mathrm{Fc}^{+} / \mathrm{Fc}$ reference.

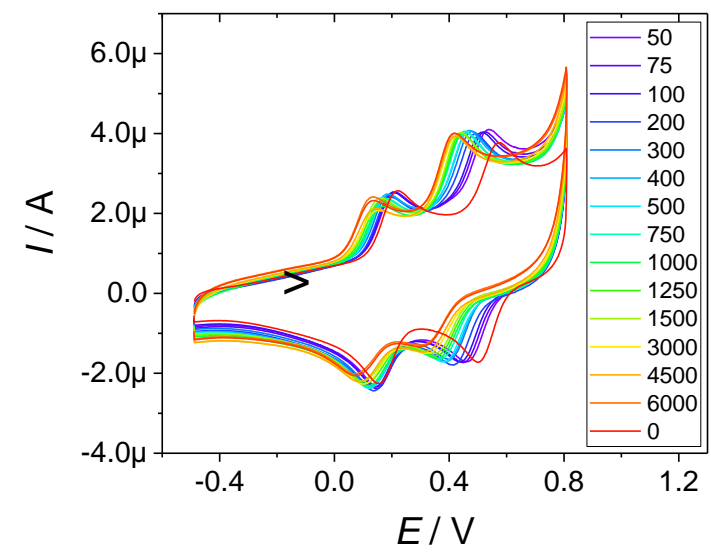

Figure S23. Cyclic voltammetry curves of 2 in $\mathrm{CH}_{2} \mathrm{Cl}_{2}$ solutions containing $0.1 \mathrm{M}$ TBAP, showing that the pyridine adduct of the cation is stable at the time-scale of the experiment. The concentration is $0.1 \mathrm{mM}$ in complex 2 . The number of molar equivalents of pyridine is indicated in the caption. $T=$ $293 \mathrm{~K}$, vitreous carbon working electrode. The potentials are given versus the $\mathrm{Fc}^{+} / \mathrm{Fc}$ reference. 


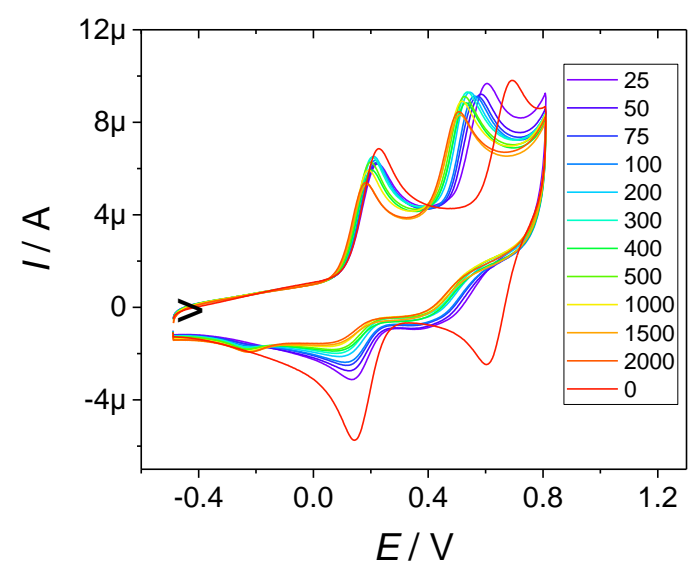

Figure S24. Cyclic voltammetry curves of 3 in $\mathrm{CH}_{2} \mathrm{Cl}_{2}$ solutions containing $0.1 \mathrm{M}$ TBAP, showing that the pyridine adduct of the cation is unstable at the time-scale of the experiment. The concentration is $0.28 \mathrm{mM}$ in complex $\mathbf{3}$. The number of molar equivalents of pyridine is indicated in the caption. $T=$ $293 \mathrm{~K}$, vitreous carbon working electrode. The potentials are given versus the $\mathrm{Fc}^{+} / \mathrm{Fc}$ reference.

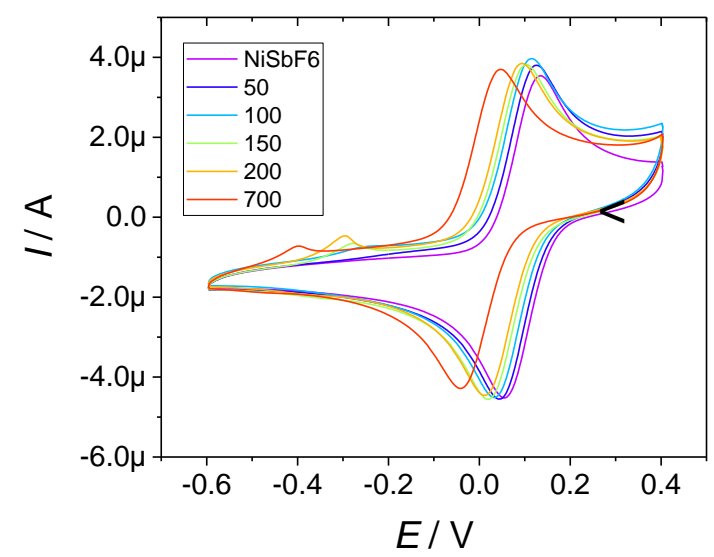

Figure S25. Cyclic voltammetry curves of $\mathbf{1}^{+} \mathrm{SbF}_{6}^{-}$in $\mathrm{CH}_{2} \mathrm{Cl}_{2}$ solutions containing $0.1 \mathrm{M}$ TBAP in the presence of increasing amounts of pyridine. The concentration is $0.5 \mathrm{mM}$ in complex $\mathbf{1}^{+} \mathrm{SbF}_{6}^{-}$. The number of molar equivalents of pyridine is indicated in the caption. $T=293 \mathrm{~K}$, vitreous carbon working electrode. The potentials are given versus the $\mathrm{Fc}^{+} / \mathrm{Fc}$ reference. 


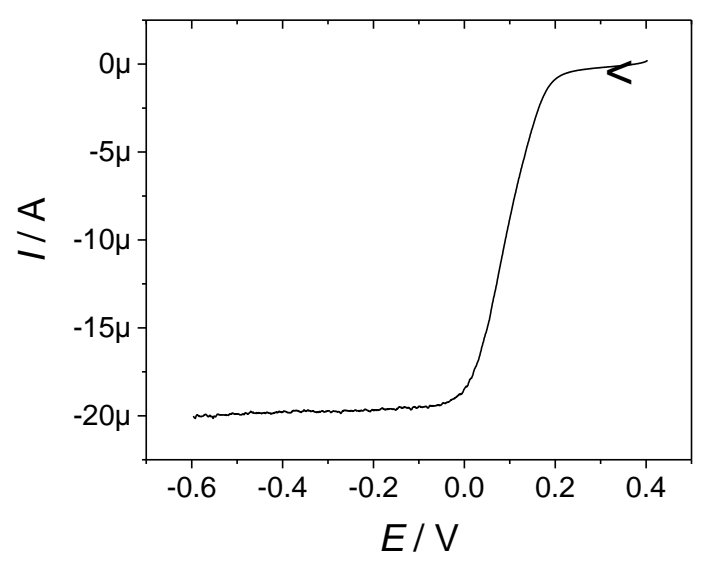

Figure S26. Rotating disc electrode voltammetry curves of the chemically generated $\mathbf{1}^{+} \mathrm{SbF}_{6}{ }^{-}$in $\mathrm{CH}_{2} \mathrm{Cl}_{2}$ solutions containing $0.1 \mathrm{M}$ TBAP. The concentration is $0.5 \mathrm{mM} . T=298 \mathrm{~K}$, vitreous carbon working electrode. Scan rate $0.02 \mathrm{~V} / \mathrm{s}, 600 \mathrm{rpm}$. The potentials are given versus the $\mathrm{Fc}^{+} / \mathrm{Fc}$ reference.

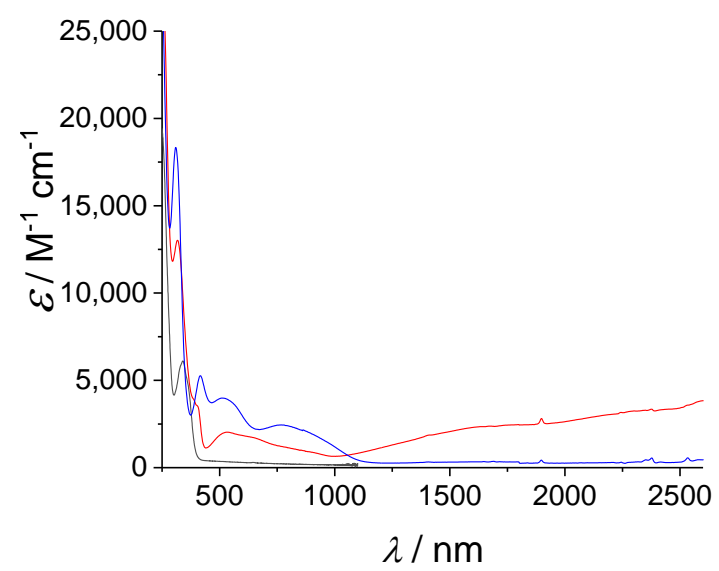

Figure S27. UV-Vis-NIR spectra of 2 (black), $\left(\mathbf{2}^{+}\right)$(red) and $\left(\mathbf{2}^{2+}\right)$ (blue) in $\mathrm{CH}_{2} \mathrm{Cl}_{2}$ solutions containing $0.1 \mathrm{M}$ TBAP. $T=293 \mathrm{~K}$.

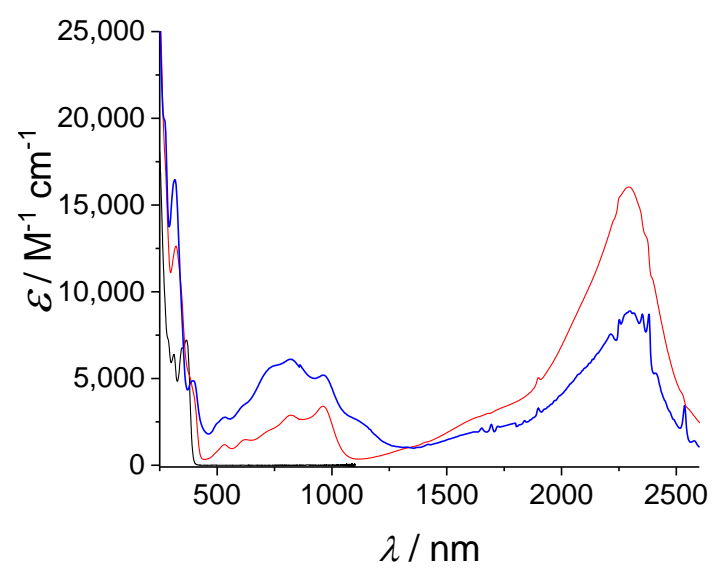

Figure S28. UV-Vis-NIR spectra of $\mathbf{3}$ (black), $\left(\mathbf{3}^{+}\right)$(red) and $\left(\mathbf{3}^{2+}\right)$ (blue) in $\mathrm{CH}_{2} \mathrm{Cl}_{2}$ solutions containing $0.1 \mathrm{M}$ TBAP. The dication decomposes significantly during the experiment (see the text). $T$ $=298 \mathrm{~K}$ (neutral and cation) and $253 \mathrm{~K}$ (dication). 


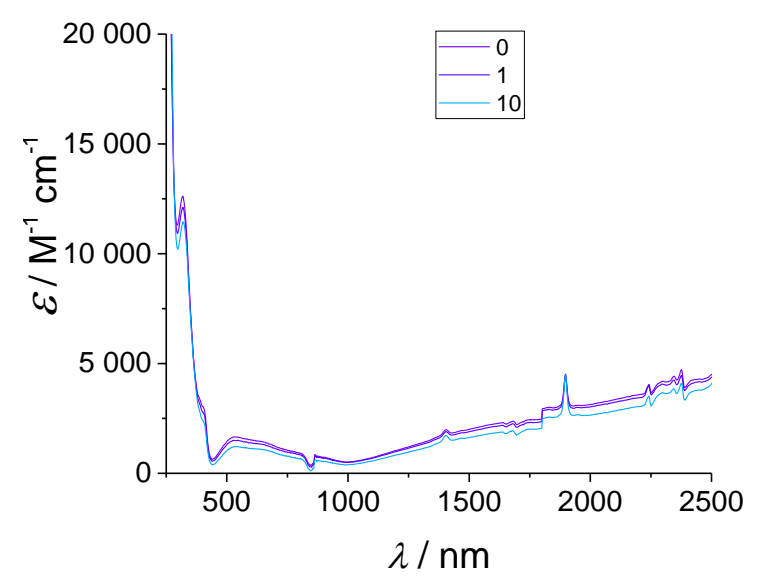

Figure S29. UV-Vis-NIR spectra of $\mathbf{2}^{+}$in $\mathrm{CH}_{2} \mathrm{Cl}_{2}$ solution containing $0.1 \mathrm{M}$ TBAP upon addition of pyridine. Concentration of $\mathbf{2}^{+}: 0.1 \mathrm{mM}$. The number of molar equivalents is indicated in the caption. $T$ $=298 \mathrm{~K} \cdot l=1.00 \mathrm{~mm}$.

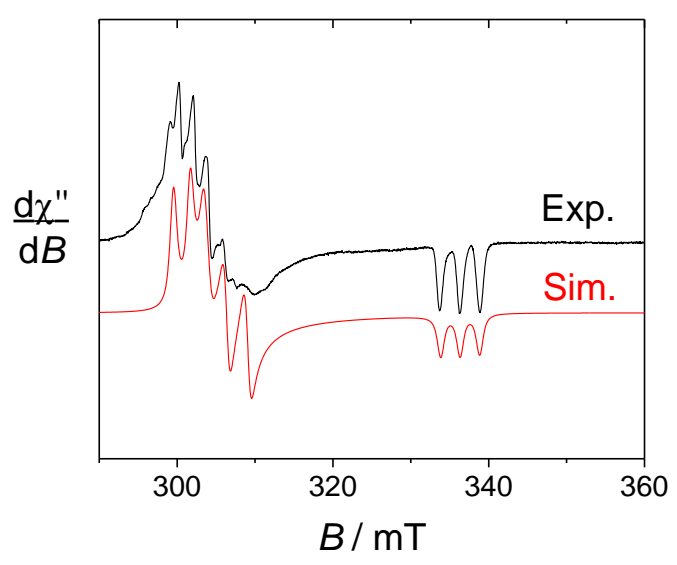

Figure S30. X-Band EPR spectrum of the electrochemically generated $\mathbf{1}_{\mathbf{P y}}{ }^{+}$in $0.5 \mathrm{mM} \mathrm{CH}_{2} \mathrm{Cl}_{2}(+0.1$ M TBAP) containing $0.5 \mathrm{mM}$ pyridine. The contribution of $\mathbf{1}^{+}$was substracted from the experimental spectrum. The black line is the experimental spectrum, the red line is a simulation by using the parameters given in Table 6. Microwave Freq. $9.43 \mathrm{GHz}$, power $1.1 \mathrm{~mW}$; Mod. Freq. $100 \mathrm{KHz}$, Amp. $0.3 \mathrm{mT} ; T=100 \mathrm{~K}$. 


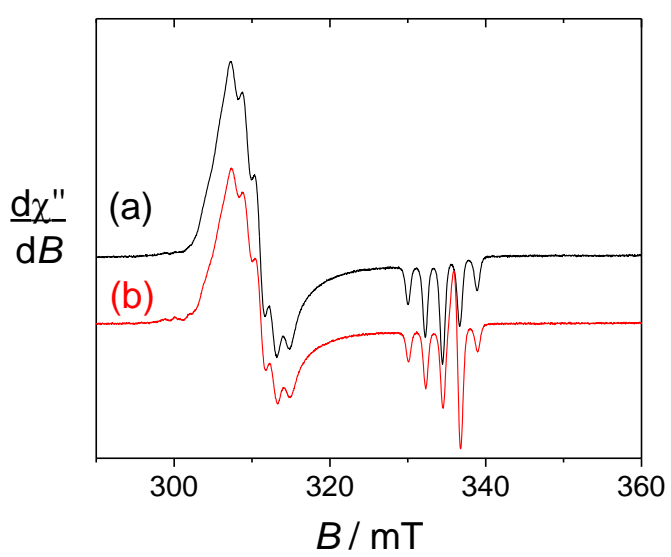

Figure S31. X-Band EPR spectra of the electrochemically generated (a) $\mathbf{1}^{+}$and (b) $\mathbf{1}^{2+}$ in $0.5 \mathrm{mM}$ $\mathrm{CH}_{2} \mathrm{Cl}_{2}$ (+0.1 M TBAP) containing 10 molar equivalents of pyridine. The addition was realized at 233 $\mathrm{K}$ and the spectrum was taken immediately after addition. Microwave Freq. $9.43 \mathrm{GHz}$, power $1.1 \mathrm{~mW}$; Mod. Freq. $100 \mathrm{KHz}$, Amp. $0.3 \mathrm{mT} ; T=100 \mathrm{~K}$.

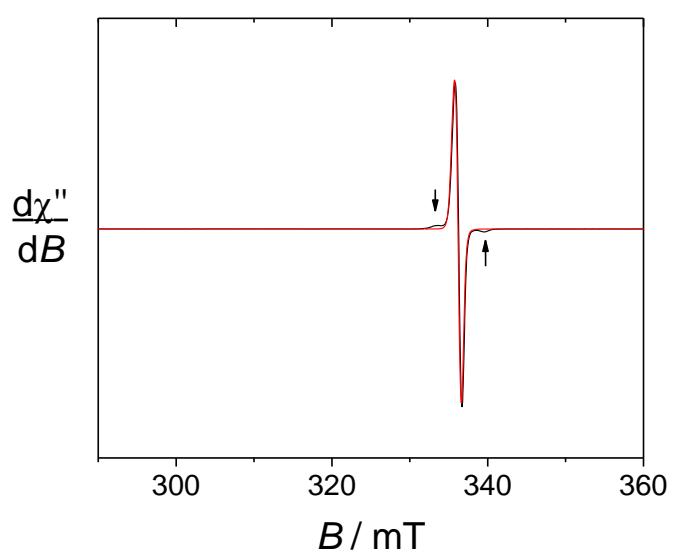

Figure S32. X-Band EPR spectrum of the electrochemically generated $\mathbf{2}^{+}$in $\mathrm{CH}_{2} \mathrm{Cl}_{2}$ solution containing 0.1 M TBAP upon addition of pyridine. Concentration of $\mathbf{2}^{+}$: $0.1 \mathrm{mM}, 1$ molar equivalent of pyridine (black) and 10 molar equivalents of pyridine (red). The spectrum at 500 molar equiv. of pyridine is identical to that in the presence of 10 molar equiv. Black arrows indicate the position of the outermost lines of $\mathbf{2}^{+}$in the absence of pyridine. Microwave Freq. $9.43 \mathrm{GHz}$, power $1.1 \mathrm{~mW}$; Mod. Freq. $100 \mathrm{KHz}$, Amp. $0.3 \mathrm{mT} ; T=100 \mathrm{~K}$. 


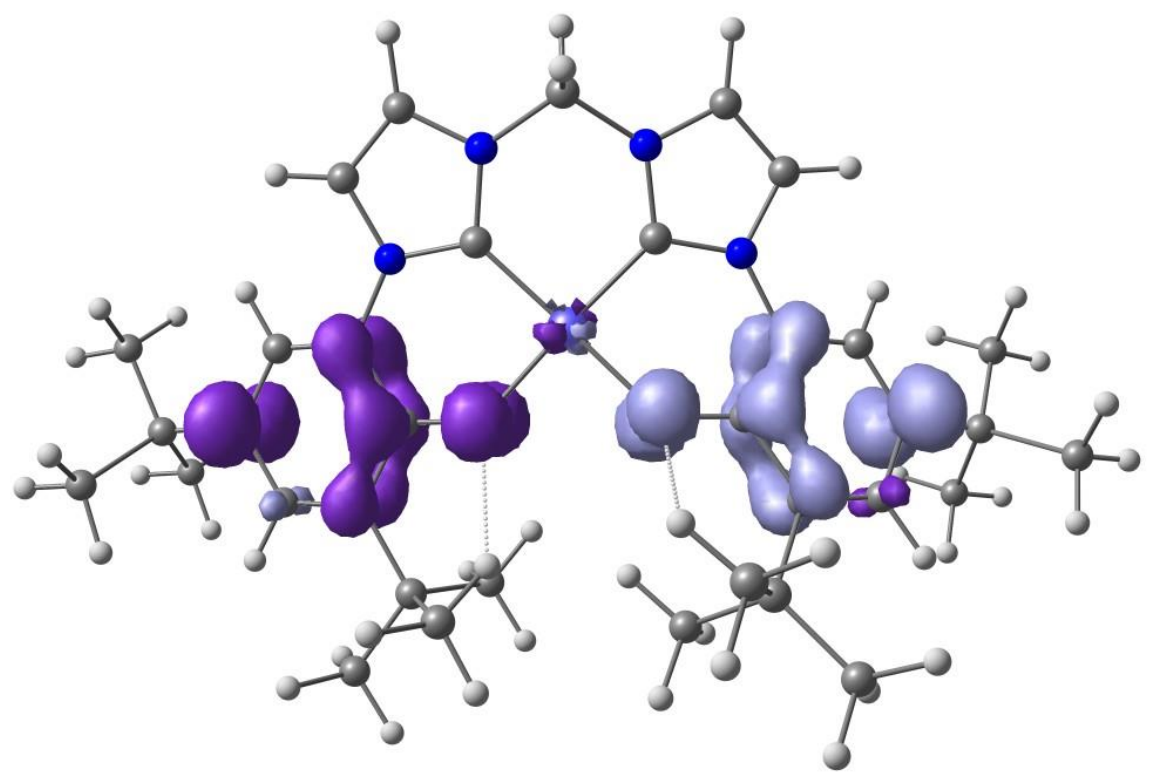

Figure S33. Spin density plot of the broken-symmetry singlet $\mathbf{1}^{2+}$ (B3LYP-D3/6-31g*).

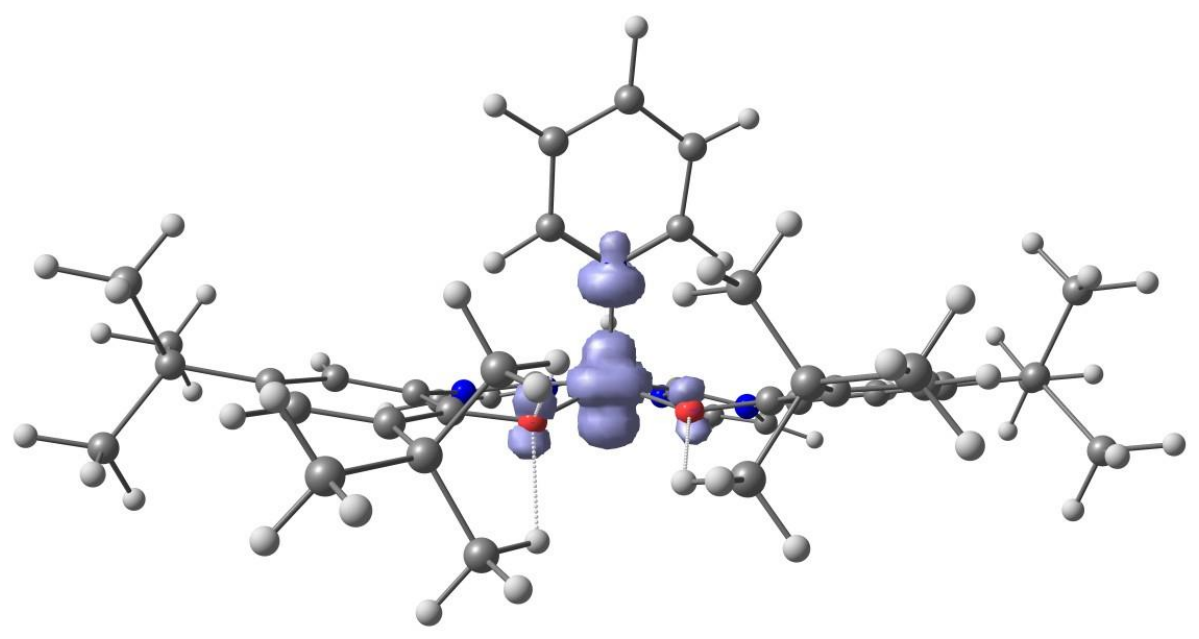

Figure S34. Spin density plot of $\mathbf{1}_{\mathbf{p y}}{ }^{+}($B3LYP-D3/6-31g*). 


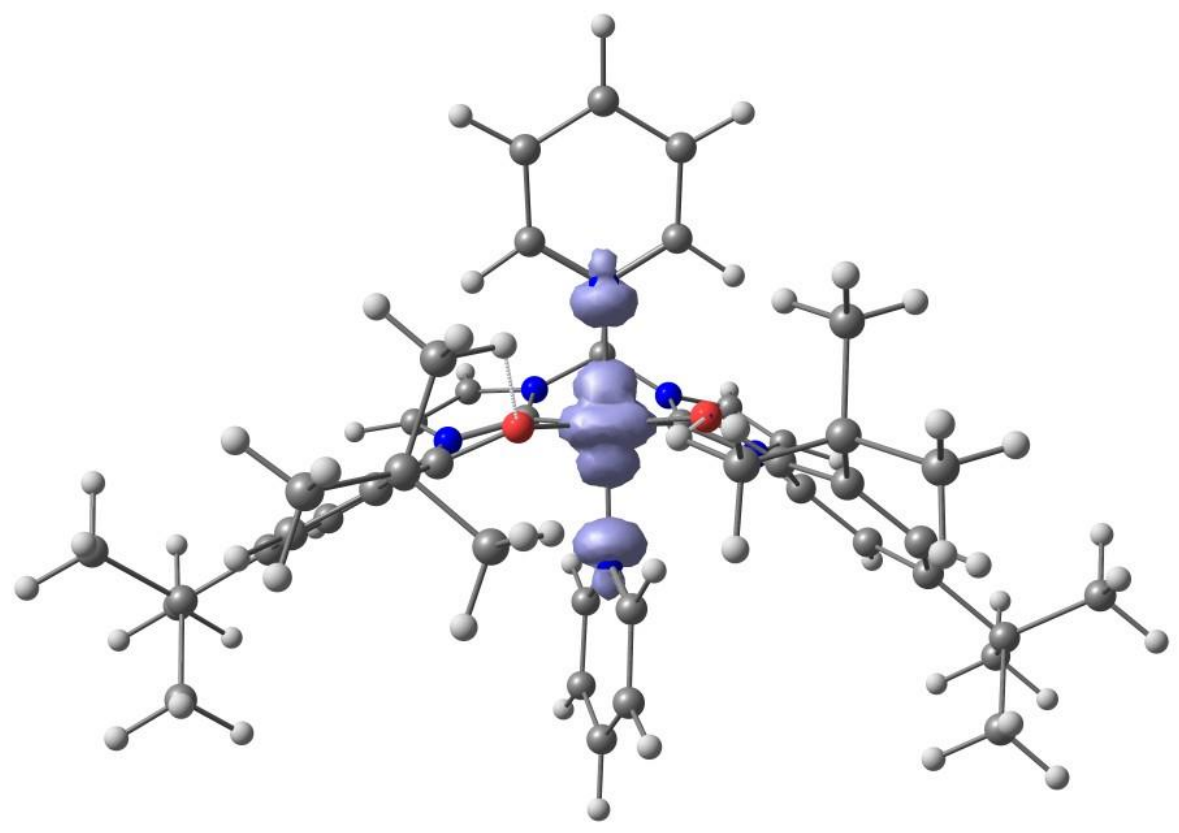

Figure S35. Spin density plot of $\mathbf{1}_{\mathrm{py} 2}{ }^{+}(\mathrm{B} 3 \mathrm{LYP}-\mathrm{D} 3 / 6-31 \mathrm{~g} *)$.

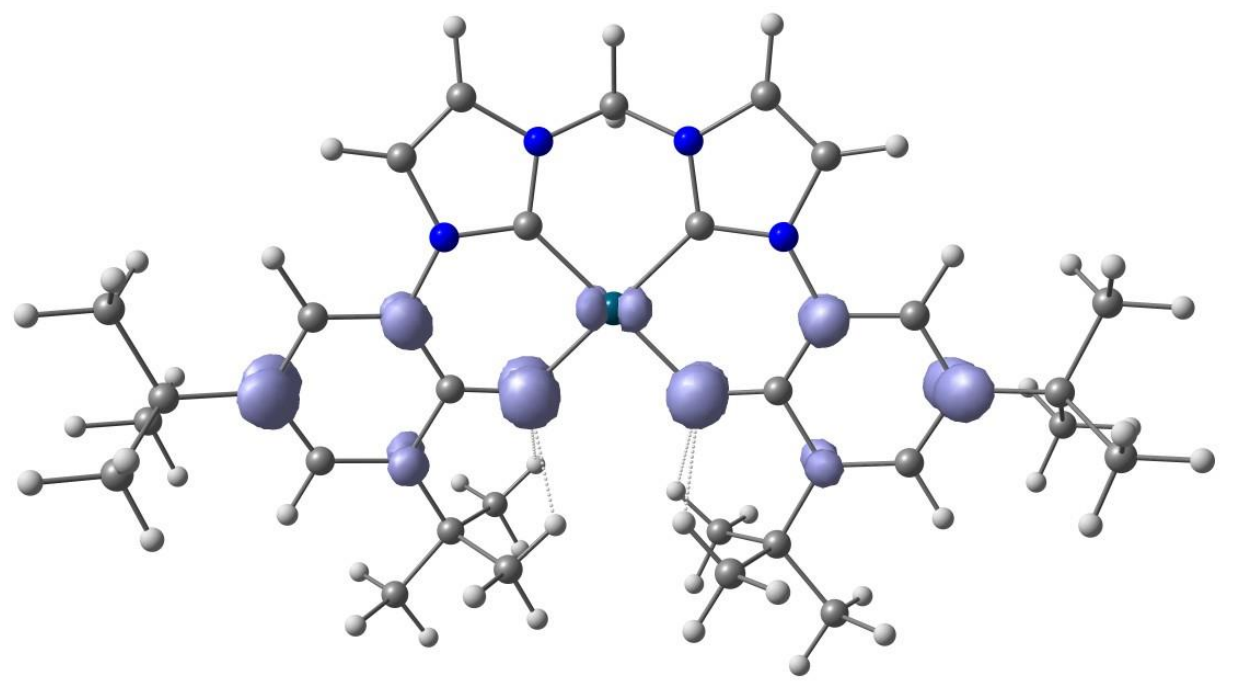

Figure S36. Spin density plot of $\mathbf{2}^{+}$(B3LYP-D3/6-31g*/LANL2DZ ECP on Pd). 


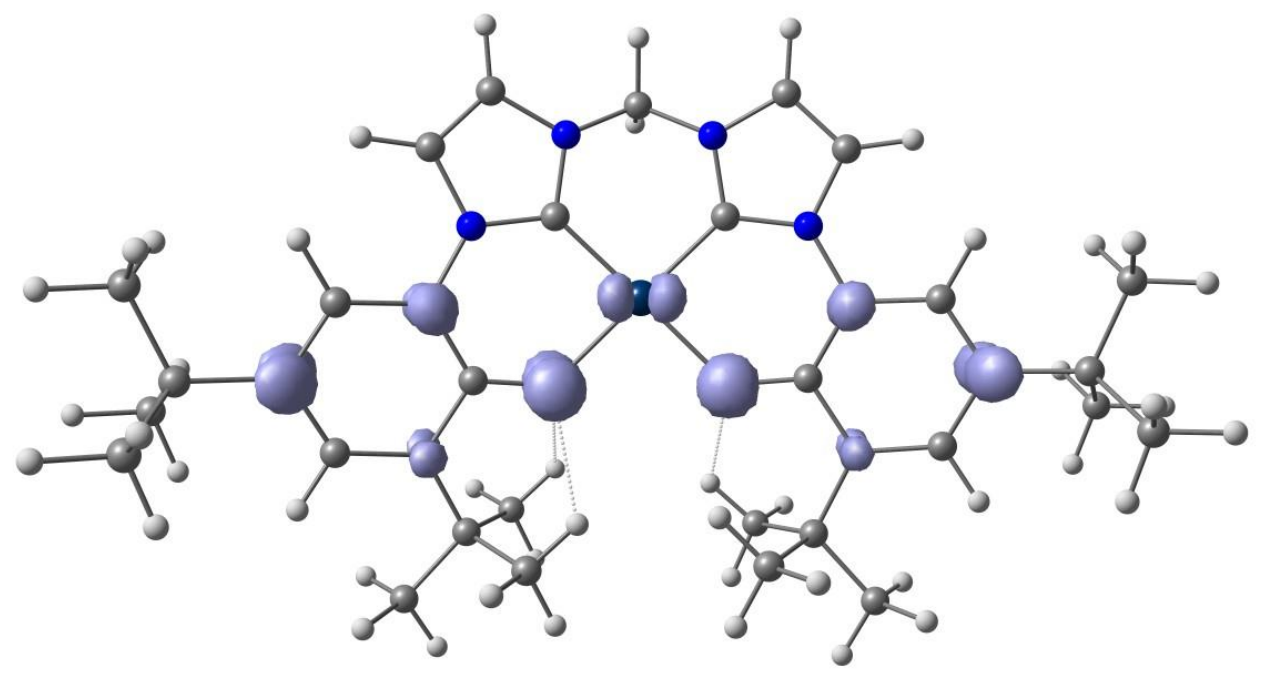

Figure S37. Spin density plot of $\mathbf{3}^{+}$(B3LYP-D3/6-31g*/LANL2DZ ECP on Pt).

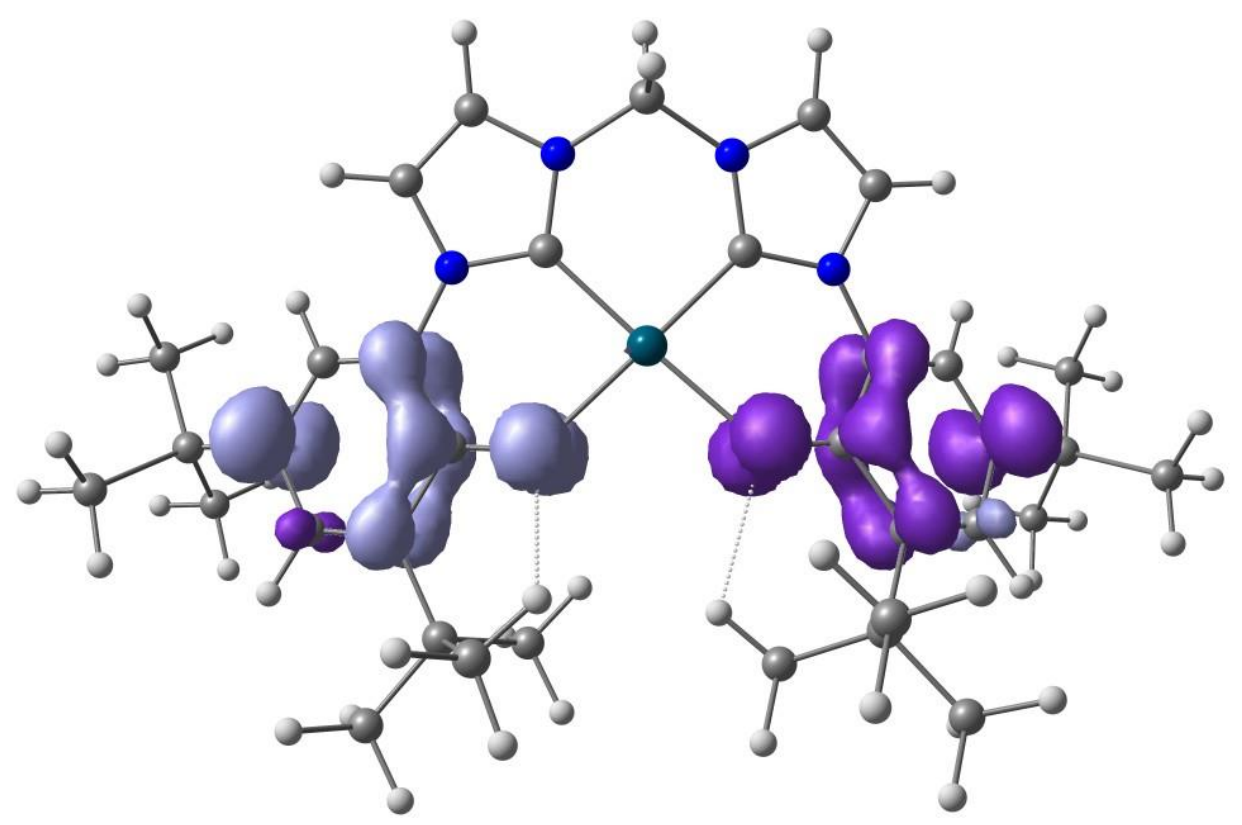

Figure S38. Spin density plot of the broken-symmetry singlet $\mathbf{2}^{2+}$ (B3LYP-D3/6-31g*/ LANL2DZ ECP on Pd). 


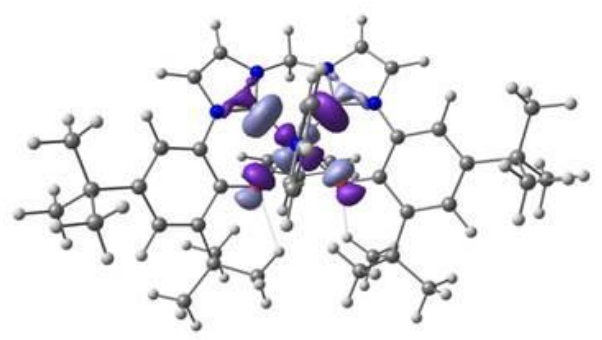

$26553 \mathrm{~cm}^{-1}\left(f_{\text {osc }}=0.0736\right) \uparrow$

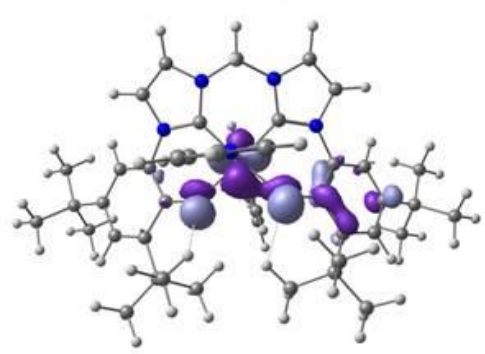

Figure S39. TD-DFT calculated electronic excitations for $\mathbf{1}_{\mathbf{p y 2}}{ }^{+}$(B3LYP-D3/TZVP/PCM). 

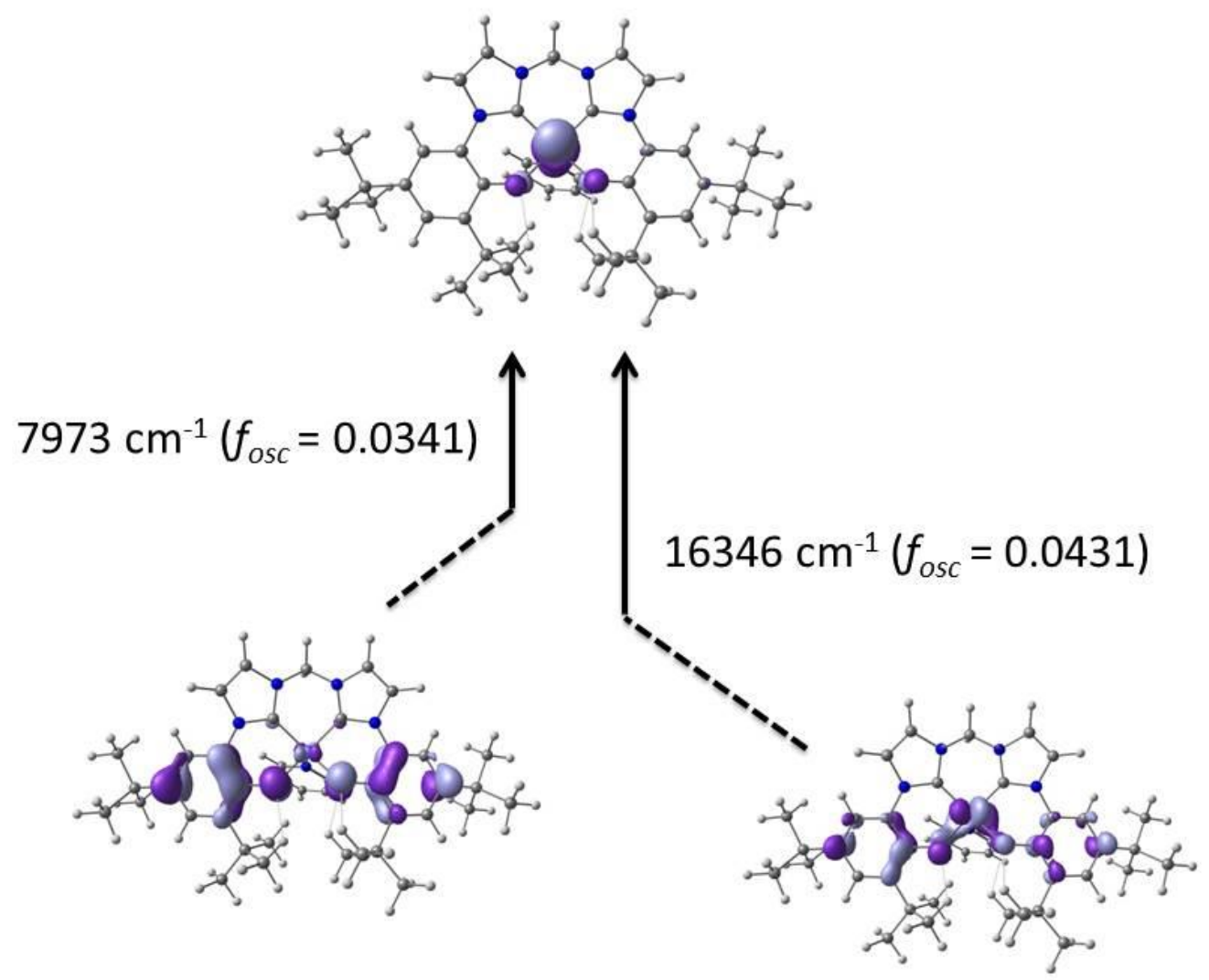

Figure S40. TD-DFT calculated electronic excitations for $\mathbf{1}_{\mathbf{p y}}{ }^{+}$(B3LYP-D3/TZVP/PCM). 

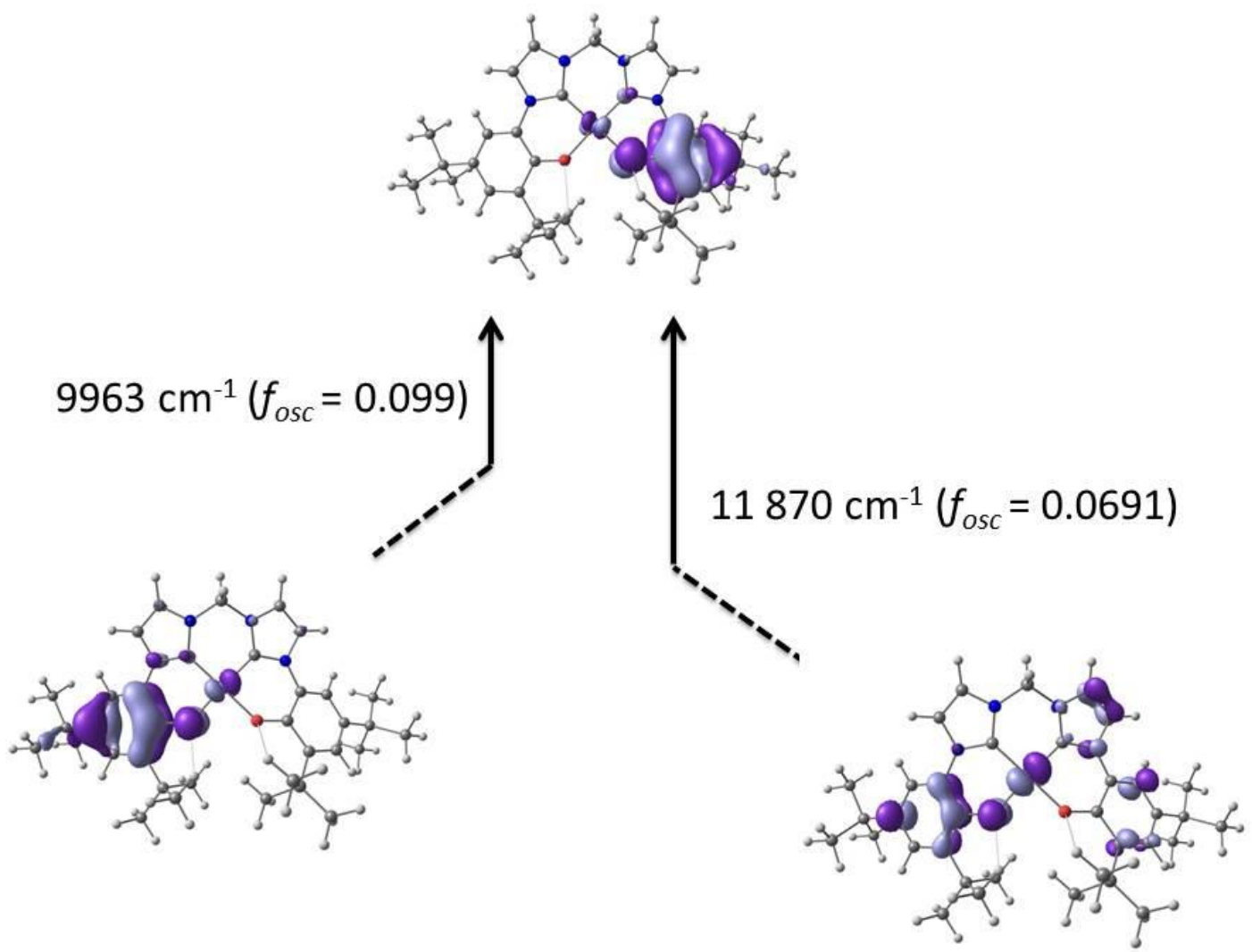

Figure S41. TD-DFT calculated electronic excitations for $\mathbf{1}^{2+}$ (B3LYP-D3/TZVP/PCM). 

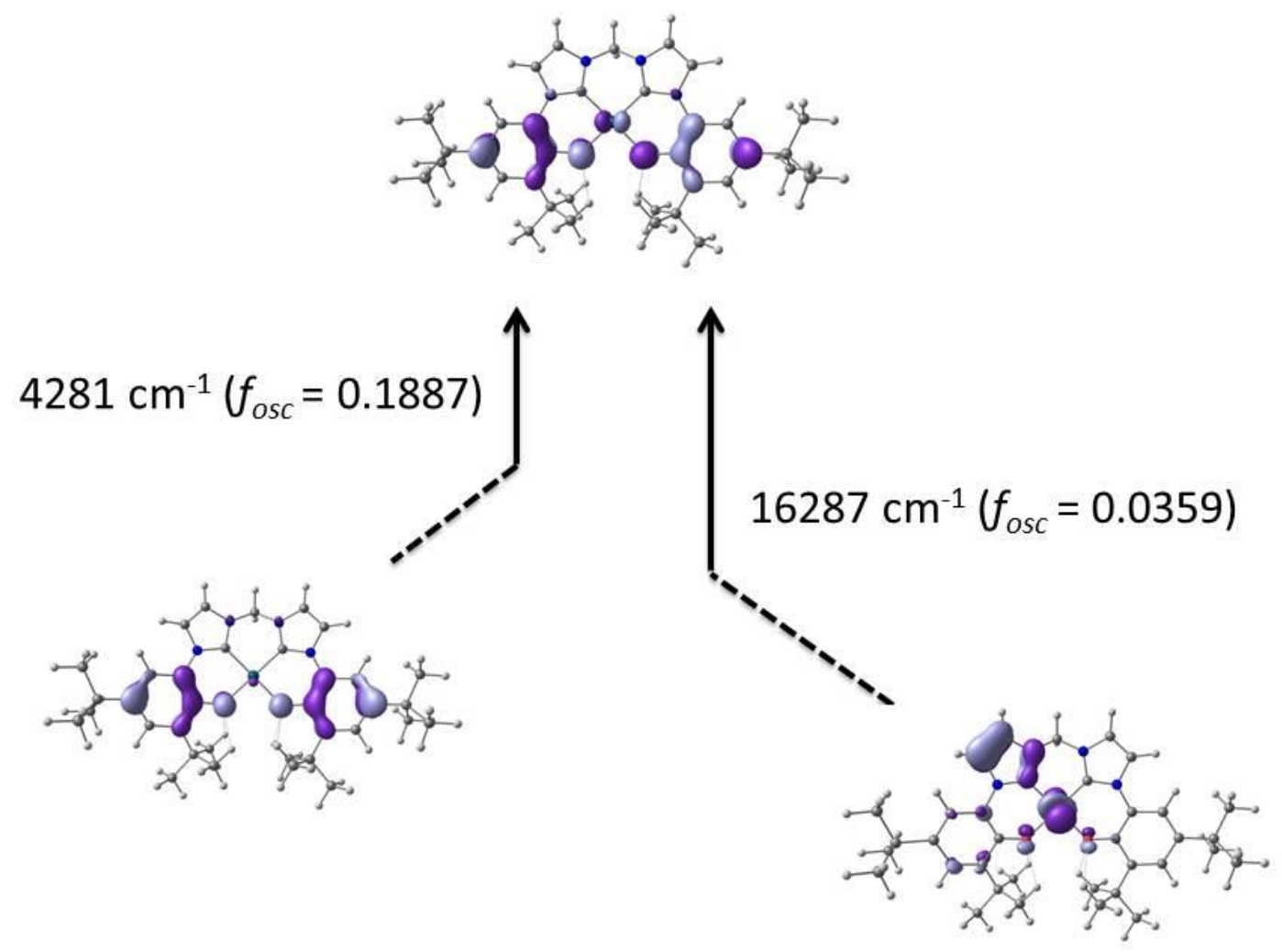

Figure S42. TD-DFT calculated electronic excitations for $\mathbf{2}^{+}$(B3LYP-D3/TZVP/PCM). 

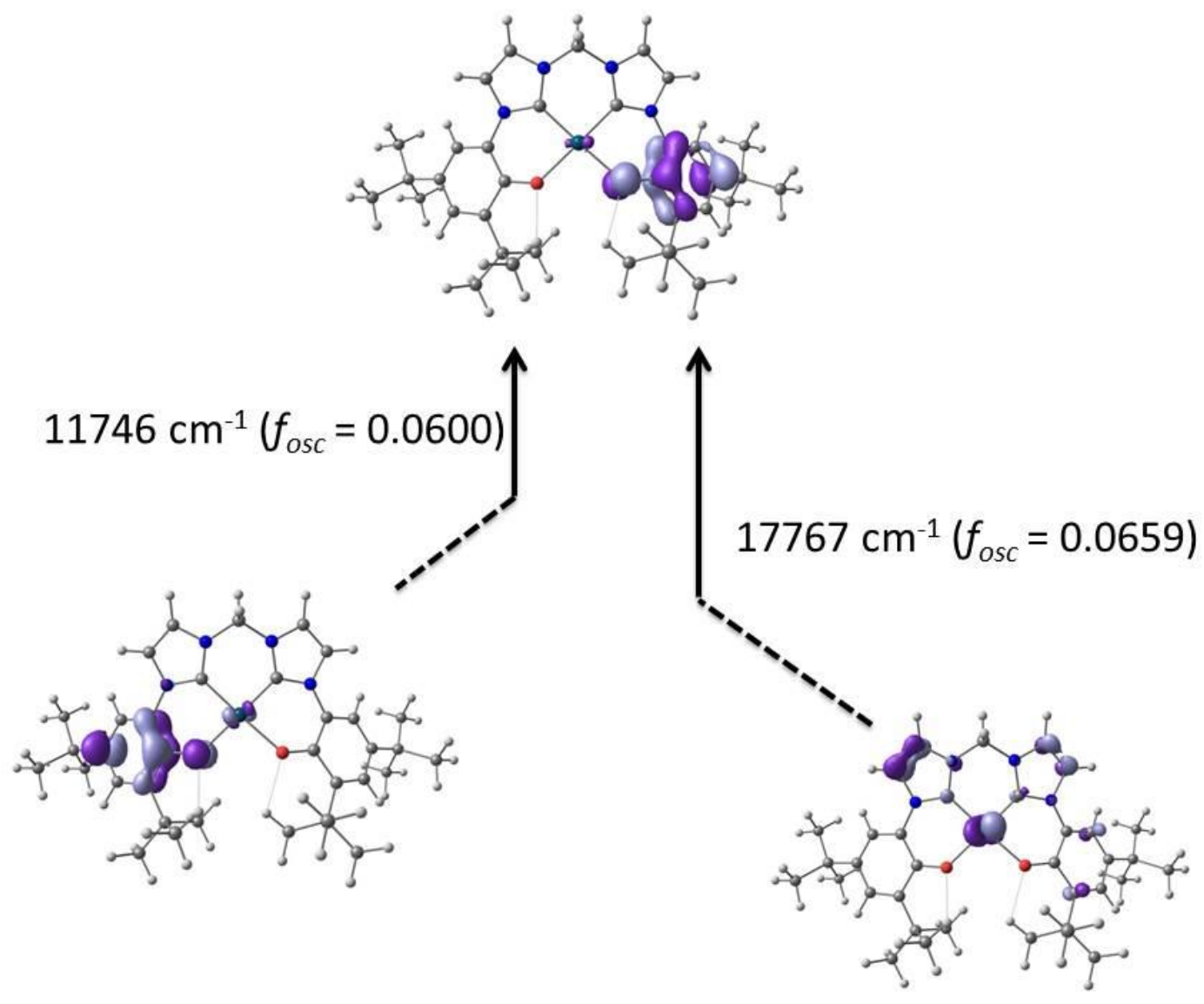

Figure S43. TD-DFT calculated electronic excitations for $\mathbf{2}^{2+}$ (B3LYP-D3/TZVP/PCM). 

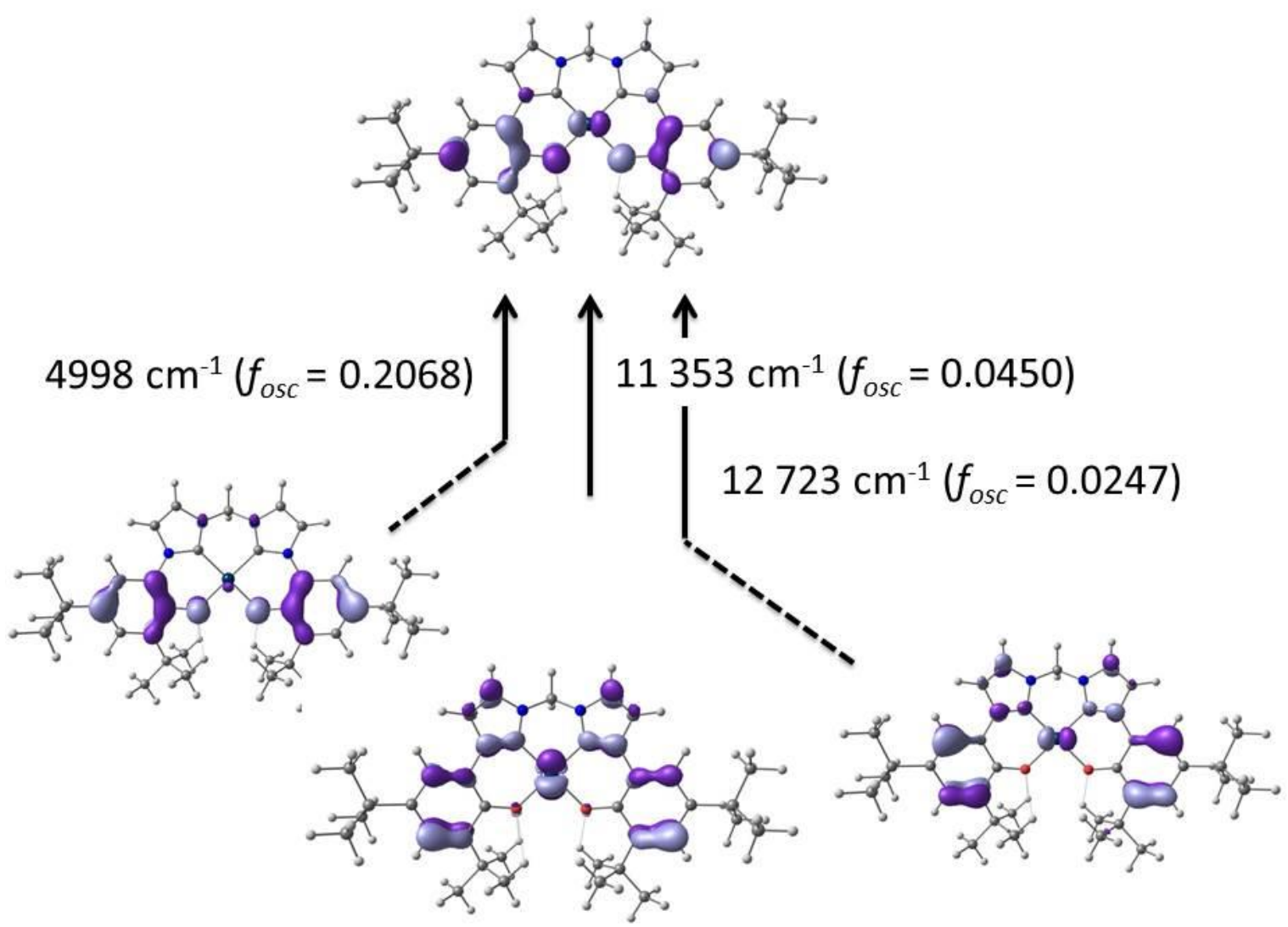

Figure S44. TD-DFT calculated electronic excitations for $3^{+}$(B3LYP-D3/TZVP/PCM). 


\section{$\underline{\text { Tables }}$}

Table S1. Bond distances within the ligand (in $\AA$ from the X-Ray crystal structures)

\begin{tabular}{|l|l|l|l|l|}
\hline Complex & $\mathbf{1}$ & $\mathbf{1}^{+}$ & $\mathbf{2}$ & $\mathbf{3}$ \\
\hline C1-O1 & $1.326(2)$ & $1.329(9)$ & $1.313(3)$ & $1.323(9)$ \\
\hline C1-C2 & $1.432(3)$ & $1.434(11)$ & $1.437(5)$ & $1.431(11)$ \\
\hline C2-C3 & $1.396(3)$ & $1.359(11)$ & $1.385(4)$ & $1.388(12)$ \\
\hline C3-C4 & $1.397(3)$ & $1.405(12)$ & $1.395(6)$ & $1.399(13)$ \\
\hline C4-C5 & $1.374(3)$ & $1.383(11)$ & $1.382(6)$ & $1.382(13)$ \\
\hline C5-C6 & $1.402(2)$ & $1.391(11)$ & $1.387(5)$ & $1.414(12)$ \\
\hline C1-C6 & $1.407(3)$ & $1.403(11)$ & $1.411(5)$ & $1.410(11)$ \\
\hline C11-O2 & - & $1.285(9)$ & & - \\
\hline C11-C12 & - & $1.446(11)$ & & - \\
\hline C12-C13 & - & $1.371(11)$ & & - \\
\hline C13-C14 & - & $1.405(12)$ & & - \\
\hline C14-C15 & - & $1.380(12)$ & & - \\
\hline C15-C16 & - & $1.365(11)$ & & - \\
\hline C11-C16 & - & $1.431(11)$ & & - \\
\hline
\end{tabular}

Table S2. Coordination bond distances (in $\AA{ }^{[a]}$

\begin{tabular}{|l|l|l|l|l|l|l|}
\hline Complex & $\mathbf{1}^{[\mathrm{b}]}$ & $\mathbf{1}^{[\mathrm{c}]}$ & $\mathbf{2}^{[\mathrm{b}]}$ & $\mathbf{2}^{[\mathrm{d}]}$ & $\mathbf{3}^{[\mathrm{b}]}$ & $\mathbf{3}^{[\mathrm{d}]}$ \\
\hline M-O1 & 1.868 & 1.881 & 2.061 & 2.021 & 2.058 & 2.028 \\
\hline M-O2 & 1.878 & 1.881 & 2.049 & 2.021 & 2.051 & 2.028 \\
\hline M-C7 & 1.844 & 1.843 & 1.948 & 1.927 & 1.935 & 1.918 \\
\hline M-C17 & 1.843 & 1.843 & 1.951 & 1.927 & 1.936 & 1.918 \\
\hline
\end{tabular}

${ }^{[a]}$ Numbering used:<smiles>[CH]1NC2CCCCC2OC12CNC1CCCCC1O2</smiles>

${ }^{[b]}$ From the X-Ray crystal structures

${ }^{[c]}$ from a B3LYP-D3 calculation with 6-31 $\mathrm{g}^{*}$ basis set on all atoms.

${ }^{\text {[d] }}$ from a B3LYP-D3 calculation with 6-31 $\mathrm{g} *$ basis set on the C, H, N, O and LanL2DZ ECP on the metal center.

Table S3. Mulliken spin populations in the cations ${ }^{[a]}$

\begin{tabular}{|l|l|l|l|l|l|}
\hline $\begin{array}{l}\text { Functional } \\
\text { Complex }\end{array}$ & $\begin{array}{l}\text { B3LYP } \\
\mathbf{1}^{+}\end{array}$ & $\begin{array}{l}\text { CAM- } \\
\text { B3LYP }^{[b]} \mathbf{1}^{+}\end{array}$ & $\begin{array}{l}\text { B3LYP-D3 } \\
\mathbf{1}^{+}\end{array}$ & $\begin{array}{l}\text { B3LYP-D3 } \\
\mathbf{2}^{+}\end{array}$ & $\begin{array}{l}\text { B3LYP-D3 } \\
\mathbf{3}^{+}\end{array}$ \\
\hline Metal & 0.17 & 0.10 & 0.07 & 0.04 & 0.07 \\
\hline O1 & 0.16 & 0.29 & 0.17 & 0.18 & 0.18 \\
\hline O2 & 0.17 & 0.06 & 0.17 & 0.18 & 0.18 \\
\hline C2 & 0.08 & 0.19 & 0.09 & 0.09 & 0.08 \\
\hline C4 & 0.14 & 0.32 & 0.16 & 0.16 & 0.15 \\
\hline
\end{tabular}




\begin{tabular}{|l|l|l|l|l|l|}
\hline C6 & 0.11 & 0.24 & 0.11 & 0.11 & 0.11 \\
\hline C12 & 0.08 & 0.02 & 0.09 & 0.10 & 0.08 \\
\hline C14 & 0.15 & 0.03 & 0.17 & 0.17 & 0.16 \\
\hline C16 & 0.12 & 0.04 & 0.12 & 0.12 & 0.12 \\
\hline
\end{tabular}

${ }^{[a]}$ Numbering used:<smiles></smiles>

${ }^{[\mathrm{b}]} 6-31 \mathrm{~g} *$ basis set on all atoms.

${ }^{[c]}$ 6-31 $\mathrm{g}^{*}$ basis set on the $\mathrm{C}, \mathrm{H}, \mathrm{N}, \mathrm{O}$ and LanL2DZ ECP on the metal center.

Table S4. Mulliken spin populations in the dications ${ }^{[a]}$

\begin{tabular}{|l|l|l|}
\hline $\begin{array}{l}\text { Functional } \\
\text { Complex }\end{array}$ & $\begin{array}{l}\text { B3LYP-D3 }^{[\mathrm{b}]} \\
\mathbf{1}^{2+}\end{array}$ & $\begin{array}{l}\text { B3LYP-D3 }^{[\mathrm{c}]} \\
\mathbf{3}^{2+}\end{array}$ \\
\hline Metal & 0.00 & 0.00 \\
\hline O1 & -0.24 & -0.25 \\
\hline O2 & 0.24 & 0.25 \\
\hline C2 & -0.16 & -0.17 \\
\hline C4 & -0.32 & -0.33 \\
\hline C6 & -0.17 & -0.17 \\
\hline C12 & 0.16 & 0.17 \\
\hline C14 & 0.33 & 0.34 \\
\hline C16 & 0.18 & 0.17 \\
\hline
\end{tabular}

${ }^{[a]}$ Numbering used:<smiles>C1CCC2OC3(CNC2C1)CNC1CCCCC1O3</smiles>

${ }^{[b]} 6-31 \mathrm{~g}^{*}$ basis set on all atoms.

${ }^{[c]}$ 6-31 $\mathrm{g}^{*}$ basis set on the $\mathrm{C}, \mathrm{H}, \mathrm{N}, \mathrm{O}$ and LanL2DZ ECP on the metal center. 
Table S5. Calculated energeries (in Ha) and magnetic interaction in the dications

\begin{tabular}{|l|l|l|l|l|l|}
\hline Complex & E(SCF) Triplet & E(SCF) BS & $\langle\mathrm{S}\rangle^{2}$ Triplet & $\langle\mathrm{S}\rangle^{2}$ Singlet & $J^{[\mathrm{c}]}$ \\
\hline $\mathbf{1}^{2+[\mathrm{a}]}$ & -3238.71502838 & -3238.71605822 & 2.0263 & 0.9760 & -215 \\
\hline $\mathbf{2}^{2+[\mathbf{b}]}$ & -1857.23895062 & -1857.23942516 & 2.0290 & 1.0100 & -102 \\
\hline $\mathbf{3}^{2+[\mathbf{b}]}$ & -1849.69067015 & -1849.69172851 & 2.0265 & 0.9814 & -223 \\
\hline
\end{tabular}

${ }^{[a]}$ B3LYP-D3/6-31g*

${ }^{[b]}$ B3LYP-D3/6-31g*/LanL2DZ ECP for Pd and Pt

${ }^{\text {[c] }}$ Calculated according to the Yamaguchi formula (given in $\mathrm{cm}^{-1}$ ):

$J=-\left(\mathrm{E}_{\text {triplet }}-\mathrm{E}_{\mathrm{BS}}\right) /\left(\langle\mathrm{S}\rangle^{2}{ }_{\text {triplet }}-\langle\mathrm{S}\rangle_{\mathrm{BS}}{ }_{\mathrm{BS}}\right)($ where $\mathrm{BS}=$ broken symmetry $)$ 


\section{XYZ coordinates}

1

\begin{tabular}{|c|c|c|c|}
\hline $\mathrm{Ni}$ & -0.011983000 & 1.029963000 & 0.345533000 \\
\hline 0 & -1.258341000 & -0.340032000 & 0.098597000 \\
\hline 0 & 1.299928000 & -0.298808000 & 0.544668000 \\
\hline $\mathrm{N}$ & -2.678971000 & 2.101015000 & -0.051081000 \\
\hline $\mathrm{N}$ & 2.642714000 & 2.131543000 & 0.034990000 \\
\hline $\mathrm{N}$ & 1.166922000 & 3.667545000 & 0.391628000 \\
\hline $\mathrm{N}$ & -1.213451000 & 3.649910000 & 0.307367000 \\
\hline C & 2.687556000 & -2.818007000 & 0.866797000 \\
\hline $\mathrm{C}$ & 1.313477000 & 2.309143000 & 0.294516000 \\
\hline C & -2.563491000 & -0.347870000 & -0.038800000 \\
\hline C & -1.358742000 & 2.286204000 & 0.250343000 \\
\hline C & -3.276630000 & -1.596875000 & -0.116613000 \\
\hline C & -5.426151000 & -0.393472000 & -0.325826000 \\
\hline C & -3.340318000 & 0.834784000 & -0.134097000 \\
\hline C & 4.644429000 & 0.833840000 & -0.479706000 \\
\hline $\mathrm{H}$ & 5.116467000 & 1.741494000 & -0.831487000 \\
\hline C & -2.507205000 & -2.928314000 & -0.093055000 \\
\hline C & -4.736971000 & 0.806100000 & -0.263036000 \\
\hline $\mathrm{H}$ & -5.290643000 & 1.733828000 & -0.294190000 \\
\hline $\mathrm{C}$ & 3.353351000 & -1.516888000 & 0.386932000 \\
\hline C & 5.364981000 & -0.349481000 & -0.459025000 \\
\hline $\mathrm{C}$ & 6.948703000 & -1.466871000 & -2.066220000 \\
\hline $\mathrm{H}$ & 6.598793000 & -2.461257000 & -1.770576000 \\
\hline $\mathrm{H}$ & 7.992343000 & -1.562127000 & -2.391777000 \\
\hline $\mathrm{H}$ & 6.348943000 & -1.144428000 & -2.924952000 \\
\hline C & 2.044591000 & -2.606668000 & 2.258455000 \\
\hline $\mathrm{H}$ & 2.811548000 & -2.349793000 & 2.999923000 \\
\hline $\mathrm{H}$ & 1.550069000 & -3.529069000 & 2.587681000 \\
\hline $\mathrm{H}$ & 1.303458000 & -1.807542000 & 2.232195000 \\
\hline C & 2.588119000 & -0.301005000 & 0.292910000 \\
\hline C & -1.613730000 & -2.994449000 & -1.354908000 \\
\hline $\mathrm{H}$ & -2.238137000 & -3.004550000 & -2.256854000 \\
\hline $\mathrm{H}$ & -1.007037000 & -3.907582000 & -1.349609000 \\
\hline $\mathrm{H}$ & -0.944190000 & -2.135421000 & -1.407824000 \\
\hline C & 4.689240000 & -1.493938000 & 0.009425000 \\
\hline $\mathrm{H}$ & 5.250741000 & -2.417804000 & 0.064134000 \\
\hline $\mathrm{C}$ & 1.611779000 & -3.229607000 & -0.159649000 \\
\hline $\mathrm{H}$ & 0.866223000 & -2.444725000 & -0.264418000 \\
\hline $\mathrm{H}$ & 1.107774000 & -4.149295000 & 0.161517000 \\
\hline $\mathrm{H}$ & 2.066693000 & -3.415638000 & -1.140464000 \\
\hline C & 3.297176000 & 3.363037000 & -0.004469000 \\
\hline $\mathrm{H}$ & 4.355867000 & 3.455951000 & -0.166886000 \\
\hline C & 3.689608000 & -3.982195000 & 0.994634000 \\
\hline $\mathrm{H}$ & 4.140538000 & -4.248075000 & 0.031298000 \\
\hline $\mathrm{H}$ & 3.161763000 & -4.869381000 & 1.362508000 \\
\hline $\mathrm{H}$ & 4.495566000 & -3.757920000 & 1.703910000 \\
\hline $\mathrm{C}$ & 6.831348000 & -0.451880000 & -0.905038000 \\
\hline $\mathrm{C}$ & -2.405891000 & 4.299624000 & 0.051605000 \\
\hline $\mathrm{H}$ & -2.496225000 & 5.374983000 & 0.048748000 \\
\hline $\mathrm{C}$ & -6.953893000 & -0.481287000 & -0.460819000 \\
\hline C & -3.321690000 & 3.332712000 & -0.185743000 \\
\hline $\mathrm{H}$ & -4.355256000 & 3.426952000 & -0.464739000 \\
\hline $\mathrm{C}$ & 3.303697000 & 0.867948000 & -0.072542000 \\
\hline C & -4.656909000 & -1.571538000 & -0.253743000 \\
\hline $\mathrm{H}$ & -5.183182000 & -2.515836000 & -0.307245000 \\
\hline C & -7.315239000 & -1.252956000 & -1.751762000 \\
\hline $\mathrm{H}$ & -6.917747000 & -0.739509000 & -2.634694000 \\
\hline $\mathrm{H}$ & -8.404245000 & -1.332618000 & -1.860978000 \\
\hline $\mathrm{H}$ & -6.905712000 & -2.268289000 & -1.744835000 \\
\hline C & 2.371556000 & 4.324316000 & 0.220674000 \\
\hline $\mathrm{H}$ & 2.464841000 & 5.397964000 & 0.275805000 \\
\hline C & -1.663780000 & -3.038022000 & 1.199392000 \\
\hline $\mathrm{H}$ & -0.942097000 & -2.226340000 & 1.273736000 \\
\hline $\mathrm{H}$ & -1.121280000 & -3.990798000 & 1.215605000 \\
\hline $\mathrm{H}$ & -2.318167000 & -3.007574000 & 2.079663000 \\
\hline C & 7.700826000 & -0.930562000 & 0.281218000 \\
\hline $\mathrm{H}$ & 7.641738000 & -0.222400000 & 1.115781000 \\
\hline $\mathrm{H}$ & 8.752014000 & -1.018349000 & -0.021531000 \\
\hline $\mathrm{H}$ & 7.377433000 & -1.908792000 & 0.651272000 \\
\hline $\mathrm{C}$ & -3.444965000 & -4.152371000 & -0.128482000 \\
\hline $\mathrm{H}$ & -4.117433000 & -4.179936000 & 0.737449000 \\
\hline
\end{tabular}




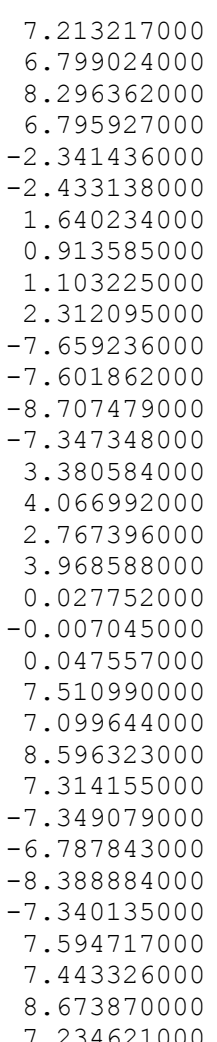

7.234621000

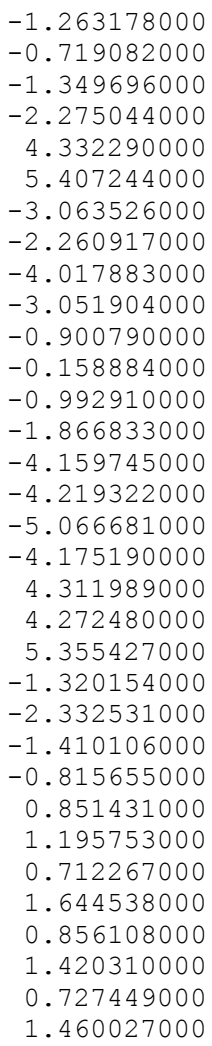

1.854489000

2.710554000

1.995675000

1.863939000

$-0.074418000$

$-0.103129000$

$-1.191494000$

$-1.305044000$

$-1.197949000$

$-2.057848000$

$-0.284202000$

$-1.088471000$

0.021298000

$-0.693228000$

0.204875000

$-0.647631000$

0.196229000

1.129468000

$-0.878577000$

$-1.973755000$

$-0.558892000$

$-0.655544000$

$-0.718658000$

$-0.534193000$

$-1.608032000$

1.477161000

2.353760000

1.789291000

0.719853000

0.578193000

$-0.349827000$

0.708172000

1.419636000

\section{$1_{p_{y}}^{+}$}

\begin{tabular}{|c|c|}
\hline $\mathrm{Ni}$ & -0.011632000 \\
\hline 0 & -1.307605000 \\
\hline 0 & 1.240873000 \\
\hline $\mathrm{N}$ & -2.696000000 \\
\hline $\mathrm{N}$ & 2.662793000 \\
\hline $\mathrm{N}$ & 1.195210000 \\
\hline $\mathrm{N}$ & -1.186203000 \\
\hline $\mathrm{C}$ & 2.496363000 \\
\hline $\mathrm{C}$ & 1.365593000 \\
\hline $\mathrm{C}$ & -2.619095000 \\
\hline $\mathrm{C}$ & -1.359376000 \\
\hline $\mathrm{C}$ & -3.342683000 \\
\hline C & -5.445547000 \\
\hline $\mathrm{C}$ & -3.372232000 \\
\hline $\mathrm{C}$ & 4.731944000 \\
\hline $\mathrm{H}$ & 5.269253000 \\
\hline $\mathrm{C}$ & -2.621606000 \\
\hline $\mathrm{C}$ & -4.745054000 \\
\hline $\mathrm{H}$ & -5.249360000 \\
\hline $\mathrm{C}$ & 3.256465000 \\
\hline $\mathrm{C}$ & 5.418430000 \\
\hline $\mathrm{C}$ & 7.514241000 \\
\hline $\mathrm{H}$ & 7.106040000 \\
\hline $\mathrm{H}$ & 8.603816000 \\
\hline $\mathrm{H}$ & 7.281948000 \\
\hline $\mathrm{C}$ & 1.651149000 \\
\hline $\mathrm{H}$ & 2.304222000 \\
\hline $\mathrm{H}$ & 1.063316000 \\
\hline $\mathrm{H}$ & 0.963427000 \\
\hline $\mathrm{C}$ & 2.555315000 \\
\hline $\mathrm{C}$ & -1.900062000 \\
\hline $\mathrm{H}$ & -2.627856000 \\
\hline $\mathrm{H}$ & -1.358892000 \\
\hline $\mathrm{H}$ & -1.185210000 \\
\hline $\mathrm{C}$ & 4.643538000 \\
\hline $\mathrm{H}$ & 5.171439000 \\
\hline $\mathrm{C}$ & 1.609188000 \\
\hline $\mathrm{H}$ & 0.873131000 \\
\hline
\end{tabular}
0.990620000 $-0.210490000$ $-0.306687000$ 2.154092000 2. 088785000 3.657694000 3. 690024000 $-2.911794000$
2. 313813000 $-0.260625000$ 2. 340963000 $-1.494316000$ $-0.325641000$ 0.898790000 0.832226000 1.770878000 $-2.797899000$ 0.868339000 1.778179000 $-1.581484000$ $-0.365309000$ $-1.304852000$ $-2.320728000$ $-1.384552000$ $-0.854537000$ $-2.860300000$ $-2.767117000$ $-3.778066000$ $-2.015367000$ $-0.339608000$ $-2.626825000$ $-2.432147000$ $-3.545378000$ $-1.803506000$ $-1.542255000$ $-2.479310000$ $-3.139891000$ $-0.197856000$ $-0.811955000$ $-0.675020000$ $-0.353957000$ $-0.679740000$ $-0.495628000$ $-0.292736000$ $-0.228630000$ $-0.345921000$ $-0.635657000$ $-0.207839000$ $-0.757261000$ $-0.150069000$ $-0.347636000$ $-0.433042000$ $-0.402764000$ $-1.131238000$ $-0.072604000$ 0.226131000 $-0.355388000$ $-0.307301000$ $-1.326587000$ $-1.321875000$ $-1.238007000$ $-2.298144000$ 1.066073000 1.942048000 1. 176732000 1.057919000 $-0.521797000$ $-2.489491000$ $-3.285963000$ $-2.743281000$ $-2.464864000$ $-0.278479000$ $-0.166313000$ $-1.473236000$ $-1.593954000$ 


\begin{tabular}{|c|c|c|c|}
\hline $\mathrm{H}$ & 1.080805000 & -4.095932000 & -1.386444000 \\
\hline $\mathrm{H}$ & 2.228232000 & -3.180106000 & -2.377184000 \\
\hline $\mathrm{C}$ & 3.280193000 & 3.280206000 & -1.053417000 \\
\hline $\mathrm{H}$ & 4.293911000 & 3.316991000 & -1.412977000 \\
\hline 0 & 3.443128000 & -4.123341000 & -0.111924000 \\
\hline F & 4.083231000 & -4.231040000 & -0.994910000 \\
\hline $\mathrm{H}$ & 2.843844000 & -5.036106000 & -0.027931000 \\
\hline $\mathrm{H}$ & 4.081604000 & -4.071005000 & 0.777477000 \\
\hline & 6.945602000 & -0.448606000 & -0.170526000 \\
\hline c & -2.392282000 & 4.340710000 & -0.485263000 \\
\hline $\mathrm{H}$ & -2.466191000 & 5.412799000 & -0.584197000 \\
\hline & -6.947120000 & -0.439695000 & 0.149888000 \\
\hline c & -3.340398000 & 3.376012000 & -0.534834000 \\
\hline $\mathrm{H}$ & -4.396106000 & 3.446787000 & -0.731136000 \\
\hline & 3.337293000 & 0.836870000 & -0.561850000 \\
\hline c & -4.711620000 & -1.472084000 & -0.517473000 \\
\hline $\mathrm{H}$ & -5.255607000 & -2.403115000 & -0.601524000 \\
\hline 0 & -7.679912000 & -0.979632000 & -1.100876000 \\
\hline $\mathrm{H}$ & -7.548943000 & -0.304015000 & -1.953556000 \\
\hline $\mathrm{H}$ & -8.753276000 & -1.071946000 & -0.899320000 \\
\hline $\mathrm{H}$ & -7.312514000 & -1.967789000 & -1.396241000 \\
\hline c & 2.361212000 & 4.267022000 & -0.923967000 \\
\hline $\mathrm{H}$ & 2.430497000 & 5.328142000 & -1.108020000 \\
\hline 0 & -1.610643000 & -3.135702000 & -0.015412000 \\
\hline $\mathrm{H}$ & -0.873269000 & -2.343414000 & 0.092160000 \\
\hline F & -1.081507000 & -4.065377000 & -0.250418000 \\
\hline $\mathrm{H}$ & -2.125389000 & -3.273877000 & 0.943750000 \\
\hline $\mathrm{C}$ & 7.304868000 & -1.106095000 & 1.182737000 \\
\hline $\mathrm{H}$ & 6.917482000 & -0.513615000 & 2.019695000 \\
\hline $\mathrm{H}$ & 8.392908000 & -1.180228000 & 1.292149000 \\
\hline $\mathrm{H}$ & 6.893516000 & -2.117270000 & 1.267699000 \\
\hline c & -3.590060000 & -3.988870000 & -1.266493000 \\
\hline $\mathrm{H}$ & -4.101989000 & -4.216882000 & -0.324308000 \\
\hline $\mathrm{H}$ & -3.021131000 & -4.880066000 & -1.551343000 \\
\hline $\mathrm{H}$ & -4.346067000 & -3.820466000 & -2.041948000 \\
\hline $\mathrm{C}$ & 0.048907000 & 4.382071000 & 0.010579000 \\
\hline $\mathrm{H}$ & 0.143668000 & 4.504699000 & 1.095536000 \\
\hline $\mathrm{H}$ & 0.022062000 & 5.367704000 & -0.457467000 \\
\hline $\mathrm{C}$ & -7.159004000 & -1.415258000 & 1.331505000 \\
\hline $\mathrm{H}$ & -6.770814000 & -2.414599000 & 1.109107000 \\
\hline $\mathrm{H}$ & -8.227481000 & -1.515670000 & 1.554240000 \\
\hline $\mathrm{H}$ & -6.654990000 & -1.051492000 & 2.234345000 \\
\hline $\mathrm{C}$ & 7.611788000 & 0.938254000 & -0.224587000 \\
\hline $\mathrm{H}$ & 7.408769000 & 1.450256000 & -1.172828000 \\
\hline $\mathrm{H}$ & 8.697724000 & 0.830616000 & -0.136859000 \\
\hline $\mathrm{H}$ & 7.280054000 & 1.582671000 & 0.598521000 \\
\hline $\mathrm{C}$ & -7.570888000 & 0.916907000 & 0.525148000 \\
\hline $\mathrm{H}$ & -7.118224000 & 1.338723000 & 1.430628000 \\
\hline $\mathrm{H}$ & -8.640134000 & 0.790029000 & 0.723337000 \\
\hline $\mathrm{H}$ & -7.473148000 & 1.647709000 & -0.286642000 \\
\hline $\mathrm{N}$ & 0.055119000 & 0.455662000 & 1.788231000 \\
\hline $\mathrm{C}$ & -1.000349000 & -0.151949000 & 2.356231000 \\
\hline C & 1.222070000 & 0.494150000 & 2.453107000 \\
\hline $\mathrm{C}$ & 1.377513000 & -0.067491000 & 3.715118000 \\
\hline $\mathrm{C}$ & -0.926303000 & -0.740230000 & 3.614503000 \\
\hline $\mathrm{H}$ & 2.340526000 & -0.018395000 & 4.211876000 \\
\hline $\mathrm{H}$ & 2.050202000 & 0.968839000 & 1.938406000 \\
\hline $\mathrm{H}$ & -1.801296000 & -1.226626000 & 4.032064000 \\
\hline $\mathrm{H}$ & -1.914285000 & -0.172958000 & 1.774031000 \\
\hline 1 & 0.283870000 & -0.700162000 & 4.306832000 \\
\hline $\mathrm{H}$ & 0.374570000 & -1.157084000 & 5.287586000 \\
\hline
\end{tabular}

\section{$1_{\mathrm{Py}^{+}}$}

$\mathrm{Ni} \quad 0.002891000$

1.027532000

0.199379000

0.465408000

1.414757000

1.419879000

2. 979846000

2.970339000

$-1.090449000$

1.860026000

$-0.221355000$

-0.160717000
0.887636000
0.929622000
-1.335694000
-1.475208000
-2.002829000
-1.934994000
2.789437000
-1.251810000
0.487594000 


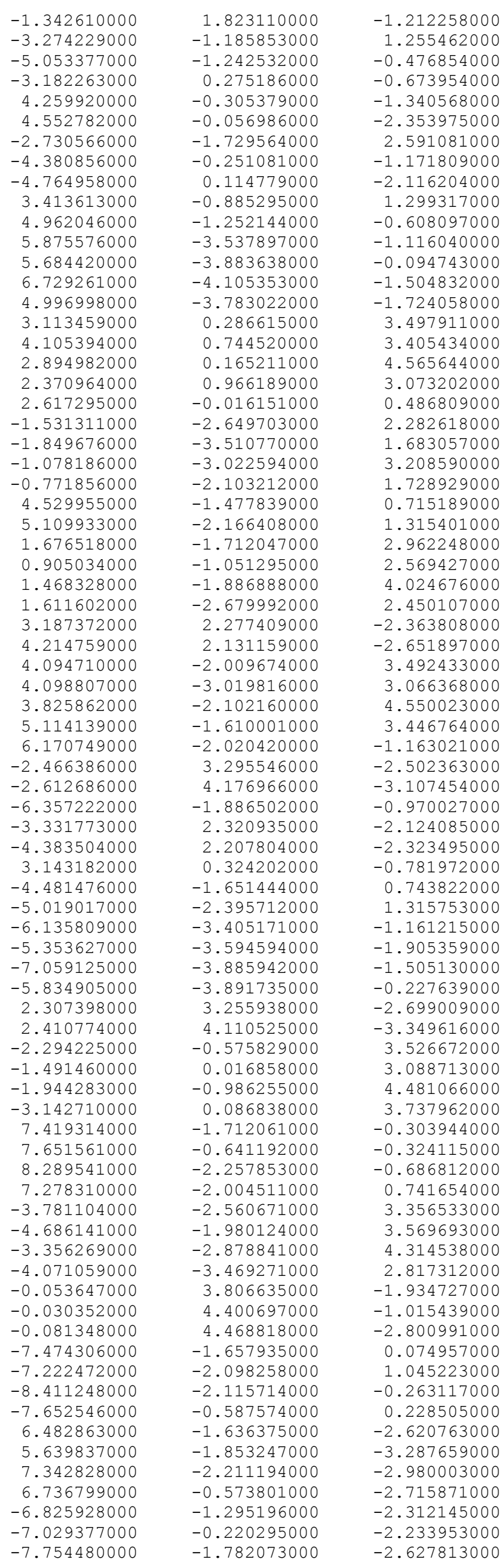




$\begin{array}{lr}\mathrm{H} & -6.086361000 \\ \mathrm{~N} & -0.113441000 \\ \mathrm{~N} & 0.103017000 \\ \mathrm{C} & 0.432355000 \\ \mathrm{C} & 0.426472000 \\ \mathrm{C} & 0.066068000 \\ \mathrm{C} & -0.271438000 \\ \mathrm{C} & -0.238585000 \\ \mathrm{C} & -1.285416000 \\ \mathrm{C} & 1.008188000 \\ \mathrm{C} & 1.001356000 \\ \mathrm{C} & -1.384452000 \\ \mathrm{H} & 1.933933000 \\ \mathrm{H} & 1.930066000 \\ \mathrm{H} & -2.352923000 \\ \mathrm{H} & -2.152126000 \\ \mathrm{H} & -0.557863000 \\ \mathrm{H} & 0.048894000 \\ \mathrm{H} & 0.699425000 \\ \mathrm{H} & 0.707544000 \\ \mathrm{H} & -0.493512000 \\ \mathrm{C} & -0.219127000 \\ \mathrm{H} & -0.259778000 \\ \mathrm{H} & \end{array}$

-1.452143000
2.708568000
-0.784717000
-1.929905000
-3.157686000
-3.200255000
-2.008746000
-0.826326000
3.013714000
3.270325000
4.173747000
3.905627000
4.598886000
2.948882000
4.117067000
2.496667000
-1.992468000
-4.142835000
-4.056580000
-1.838294000
0.126859000
4.497796000
5.192073000

$1^{2+}$

$\mathrm{Ni} \quad-0.028650000$

o $\quad-1.290933000$

1. 329938000

$-2.716553000$

2.560847000

1.117802000

$-1.273703000$

2.855429000

1. 264227000

$-2.541347000$

$-1.382971000$

$-3.209494000$

$-5.309947000$

$-3.329822000$

4.452642000

4.879771000

$-2.422219000$

$-4.658276000$

$-5.225718000$

3. 386314000

5.187815000

6.472485000

6.209945000

7. 450927000

5.737259000

2.352307000

3.159272000

2.009465000

1.518669000

2.570650000

$-1.382023000$

$-1.887454000$

$-0.794993000$

$-0.694533000$

4.635209000

5.239486000

1.699325000

0.878315000

1. 324136000

2. 040435000

3.204560000

4. 242666000

3. 948797000

4. 292676000

3.534593000

4. 813921000

6.550028000

$-2.507444000$
1.052903000

$-0.332952000$

$-0.252430000$

2. 093893000

2.178114000

3. 696057000

3. 645560000

$-2.659848000$

2. 355254000

$-0.377683000$

2. 306149000

$-1.669724000$

$-0.477686000$

0.832091000

0.840831000

1.703652000

$-2.975794000$

0.764747000

1.667707000

$-0.366156000$

$-1.666084000$

$-1.786808000$

$-1.450086000$

$-2.202602000$

$-1.734206000$

$-3.077570000$

$-1.501403000$

$-0.256119000$

$-3.025892000$

$-3.031351000$

$-3.946413000$

$-2.178898000$

$-1.460019000$

$-2.351240000$

$-3.307380000$

$-2.607620000$

$-4.177924000$

$-3.647624000$

3. 415505000

3. 517542000

$-3.724147000$

$-4.169067000$

$-4.536412000$

$-3.322192000$

$-0.508714000$

4. 273083000
$-1.457233000$

$-2.621174000$
$-3.106483000$

1. 294021000

$-1.362958000$

$-0.746527000$

$-1.401166000$

$-2.747144000$

$-3.391174000$

$-2.659530000$

1.871842000

1. 770864000

2. 829959000

2. 936508000

3. 186040000

1. 298924000

3.377536000

1. 475969000

$-4.437539000$

$-3.286190000$

$-0.858803000$

0.294510000

$-3.113796000$

3. 424289000

4. 258414000
0.480899000

0.391154000

0.672903000

0.189257000

$-0.064411000$

0.450077000

0.600113000

1.325761000

0.329102000

0.119138000

0.415739000

$-0.045423000$

$-0.565481000$

$-0.046222000$

$-0.793868000$

$-1.285640000$

0.084666000

$-0.405017000$

$-0.573180000$

0.540066000

$-0.761169000$

$-2.460650000$

$-1.995886000$

$-2.936471000$

$-3.243032000$

2. 719760000

3. 294312000

3. 281106000

2. 648070000

0.359090000

$-1.065832000$

$-2.037788000$

$-0.987816000$

$-1.038250000$

$-0.043726000$

0.042044000

0.529716000

0.394061000

1. 078085000

$-0.454369000$

$-0.170201000$

$-0.436004000$

1. 545788000

0.605597000

2. 150088000

2.084887000

$-1.427317000$

0.515371000 


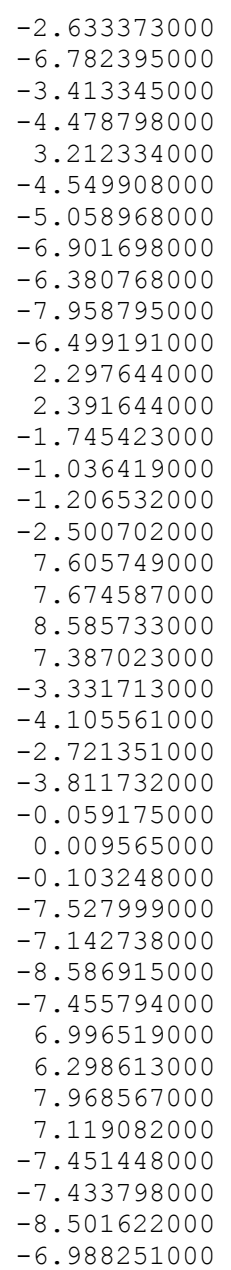

$-6.988251000$
5.336874000 $-0.580893000$ 3. 304849000 3.384925000 0.922937000 $-1.657838000$ $-2.602587000$ $-1.372802000$ $-0.859007000$ $-1.457566000$ $-2.386925000$ 4.364574000 5.438572000 $-3.058761000$ $-2.249009000$ $-4.007728000$ $-3.036133000$ $-0.855998000$ $-0.064300000$ $-0.958795000$ $-1.798707000$ $-4.213155000$ $-4.247770000$ $-5.114390000$ $-4.268475000$ 4. 327864000 4. 309874000 5.363452000 $-1.348438000$ $-2.362852000$ $-1.430516000$ $-0.818359000$ 0.772111000 1. 058720000 0.600640000 1. 616103000 0.793290000 1.386976000 0.650777000 1. 371788000
0.649686000

$-0.942702000$

0.258109000

0.133389000

$-0.203438000$

$-0.362904000$

$-0.483897000$

$-2.273675000$

$-3.088475000$

$-2.545247000$

$-2.192935000$

0.153938000

0.210319000

1. 477377000

1. 648420000

1.559272000

2.270795000

$-0.342105000$

0.411335000

$-0.818908000$

0.168156000

$-0.057817000$

0.717120000

0.051947000

$-1.041002000$

1. 020808000

2.113639000

0.680000000

0.183476000

0.322769000

$-0.081587000$

1.138975000

$-2.155392000$

$-2.950719000$

$-2.625936000$

$-1.466188000$

$-1.127527000$

$-0.205904000$

$-1.396840000$

$-1.935513000$
2

$\begin{array}{lr}\mathrm{Pd} & 0.009243000 \\ \mathrm{O} & 1.469271000 \\ \mathrm{O} & -1.522774000 \\ \mathrm{~N} & 2.745981000 \\ \mathrm{~N} & -2.678739000 \\ \mathrm{~N} & -1.172477000 \\ \mathrm{~N} & 1.250528000 \\ \mathrm{C} & -3.035406000 \\ \mathrm{C} & -1.365843000 \\ \mathrm{C} & 2.711066000 \\ \mathrm{C} & 1.420797000 \\ \mathrm{C} & 3.465136000 \\ \mathrm{C} & 5.439186000 \\ \mathrm{C} & 3.394086000 \\ \mathrm{C} & -4.593577000 \\ \mathrm{H} & -4.957561000 \\ \mathrm{C} & 2.778774000 \\ \mathrm{C} & 4.713046000 \\ \mathrm{H} & 5.163052000 \\ \mathrm{C} & -3.555695000 \\ \mathrm{C} & -5.348657000 \\ \mathrm{C} & -6.671009000 \\ \mathrm{H} & -6.417233000 \\ \mathrm{H} & -7.645079000 \\ \mathrm{H} & -5.918498000 \\ \mathrm{C} & -2.748389000 \\ \mathrm{H} & -3.665525000 \\ \mathrm{H} & -2.373243000 \\ & \end{array}$

0.890752000 $-0.543849000$ $-0.455051000$ 2. 026663000 2.082136000 3. 605054000 3. 575861000 $-2.874209000$ 2. 257390000 $-0.461531000$ 2. 223868000 $-1.679579000$ $-0.397010000$ 0.755806000 0.822725000 1. 744546000 $-3.042816000$ 0.771398000 1. 715227000 $-1.566917000$ $-0.334779000$ $-1.409120000$ $-2.413785000$ $-1.467182000$ $-1.116905000$ $-2.643364000$ $-2.352903000$ $-3.568159000$
$-0.574389000$ $-0.665928000$ $-0.876146000$ $-0.172506000$ $-0.097234000$ $-0.468342000$ $-0.491335000$ $-0.988735000$ $-0.380665000$ $-0.261271000$ $-0.381706000$ $-0.084328000$ 0.670172000 0.030132000 0.717694000 1. 153662000 $-0.303237000$ 0.511047000 0.787522000 $-0.358407000$ 0.821846000 2. 677418000 2. 323867000 3. 179786000 3. 418709000 $-2.492952000$ $-3.020563000$ $-2.949472000$ 


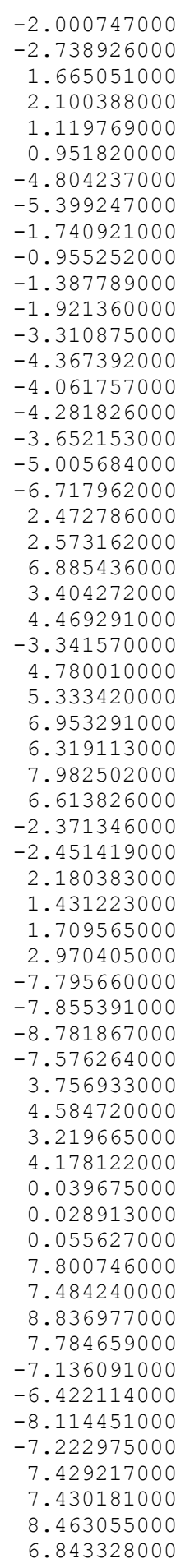

6.843328000

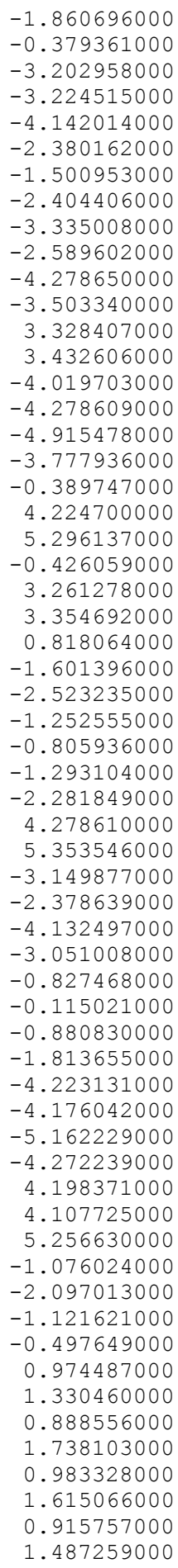

$-2.632389000$

$-0.398425000$

0.760276000

1. 767113000

0.604052000

0.707339000

0.244360000

0.289837000

$-0.280270000$

$-0.387018000$

$-0.716313000$

0.788707000

$-0.045656000$

0.128623000

$-0.881798000$

0.160941000

$-1.362340000$

$-1.385177000$

1. 515229000

$-0.391237000$

$-0.471316000$

1.187417000

$-0.192154000$

$-0.078071000$

0.084954000

0.349593000

0.475589000

2. 493138000

3. 267523000

2.872116000

2. 337764000

$-0.276054000$

$-0.325040000$

$-1.727127000$

$-1.902673000$

$-1.858097000$

$-2.482329000$

0.495241000

$-0.335747000$

0.973946000

0.072893000

$-0.132690000$

$-0.850795000$

$-0.307055000$

0.878475000

$-1.006828000$

$-2.100060000$

$-0.737322000$

0.123284000

$-0.113559000$

0.481908000

$-0.807737000$

2. 094850000

2.847073000

2.581263000

1. 312328000

1. 486545000

0.589977000

1.843976000

2.264565000
0.911992000 $-0.520903000$ $-0.492236000$ 2. 057508000 2. 079527000 3. 612344000 3. 599524000 $-2.962398000$
$-0.372693000$

$-0.272703000$

$-0.501561000$

0.004127000

$-0.063580000$

$-0.318649000$

$-0.255342000$

$-0.683249000$ 


\begin{tabular}{|c|c|c|}
\hline-1.392765000 & 2.267151000 & -0.282954000 \\
\hline 2.713659000 & -0.442445000 & -0.061714000 \\
\hline 1.417754000 & 2.251648000 & -0.231761000 \\
\hline 3.465040000 & -1.679089000 & 0.039093000 \\
\hline 5.540012000 & -0.383190000 & 0.358775000 \\
\hline 3.428755000 & 0.798045000 & 0.084781000 \\
\hline-4.746128000 & 0.824822000 & 0.4350070 \\
\hline-5.219888000 & 1.749595000 & 0.728995000 \\
\hline 2.733410000 & -3.032408000 & -0.01508300 \\
\hline 4.809497000 & 0.801038000 & 0.288840000 \\
\hline 5.338015000 & 1.736197000 & 0.390495000 \\
\hline-3.517082000 & -1.628368000 & -0.27970600 \\
\hline-5.493021000 & -0.351629000 & 0.470416000 \\
\hline-7.110649000 & -1.321617000 & 2.12525200 \\
\hline-6.789371000 & -2.343724000 & 1.90095800 \\
\hline-8.159576000 & -1.364055000 & 2.439464000 \\
\hline-6.515108000 & -0.957873000 & 2.970008000 \\
\hline-2.263194000 & -2.850963000 & -2.10742200 \\
\hline-3.039965000 & -2.598554000 & -2.838840000 \\
\hline-1.829555000 & -3.814865000 & -2.39709900 \\
\hline-1.477723000 & -2.095420000 & -2.156541000 \\
\hline-2.730082000 & -0.410095000 & -0.257617000 \\
\hline 1.791849000 & -3.131516000 & 1.211883000 \\
\hline 2.369306000 & -3.107930000 & 2.14343400 \\
\hline 1.238144000 & -4.076706000 & 1.180074000 \\
\hline 1.069484000 & -2.314416000 & 1.230219000 \\
\hline-4.848145000 & -1.549124000 & 0.08274000 \\
\hline-5.436335000 & -2.456000000 & 0.084632000 \\
\hline-1.754588000 & -3.332628000 & 0.330546000 \\
\hline-0.965345000 & -2.582815000 & 0.34605800 \\
\hline-1.308378000 & -4.294428000 & 0.051908000 \\
\hline-2.167163000 & -3.432637000 & 1.341377000 \\
\hline-3.355537000 & 3.329750000 & 0.00761100 \\
\hline-4.414899000 & 3.446366000 & 0.148061000 \\
\hline-3.887768000 & -4.116224000 & -0.70081000 \\
\hline-4.320854000 & -4.300882000 & 0.28901600 \\
\hline-3.381763000 & -5.036458000 & -1.010108000 \\
\hline-4.702835000 & -3.936312000 & -1.411514000 \\
\hline-6.963946000 & -0.385880000 & 0.89997400 \\
\hline 2.429440000 & 4.260205000 & -0.045403000 \\
\hline 2.509728000 & 5.336382000 & -0.031509000 \\
\hline 7.057351000 & -0.409419000 & 0.57442200 \\
\hline 3.373112000 & 3.306586000 & 0.124279000 \\
\hline 4.420607000 & 3.424263000 & 0.332679000 \\
\hline-3.406166000 & 0.824077000 & 0.04828300 \\
\hline 4.830794000 & -1.599207000 & 0.231450000 \\
\hline 5.397674000 & -2.517160000 & 0.298034000 \\
\hline 7.370243000 & -1.183537000 & 1.878952000 \\
\hline 6.906698000 & -0.697212000 & 2.744509000 \\
\hline 8.453175000 & -1.214878000 & 2.043324000 \\
\hline 7.010950000 & -2.216743000 & 1.839661000 \\
\hline-2.405520000 & 4.279498000 & -0.152354000 \\
\hline-2.484861000 & 5.355644000 & -0.170442000 \\
\hline 1.926597000 & -3.185769000 & -1.32955100 \\
\hline 1.133006000 & -2.443684000 & -1.412067000 \\
\hline 1.469758000 & -4.181636000 & -1.36021700 \\
\hline 2.585832000 & -3.093117000 & -2.20076600 \\
\hline-7.821878000 & -0.928287000 & -0.269871000 \\
\hline-7.737904000 & -0.281842000 & -1.15058400 \\
\hline-8.875923000 & -0.965996000 & 0.02738600 \\
\hline-7.524428000 & -1.939765000 & -0.563624000 \\
\hline 3.718820000 & -4.217159000 & 0.05691300 \\
\hline 4.418368000 & -4.221931000 & -0.78711000 \\
\hline 3.153673000 & -5.154035000 & 0.019894000 \\
\hline 4.294817000 & -4.223428000 & 0.98927200 \\
\hline 0.029215000 & 4.244465000 & -0.77518600 \\
\hline 0.058247000 & 4.220021000 & -1.870675000 \\
\hline 0.026101000 & 5.283112000 & -0.44042800 \\
\hline 7.726514000 & -1.126399000 & -0.62411200 \\
\hline 7.375529000 & -2.157313000 & -0.733707000 \\
\hline 8.812393000 & -1.158848000 & -0.48037800 \\
\hline 7.521185000 & -0.597900000 & -1.56172800 \\
\hline-7.499160000 & 1.005299000 & 1.286229000 \\
\hline-6.951048000 & 1.433526000 & 2.13391400 \\
\hline-8.549186000 & 0.924683000 & 1.58452300 \\
\hline-7.452988000 & 1.708418000 & 0.44579000 \\
\hline 7.662808000 & 1.001625000 & 0.69293400 \\
\hline
\end{tabular}




$\begin{array}{lrrr}\mathrm{H} & 7.501764000 & 1.593461000 & -0.216148000 \\ \mathrm{H} & 8.744292000 & 0.925452000 & 0.843706000 \\ \mathrm{H} & 7.252985000 & 1.551473000 & 1.548682000\end{array}$

\section{$2^{2+}$}

\begin{tabular}{|c|c|c|c|}
\hline $\mathrm{Pd}$ & 0.012722000 & 0.903832000 & -0.569937000 \\
\hline 0 & 1.500293000 & -0.539745000 & -0.600671000 \\
\hline 0 & -1.539193000 & -0.473204000 & -0.769698000 \\
\hline $\mathrm{N}$ & 2.750513000 & 2.042573000 & -0.190171000 \\
\hline $\mathrm{N}$ & -2.670769000 & 2.103577000 & -0.099280000 \\
\hline $\mathrm{N}$ & -1.170618000 & 3.615154000 & -0.518161000 \\
\hline $\mathrm{N}$ & 1.262830000 & 3.582321000 & -0.559887000 \\
\hline C & -3.086007000 & -2.876488000 & -1.061519000 \\
\hline $\mathrm{C}$ & -1.357986000 & 2.281599000 & -0.406144000 \\
\hline C & 2.719535000 & -0.474921000 & -0.238786000 \\
\hline $\mathrm{C}$ & 1.423967000 & 2.246179000 & -0.421153000 \\
\hline C & 3.472483000 & -1.724606000 & -0.079102000 \\
\hline C & 5.416336000 & -0.417951000 & 0.696476000 \\
\hline $\mathrm{C}$ & 3.399323000 & 0.786860000 & 0.047178000 \\
\hline $\mathrm{C}$ & -4.554499000 & 0.842133000 & 0.754651000 \\
\hline $\mathrm{H}$ & -4.944163000 & 1.750408000 & 1.192657000 \\
\hline $\mathrm{C}$ & 2.788232000 & -3.074442000 & -0.328916000 \\
\hline C & 4.688419000 & 0.783825000 & 0.532866000 \\
\hline $\mathrm{H}$ & 5.167639000 & 1.711750000 & 0.807943000 \\
\hline $\mathrm{C}$ & -3.582632000 & -1.595501000 & -0.380213000 \\
\hline $\mathrm{C}$ & -5.315067000 & -0.346999000 & 0.862651000 \\
\hline C & -6.586073000 & -1.452716000 & 2.710282000 \\
\hline $\mathrm{H}$ & -6.370653000 & -2.454266000 & 2.326081000 \\
\hline $\mathrm{H}$ & -7.552190000 & -1.496227000 & 3.223251000 \\
\hline $\mathrm{H}$ & -5.819365000 & -1.194413000 & 3.448431000 \\
\hline $\mathrm{C}$ & -2.774551000 & -2.575483000 & -2.551981000 \\
\hline $\mathrm{H}$ & -3.666147000 & -2.211038000 & -3.074301000 \\
\hline $\mathrm{H}$ & -2.454568000 & -3.499531000 & -3.044349000 \\
\hline $\mathrm{H}$ & -1.975297000 & -1.839592000 & -2.663269000 \\
\hline $\mathrm{C}$ & -2.746845000 & -0.390326000 & -0.374738000 \\
\hline $\mathrm{C}$ & 1.678455000 & -3.263630000 & 0.740422000 \\
\hline $\mathrm{H}$ & 2.108744000 & -3.288934000 & 1.747758000 \\
\hline $\mathrm{H}$ & 1.169804000 & -4.217964000 & 0.567934000 \\
\hline $\mathrm{H}$ & 0.932379000 & -2.468376000 & 0.699222000 \\
\hline C & -4.806976000 & -1.520937000 & 0.249279000 \\
\hline $\mathrm{H}$ & -5.422184000 & -2.408116000 & 0.290357000 \\
\hline $\mathrm{C}$ & -1.812572000 & -3.406909000 & -0.353570000 \\
\hline $\mathrm{H}$ & -0.978711000 & -2.713113000 & -0.451026000 \\
\hline $\mathrm{H}$ & -1.521254000 & -4.358166000 & -0.811688000 \\
\hline $\mathrm{H}$ & -1.999268000 & -3.590464000 & 0.710620000 \\
\hline $\mathrm{C}$ & -3.305976000 & 3.353132000 & -0.052418000 \\
\hline $\mathrm{H}$ & -4.360448000 & 3.467182000 & 0.132298000 \\
\hline $\mathrm{C}$ & -4.153421000 & -3.988281000 & -1.015292000 \\
\hline $\mathrm{H}$ & -4.381827000 & -4.301048000 & 0.009882000 \\
\hline $\mathrm{H}$ & -3.773015000 & -4.867451000 & -1.543367000 \\
\hline $\mathrm{H}$ & -5.084179000 & -3.689658000 & -1.510451000 \\
\hline C & -6.658344000 & -0.389451000 & 1.580839000 \\
\hline $\mathrm{C}$ & 2.487140000 & 4.233715000 & -0.448000000 \\
\hline $\mathrm{H}$ & 2.591677000 & 5.303370000 & -0.550353000 \\
\hline C & 6.846714000 & -0.433930000 & 1.223293000 \\
\hline $\mathrm{C}$ & 3.415277000 & 3.277940000 & -0.219241000 \\
\hline $\mathrm{H}$ & 4.479575000 & 3.378147000 & -0.097357000 \\
\hline C & -3.335726000 & 0.854259000 & 0.114638000 \\
\hline $\mathrm{C}$ & 4.775772000 & -1.636451000 & 0.359998000 \\
\hline $\mathrm{H}$ & 5.343329000 & -2.547400000 & 0.482074000 \\
\hline $\mathrm{C}$ & 6.886078000 & -1.280909000 & 2.524934000 \\
\hline $\mathrm{H}$ & 6.245797000 & -0.846074000 & 3.299826000 \\
\hline $\mathrm{H}$ & 7.912434000 & -1.305558000 & 2.904969000 \\
\hline $\mathrm{H}$ & 6.570181000 & -2.315008000 & 2.357601000 \\
\hline $\mathrm{C}$ & -2.368712000 & 4.293582000 & -0.312297000 \\
\hline $\mathrm{H}$ & -2.451584000 & 5.367689000 & -0.382367000 \\
\hline $\mathrm{C}$ & 2.184444000 & -3.141121000 & -1.756712000 \\
\hline $\mathrm{H}$ & 1.384748000 & -2.415104000 & -1.904871000 \\
\hline $\mathrm{H}$ & 1.770568000 & -4.141571000 & -1.919699000 \\
\hline $\mathrm{H}$ & 2.957114000 & -2.974217000 & -2.515488000 \\
\hline
\end{tabular}




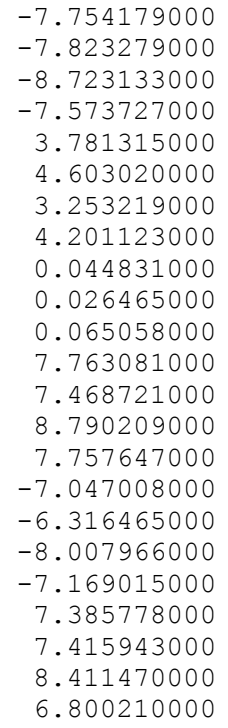

$-0.796816000$

$-0.071211000$

$-0.830053000$

$-1.785603000$

$-4.246730000$

$-4.175997000$

$-5.184066000$

$-4.322315000$

4.236581000

4.203478000

5. 276576000

$-1.087779000$

$-2.116188000$

$-1.114528000$

$-0.512225000$

0.963663000

1.297915000

0.862659000

1. 747657000

0.973877000

1. 611258000

0.893886000

1.475626000
0.557336000

$-0.260122000$

1. 065910000

0.124971000

$-0.190334000$

$-0.911902000$

$-0.387725000$

0.818847000

$-1.035650000$

$-2.129899000$

$-0.706917000$

0.153289000

$-0.076238000$

0.531131000

$-0.778442000$

2. 204782000

2. 950917000

2. 716923000

1. 447777000

1.537276000

0.645398000

1.908058000

2. 316813000

\section{3}

P
O
O
N
N
N
N
C
C
C
C
C
C
C
C
H
C
C
H
C
C
C
H
H
H
C
H
H
H
C
C
H
H
H
H
C
H
C
H
H
H
C

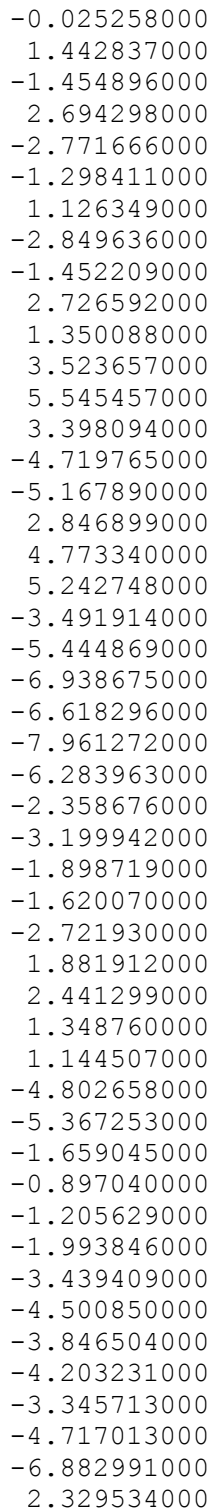

0.855468000

$-0.572192000$

$-0.608809000$

2. 046128000

1. 914044000

3.505312000

3. 560669000

$-3.064908000$

2. 144969000

$-0.445006000$

2.207763000

$-1.644113000$

$-0.322613000$

0.788231000

0.574301000

1. 475898000

$-3.020081000$

0.826865000

1.774952000

$-1.771288000$

$-0.606307000$

$-1.732459000$

$-2.727034000$

$-1.821273000$

$-1.425152000$

$-2.842544000$

$-2.585634000$

$-3.762259000$

$-2.041442000$

$-0.555552000$

$-3.241082000$

$-3.273506000$

$-4.193024000$

$-2.440265000$

$-1.752212000$

$-2.675365000$

$-3.479673000$

$-2.702924000$

$-4.403588000$

$-3.665658000$

3. 139732000

3. 210921000

$-4.241413000$

$-4.502997000$

$-5.125970000$

$-4.032042000$

$-0.705519000$

4.244196000
$-0.327529000$

$-0.447057000$

$-0.549664000$

$-0.017136000$

0.124252000

$-0.107740000$

$-0.149902000$

$-0.795799000$

$-0.111125000$

$-0.200661000$

$-0.156746000$

$-0.158693000$

0.349537000

0.039705000

0.698254000

1. 093133000

$-0.327662000$

0.324295000

0.544044000

$-0.253677000$

0.711876000

2.398157000

2. 071345000

2. 786403000

3. 221642000

$-2.247645000$

$-2.903435000$

$-2.630844000$

$-2.293886000$

$-0.206774000$

0.862574000

1.805723000

0.748825000

0.922867000

0.200973000

0.178537000

0.099360000

0.114520000

$-0.282166000$

1. 127403000

0.232263000

0.387787000

$-0.821975000$

0.181462000

$-1.231732000$

$-1.455583000$

1. 242703000

$-0.045724000$ 


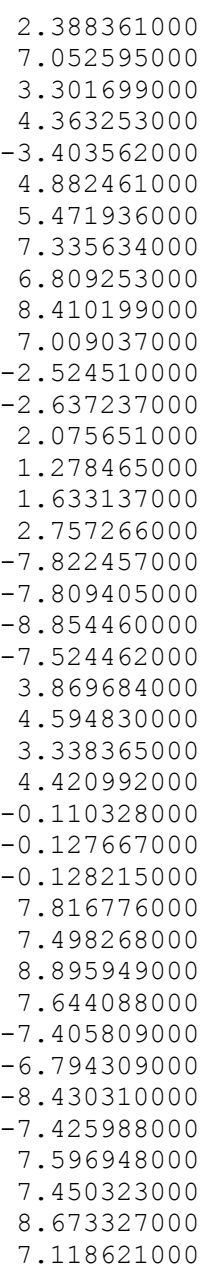

7. 118621000
5.321612000 $-0.318076000$ 3.307085000 3. 441472000 0.622315000 $-1.539839000$ $-2.447092000$ $-1.171516000$ $-0.764302000$ $-1.185105000$ $-2.208166000$ 4.127292000 5.200247000 $-3.111113000$ $-2.370772000$ $-4.109939000$ $-2.954272000$ $-1.166621000$ $-0.448460000$ $-1.254801000$ $-2.141296000$ $-4.175283000$ $-4.091158000$ $-5.125530000$ $-4.233112000$ 4.162222000 4.119839000 5.207127000 $-0.915725000$ $-1.941633000$ $-0.934720000$ $-0.319622000$ 0.640967000 1.011079000 0.522125000 1.408397000 1.099983000 1.751665000 1.054976000 1. 568340000
$-0.038452000$
0.647216000
0.042579000
0.140894000
0.211988000
0.097333000
0.123979000
1. 905979000
2.776883000
2.128649000
1. 774165000
0.086192000
0.102007000
$-1.667428000$
$-1.720608000$
$-1.771596000$
$-2.512945000$
0.103490000
$-0.724592000$
0.466205000
$-0.296461000$
$-0.315553000$
$-1.134320000$
$-0.440807000$
0.630422000
$-0.624947000$
$-1.721804000$
$-0.307628000$
$-0.557547000$
$-0.769540000$
$-0.359023000$
$-1.461037000$
1.777461000
2. 608974000
2.148139000
0.994134000
0.901468000
0.031673000
1.102641000
1. 770054000 


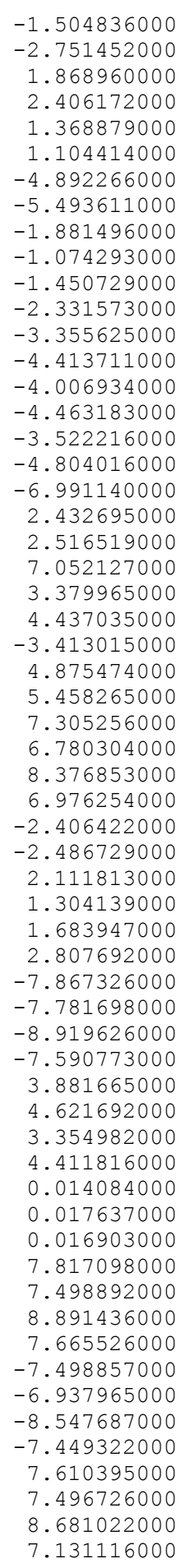

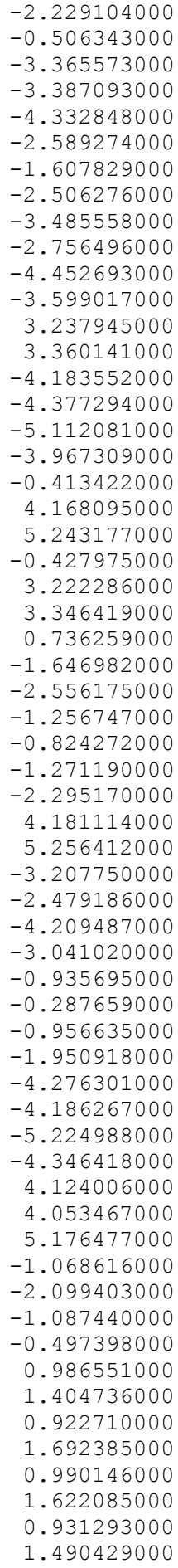

$-2.060981000$

$-0.179000000$

0.977808000

1.933294000

0.852523000

1.028787000

0.151783000

0.145963000

0.422708000

0.487702000

0.138057000

1. 416044000

0.028807000

0.171372000

$-0.674533000$

0.302904000

$-0.993336000$

$-1.395318000$

0.983904000

$-0.170223000$

$-0.215333000$

0.792056000

0.013518000

0.158812000

0.116556000

0.251305000

0.301821000

2.076445000

2. 935628000

2.305417000

1. 968580000

$-0.162206000$

$-0.208041000$

$-1.554710000$

$-1.627343000$

$-1.676335000$

$-2.385666000$

$-0.182431000$

$-1.061936000$

0.122748000

$-0.483780000$

$-0.174205000$

$-0.977982000$

$-0.320098000$

0.782336000

$-0.831900000$

$-1.926724000$

$-0.543136000$

$-0.393198000$

$-0.579376000$

$-0.177849000$

$-1.315989000$

1. 377951000

2.222279000

1. 684830000

0.539978000

1.017126000

0.128116000

1.237713000

1.867160000
$3^{2+}$

$\begin{array}{lr}\text { Pt } & -0.005821000 \\ \mathrm{O} & -1.515567000 \\ \mathrm{O} & 1.533301000 \\ \mathrm{~N} & -2.735905000 \\ \mathrm{~N} & 2.697774000 \\ \mathrm{~N} & 1.194826000 \\ \mathrm{~N} & -1.236404000 \\ \mathrm{C} & 3.117269000\end{array}$

$-0.812896000$

0.644978000

0.617378000

$-1.967326000$

$-1.997192000$

$-3.501030000$

$-3.485011000$

3. 022138000
$-0.478547000$

$-0.512324000$

$-0.585882000$

$-0.132862000$

$-0.076447000$

$-0.529994000$

$-0.555994000$

$-0.872728000$ 


\begin{tabular}{|c|c|c|}
\hline 1.379632000 & -2.167563000 & -0.374404000 \\
\hline-2.751313000 & 0.548442000 & -0.194437000 \\
\hline-1.409740000 & -2.150662000 & -0.388239000 \\
\hline-3.560345000 & 1.771141000 & -0.112467000 \\
\hline-5.431760000 & 0.435672000 & 0.794709000 \\
\hline-3.396032000 & -0.723749000 & 0.119263000 \\
\hline 4.606682000 & -0.772326000 & 0.759673000 \\
\hline 5.004769000 & -1.697819000 & 1.151658000 \\
\hline-2.969836000 & 3.133698000 & -0.505861000 \\
\hline-4.671480000 & -0.746688000 & 0.641643000 \\
\hline-5.111730000 & -1.683357000 & 0.951752000 \\
\hline 3.614852000 & 1.704191000 & -0.260168000 \\
\hline 5.382121000 & 0.404614000 & 0.881916000 \\
\hline 6.726928000 & 1.443081000 & 2.720944000 \\
\hline 6.513121000 & 2.458327000 & 2.373497000 \\
\hline 7.709628000 & 1.459497000 & 3.203127000 \\
\hline 5.982391000 & 1.174999000 & 3.478139000 \\
\hline 2.667346000 & 2.786054000 & -2.339419000 \\
\hline 3.492902000 & 2.399310000 & -2.947608000 \\
\hline 2.356327000 & 3.740829000 & -2.775567000 \\
\hline 1.826173000 & 2.093613000 & -2.409209000 \\
\hline 2.764097000 & 0.507990000 & -0.254674000 \\
\hline-1.868382000 & 3.517027000 & 0.517637000 \\
\hline-2.288085000 & 3.613948000 & 1.525094000 \\
\hline-1.439702000 & 4.485857000 & 0.240073000 \\
\hline-1.062734000 & 2.781784000 & 0.546007000 \\
\hline 4.863187000 & 1.598303000 & 0.318514000 \\
\hline 5.488267000 & 2.479003000 & 0.356675000 \\
\hline 1.940210000 & 3.581975000 & -0.032796000 \\
\hline 1.083411000 & 2.907981000 & -0.036269000 \\
\hline 1.623946000 & 4.541649000 & -0.455686000 \\
\hline 2.245238000 & 3.759045000 & 1.004700000 \\
\hline 3.334629000 & -3.247270000 & -0.077841000 \\
\hline 4.390601000 & -3.367010000 & 0.091688000 \\
\hline 4.229734000 & 4.091458000 & -0.895352000 \\
\hline 4.550499000 & 4.385326000 & 0.110228000 \\
\hline 3.842551000 & 4.991201000 & -1.382497000 \\
\hline 5.107446000 & 3.765831000 & -1.464932000 \\
\hline 6.749133000 & 0.411218000 & 1.559531000 \\
\hline-2.452904000 & -4.150002000 & -0.437527000 \\
\hline-2.549029000 & -5.218278000 & -0.560467000 \\
\hline-6.841157000 & 0.425560000 & 1.38034100 \\
\hline-3.387046000 & -3.209738000 & -0.17 \\
\hline-4.448749000 & -3.322060000 & -0.043091000 \\
\hline 3.365125000 & -0.755919000 & 0.162120000 \\
\hline-4.846984000 & 1.655858000 & 0.372334000 \\
\hline-5.447051000 & 2.550787000 & 0.454529000 \\
\hline-6.859577000 & 1.330267000 & 2.644098000 \\
\hline-6.176958000 & 0.954136000 & 3.413626000 \\
\hline-7.870933000 & 1.338774000 & 3.063102000 \\
\hline-6.586241000 & 2.365522000 & 2.418904000 \\
\hline 2.397716000 & -4.180031000 & -0.359098000 \\
\hline 2.484092000 & -5.250955000 & -0.464902000 \\
\hline-2.390457000 & 3.087442000 & -1.944730000 \\
\hline-1.553772000 & 2.393693000 & -2.03726500 \\
\hline-2.035225000 & 4.086694000 & -2.216641000 \\
\hline-3.162320000 & 2.804643000 & -2.669566000 \\
\hline 7.821222000 & 0.834093000 & 0.516303000 \\
\hline 7.864937000 & 0.128744000 & -0.320512000 \\
\hline 8.803558000 & 0.847076000 & 0.999593000 \\
\hline 7.639119000 & 1.834520000 & 0.112140000 \\
\hline-4.039288000 & 4.246585000 & -0.478936000 \\
\hline-4.868558000 & 4.042420000 & -1.16561200 \\
\hline-3.576893000 & 5.185313000 & -0.79799600 \\
\hline-4.445176000 & 4.416981000 & 0.52428800 \\
\hline-0.018552000 & -4.106677000 & -1.06984900 \\
\hline-0.006672000 & -4.026096000 & -2.16240900 \\
\hline-0.028845000 & -5.160693000 & -0.78877600 \\
\hline-7.827857000 & 0.996635000 & 0.32430300 \\
\hline-7.586513000 & 2.024977000 & 0.03948300 \\
\hline-8.838388000 & 0.999850000 & 0.74547300 \\
\hline-7.841198000 & 0.382995000 & -0.58279800 \\
\hline 7.138795000 & -0.963912000 & 2.134698000 \\
\hline 6.426918000 & -1.312646000 & 2.89216500 \\
\hline 8.114838000 & -0.887269000 & 2.62218500 \\
\hline 7.231975000 & -1.729188000 & 1.35477100 \\
\hline-7.313464 & -0.985229000 & 1 \\
\hline
\end{tabular}


$\mathrm{H} \quad-7.365886000$

$-8.322332000$

$-1.663083000$

$-0.923510000$

0.919348000

$-6.674575000$

$-1.434489000$

2.197614000

2.549389000 


\section{TD-DFT computed electronic excitations}

\section{1}

\begin{tabular}{|c|c|c|c|}
\hline Excited & State & $1:$ & Singlet-A \\
\hline 155 & -> 167 & & 0.10060 \\
\hline 157 & -> 166 & & -0.11250 \\
\hline 157 & -> 167 & & 0.11331 \\
\hline 159 & -> 166 & & -0.11431 \\
\hline 159 & -> 167 & & 0.11046 \\
\hline 160 & -> 166 & & -0.10637 \\
\hline 160 & -> 167 & & 0.10716 \\
\hline 164 & -> 165 & & -0.28436 \\
\hline 164 & -> 166 & & 0.37277 \\
\hline 164 & -> 167 & & -0.32182 \\
\hline 164 & -> 168 & & -0.10495 \\
\hline
\end{tabular}

$2.5370 \mathrm{eV} \quad 488.70 \mathrm{~nm} \quad \mathrm{f}=0.0029 \quad<* * 2>=0.000$

This state for optimization and/or second-order correction.

Total Energy, $\mathrm{E}(\mathrm{TD}-\mathrm{HF} / \mathrm{TD}-\mathrm{KS})=-3239.93384185$

Copying the excited state density for this state as the 1-particle RhocI density.

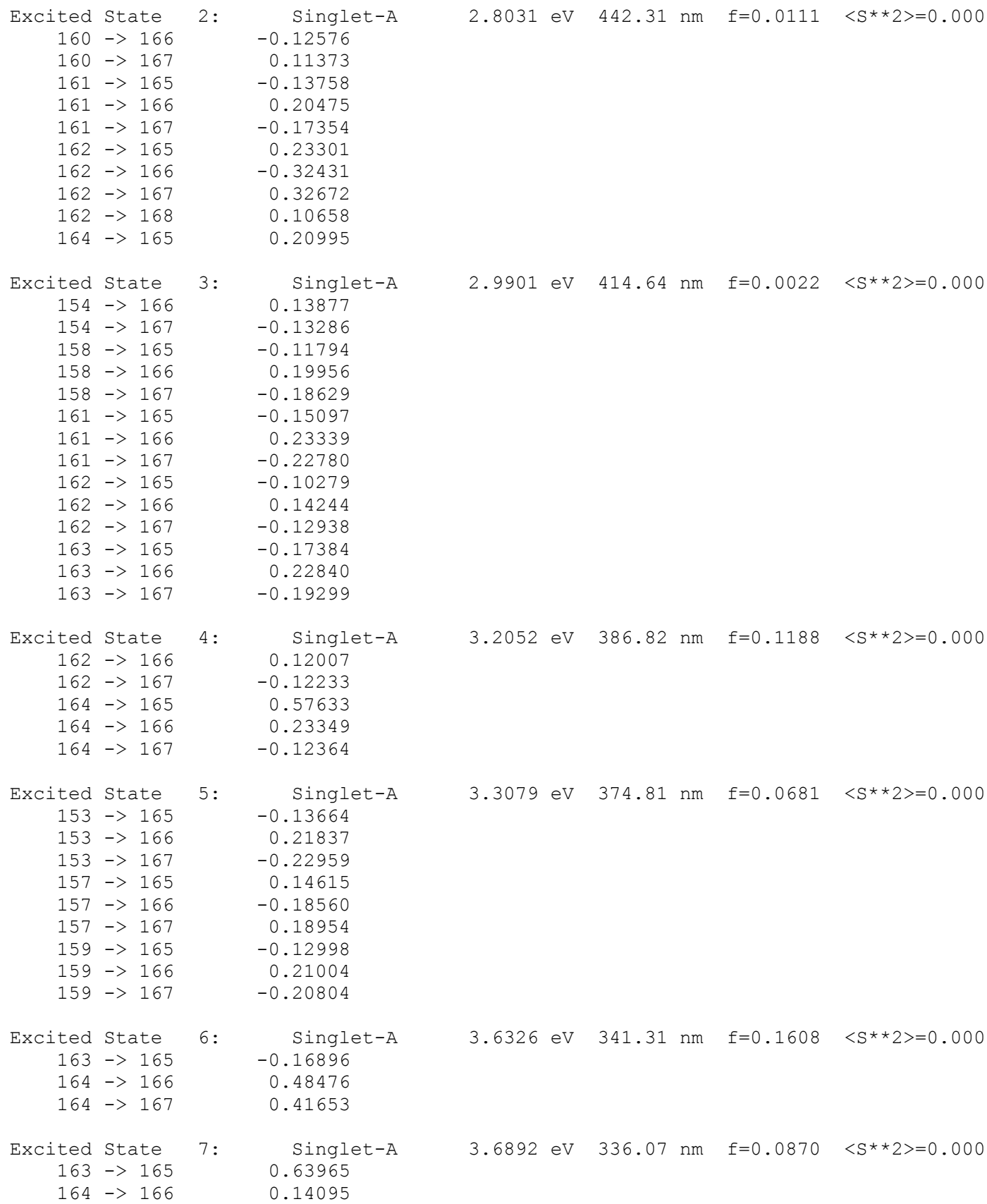$$
0.14095
$$ 


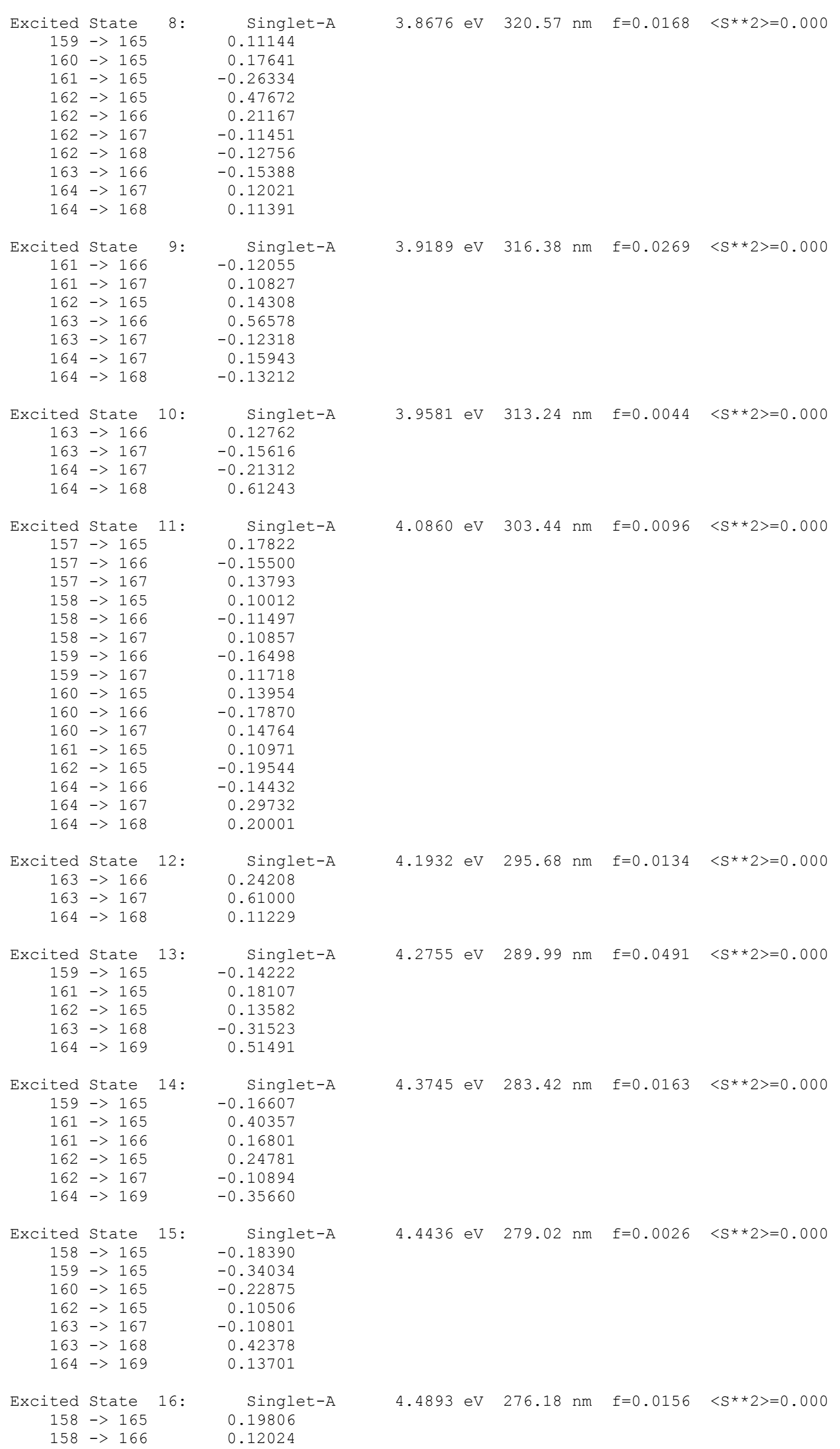




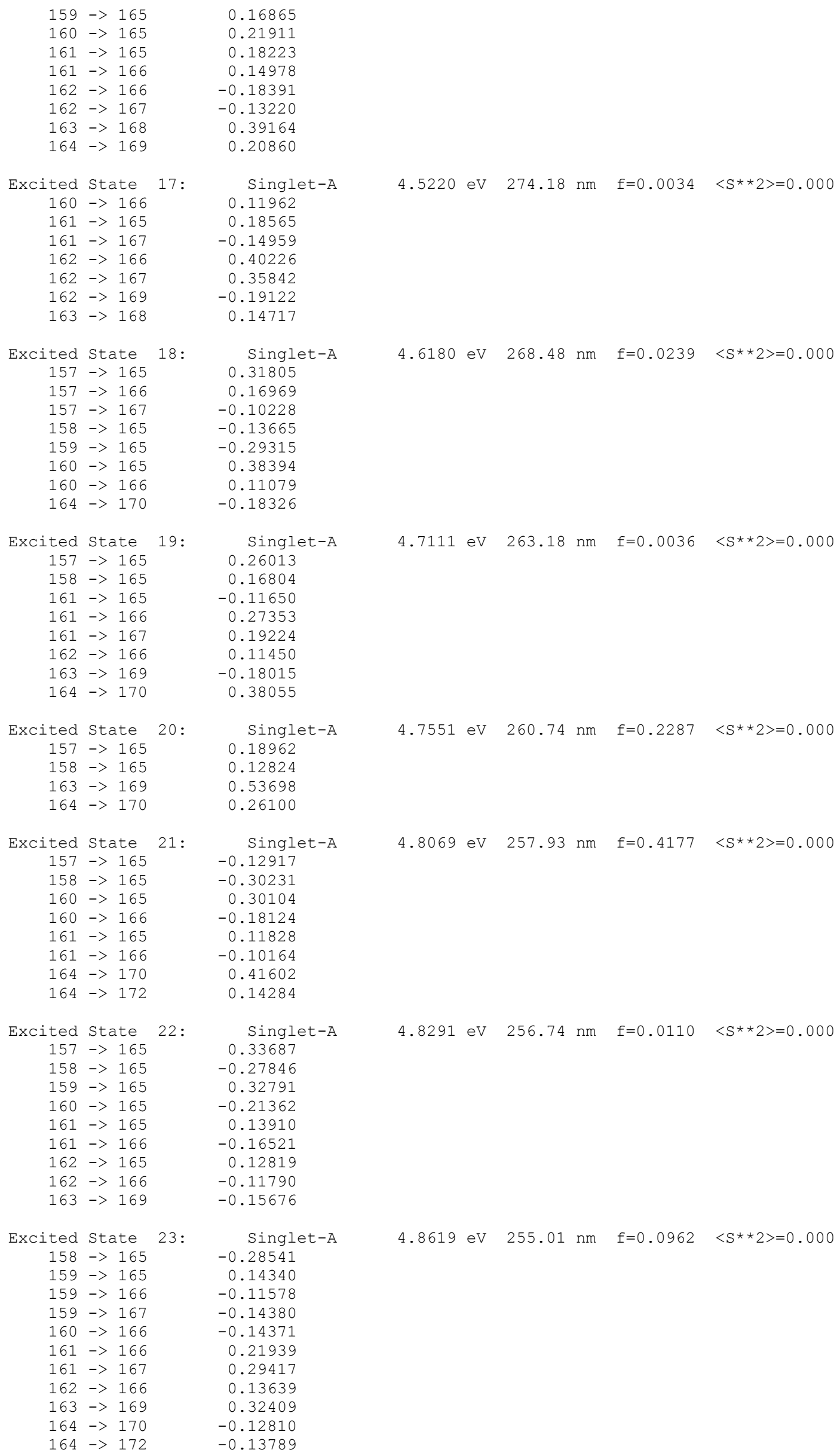




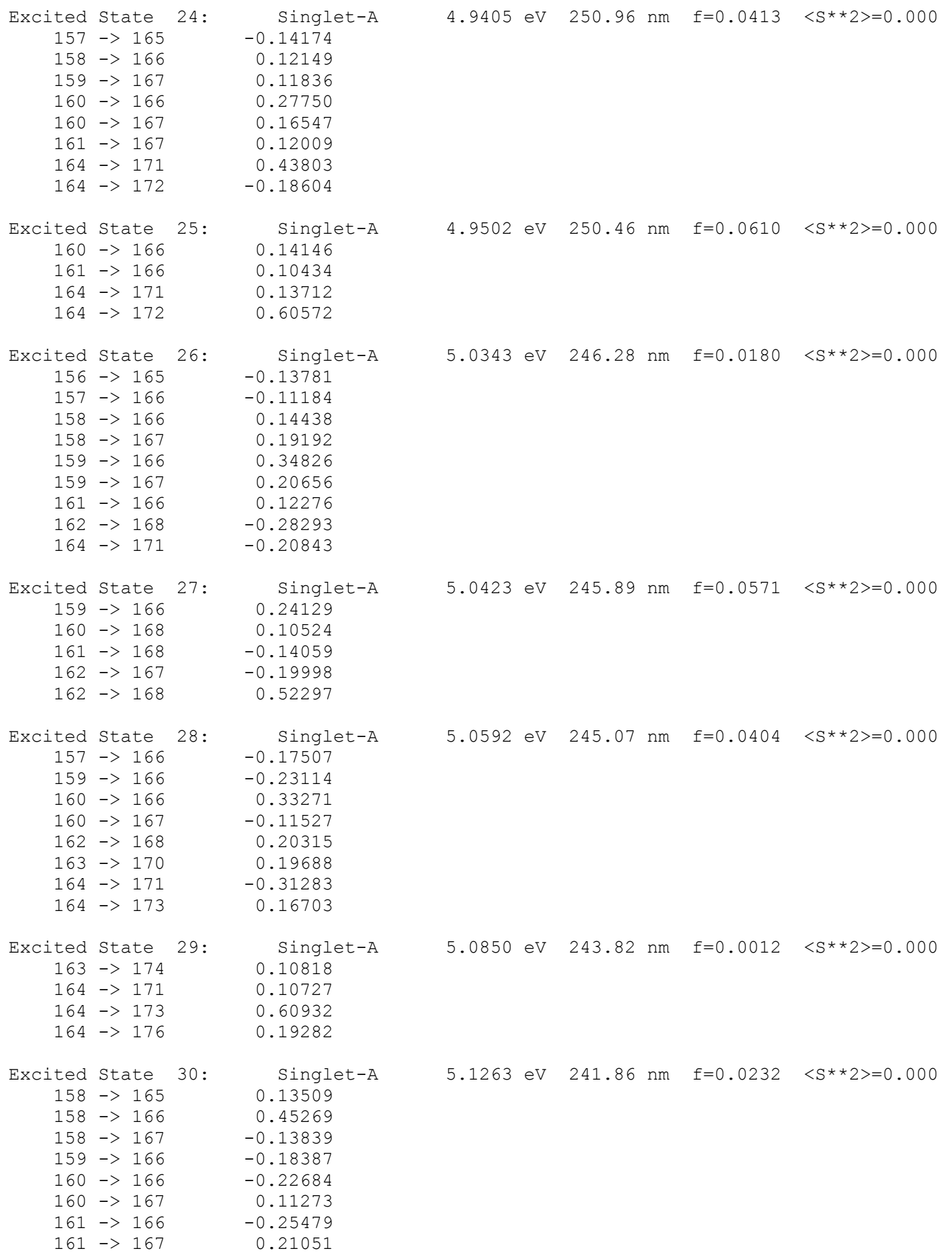




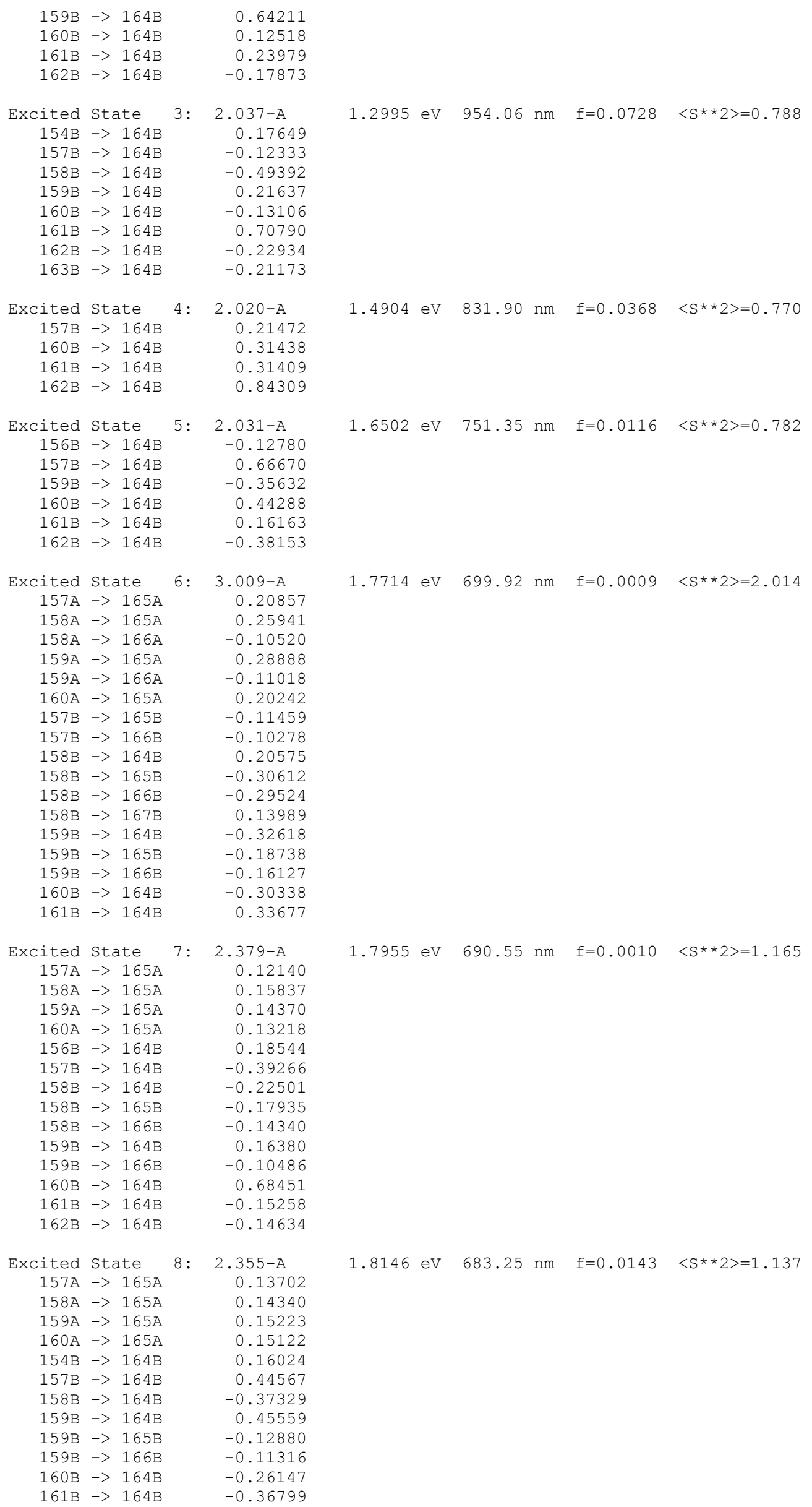




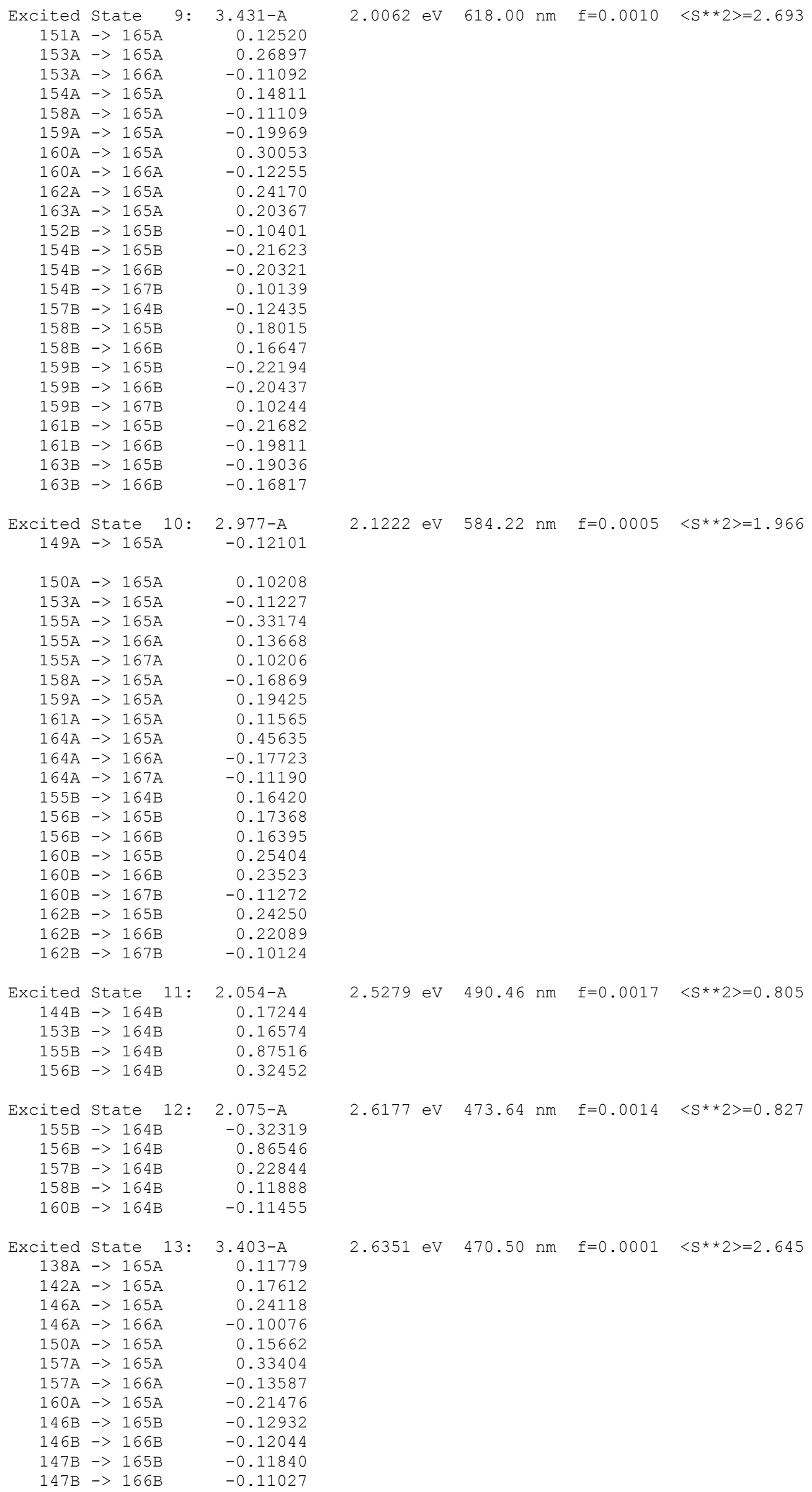




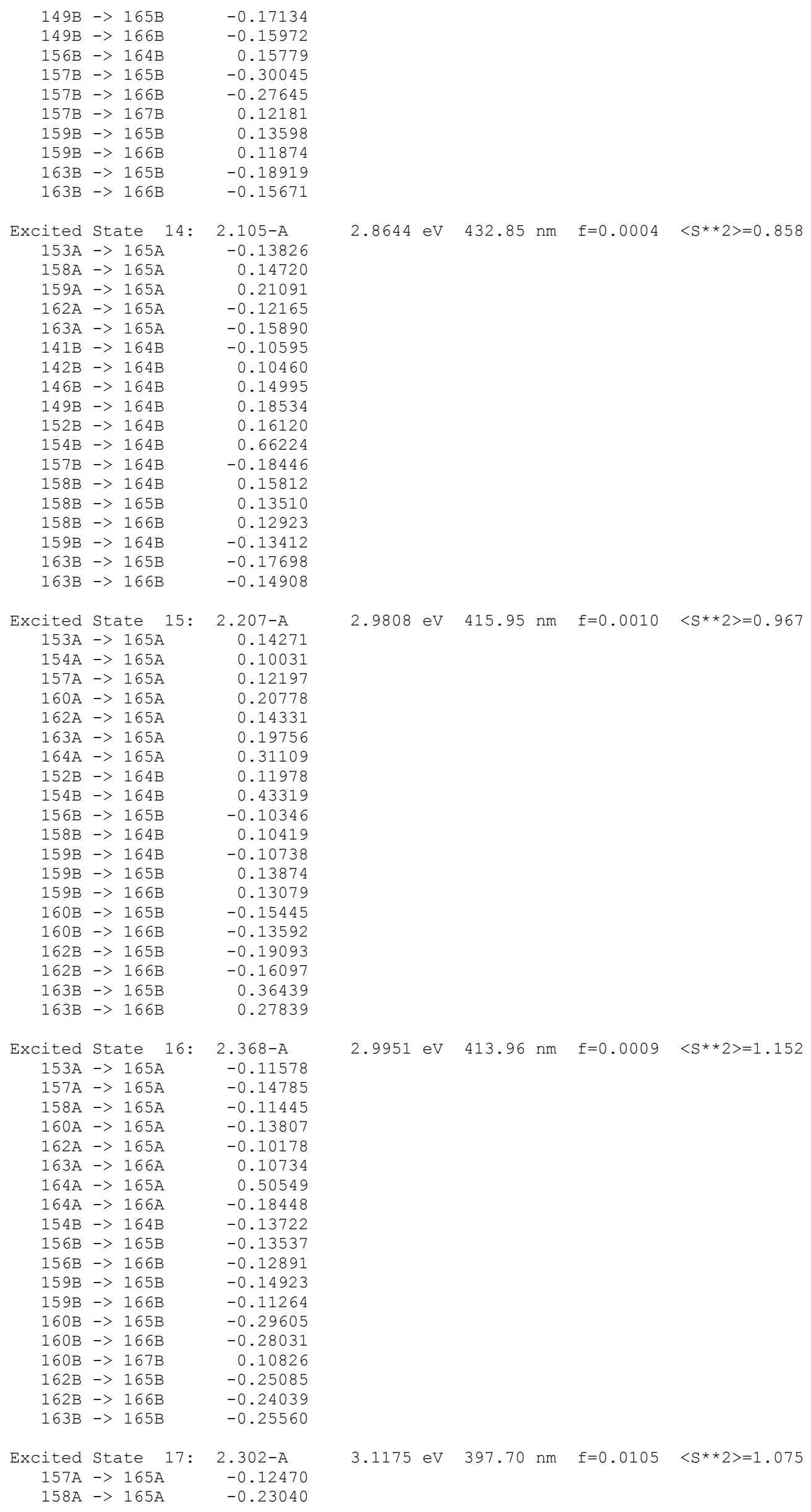




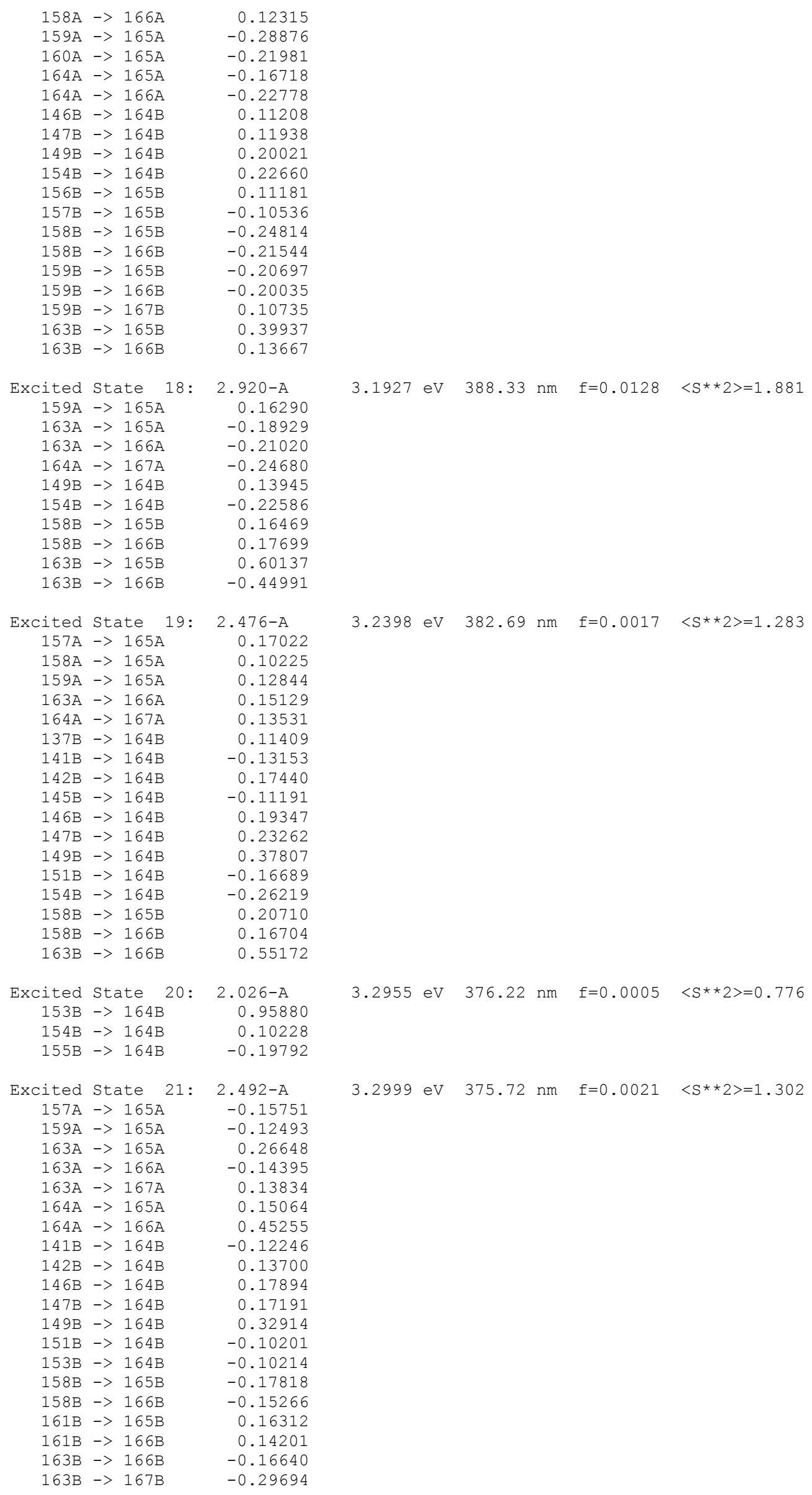




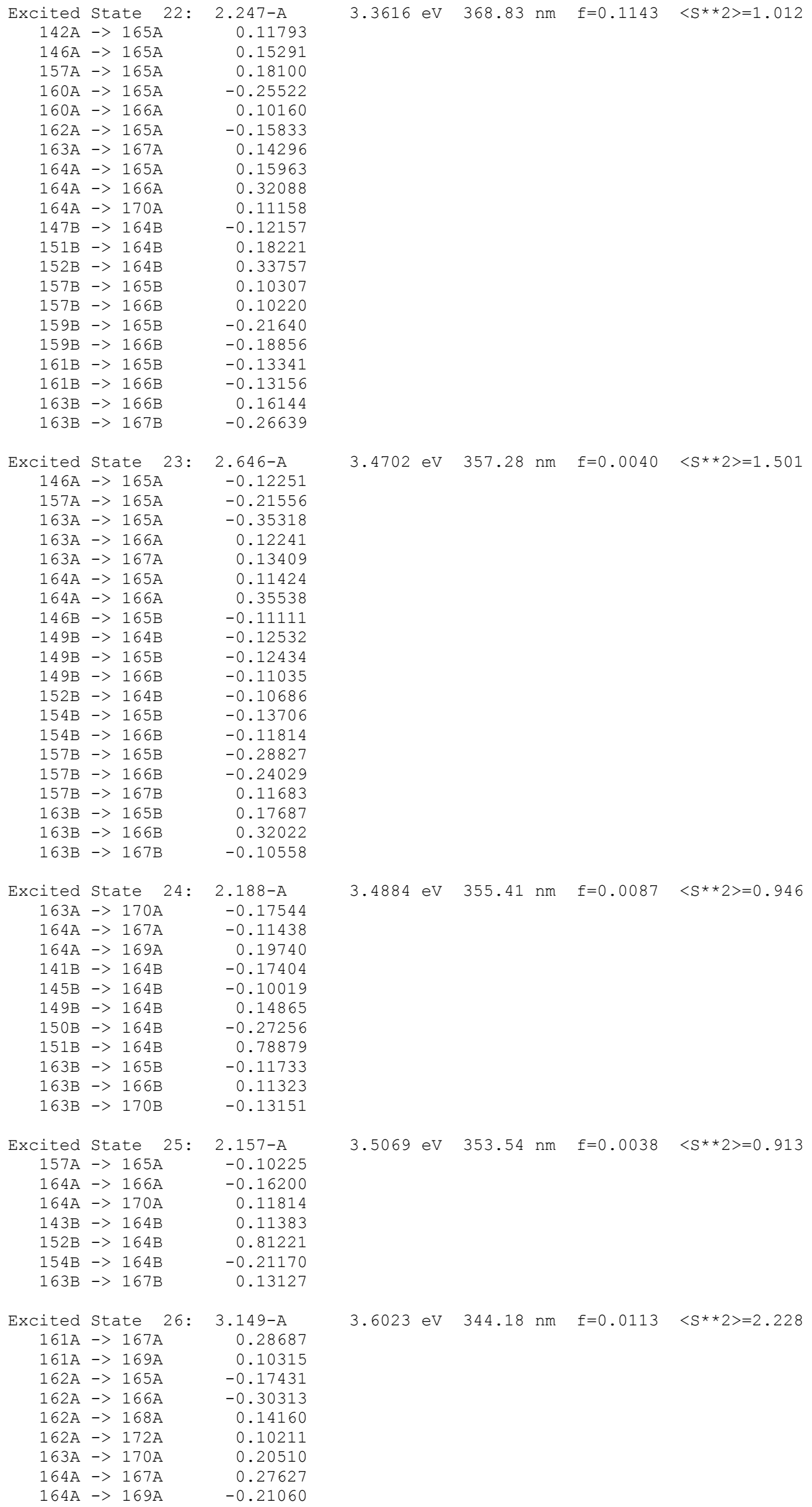




\begin{tabular}{|c|c|c|}
\hline $164 \mathrm{~A}$ & $->171 \mathrm{~A}$ & -0.10071 \\
\hline $150 \mathrm{~B}$ & $->164 \mathrm{~B}$ & -0.21168 \\
\hline $151 B$ & $->164 \mathrm{~B}$ & 0.18534 \\
\hline $160 \mathrm{~B}$ & $->167 \mathrm{~B}$ & -0.16315 \\
\hline $161 \mathrm{~B}$ & $->165 \mathrm{~B}$ & 0.26609 \\
\hline $161 \mathrm{~B}$ & $->166 \mathrm{~B}$ & -0.19326 \\
\hline $161 B$ & $->168 \mathrm{~B}$ & -0.11298 \\
\hline $162 B$ & $->166 \mathrm{~B}$ & 0.12758 \\
\hline $162 \mathrm{~B}$ & $->167 \mathrm{~B}$ & 0.19051 \\
\hline $163 B$ & $->165 \mathrm{~B}$ & 0.10433 \\
\hline $163 \mathrm{~B}$ & $->166 \mathrm{~B}$ & -0.15990 \\
\hline $163 B$ & $->170 \mathrm{~B}$ & 0.31286 \\
\hline Excited & state & $2.049-\mathrm{A}$ \\
\hline $146 \mathrm{~B}$ & $->164 \mathrm{~B}$ & 0.12567 \\
\hline $147 \mathrm{~B}$ & $->164 \mathrm{~B}$ & 0.11899 \\
\hline $150 B$ & $->164 \mathrm{~B}$ & 0.90112 \\
\hline $151 B$ & $->164 \mathrm{~B}$ & 0.31474 \\
\hline $152 B$ & $->164 \mathrm{~B}$ & -0.10393 \\
\hline Excited & State & $3.234-\mathrm{A}$ \\
\hline $161 \mathrm{~A}$ & $->165 \mathrm{~A}$ & 0.14505 \\
\hline $161 \mathrm{~A}$ & $->166 \mathrm{~A}$ & 0.28263 \\
\hline $161 \mathrm{~A}$ & $->168 \mathrm{~A}$ & -0.17797 \\
\hline $161 \mathrm{~A}$ & $->172 \mathrm{~A}$ & -0.12729 \\
\hline $162 \mathrm{~A}$ & $->165 \mathrm{~A}$ & -0.10225 \\
\hline $162 \mathrm{~A}$ & $->167 \mathrm{~A}$ & -0.29029 \\
\hline $163 \mathrm{~A}$ & $->169 A$ & -0.25658 \\
\hline $163 \mathrm{~A}$ & $->171 \mathrm{~A}$ & -0.14372 \\
\hline $164 \mathrm{~A}$ & $->165 \mathrm{~A}$ & -0.13761 \\
\hline $164 \mathrm{~A}$ & $->166 \mathrm{~A}$ & -0.22101 \\
\hline $164 \mathrm{~A}$ & $->168 \mathrm{~A}$ & 0.12506 \\
\hline $164 \mathrm{~A}$ & $->170 \mathrm{~A}$ & 0.30027 \\
\hline $160 \mathrm{~B}$ & $->165 \mathrm{~B}$ & -0.11008 \\
\hline $161 \mathrm{~B}$ & $->167 \mathrm{~B}$ & 0.25601 \\
\hline $162 \mathrm{~B}$ & $->165 \mathrm{~B}$ & 0.16755 \\
\hline $162 B$ & $->166 \mathrm{~B}$ & -0.22671 \\
\hline $162 \mathrm{~B}$ & $->168 \mathrm{~B}$ & -0.13784 \\
\hline $162 B$ & $->172 \mathrm{~B}$ & -0.10257 \\
\hline $163 \mathrm{~B}$ & $->169 \mathrm{~B}$ & 0.29590 \\
\hline $163 \mathrm{~B}$ & $->171 B$ & 0.21139 \\
\hline Excited & State & $2.915-\mathrm{A}$ \\
\hline $162 \mathrm{~A}$ & $->165 \mathrm{~A}$ & -0.10868 \\
\hline $163 \mathrm{~A}$ & $->165 \mathrm{~A}$ & 0.20543 \\
\hline $163 \mathrm{~A}$ & $->167 \mathrm{~A}$ & -0.19389 \\
\hline $164 \mathrm{~A}$ & $->165 \mathrm{~A}$ & 0.19336 \\
\hline $164 \mathrm{~A}$ & $->166 \mathrm{~A}$ & 0.36537 \\
\hline $164 \mathrm{~A}$ & $->168 \mathrm{~A}$ & 0.14883 \\
\hline $162 \mathrm{~B}$ & $->165 \mathrm{~B}$ & 0.13268 \\
\hline $162 \mathrm{~B}$ & $->166 \mathrm{~B}$ & -0.10460 \\
\hline $163 B$ & $->167 \mathrm{~B}$ & 0.76176 \\
\hline Excited & State 30 : & $2.361-A$ \\
\hline $157 \mathrm{~A}$ & $->165 \mathrm{~A}$ & -0.15166 \\
\hline $158 \mathrm{~A}$ & $->165 \mathrm{~A}$ & 0.13294 \\
\hline $159 \mathrm{~A}$ & $->165 \mathrm{~A}$ & 0.12450 \\
\hline $162 \mathrm{~A}$ & $->165 \mathrm{~A}$ & -0.13901 \\
\hline $162 \mathrm{~A}$ & $->166 \mathrm{~A}$ & 0.12325 \\
\hline $163 \mathrm{~A}$ & $->165 \mathrm{~A}$ & 0.63135 \\
\hline $163 \mathrm{~A}$ & $->166 \mathrm{~A}$ & 0.19188 \\
\hline $164 \mathrm{~A}$ & $->166 \mathrm{~A}$ & -0.18188 \\
\hline $164 \mathrm{~A}$ & $->167 \mathrm{~A}$ & 0.27200 \\
\hline $145 B$ & $->164 \mathrm{~B}$ & 0.11429 \\
\hline $149 B$ & $->164 \mathrm{~B}$ & -0.13845 \\
\hline $161 \mathrm{~B}$ & $->165 \mathrm{~B}$ & -0.10719 \\
\hline $163 \mathrm{~B}$ & $->165 \mathrm{~B}$ & 0.24434 \\
\hline $163 B$ & $->166 \mathrm{~B}$ & -0.12696 \\
\hline $163 B$ & $->168 \mathrm{~B}$ & 0.1346 \\
\hline
\end{tabular}

\section{$1_{\mathrm{Py}^{+}}^{+}$}

Excited State 1: 2.036-A $156 \mathrm{~B}->185 \mathrm{~B} \quad-0.12741$
$3.6136 \mathrm{eV} \quad 343.11 \mathrm{~nm} \quad \mathrm{f}=0.0013 \quad<\mathrm{S} * 2>=0.799$

$3.6654 \mathrm{eV} \quad 338.26 \mathrm{~nm} \quad \mathrm{f}=0.0455 \quad\langle\mathrm{~S} * * 2>=2.365$

$3.7170 \mathrm{eV} \quad 333.56 \mathrm{~nm} \quad \mathrm{f}=0.0177 \quad\langle\mathrm{~S} * * 2>=1.874$

$3.7562 \mathrm{eV} \quad 330.08 \mathrm{~nm} \quad \mathrm{f}=0.0877 \quad<\mathrm{S} * * 2>=1.143$ 


$$
\begin{array}{lr}
158 \mathrm{~B}->185 \mathrm{~B} & -0.13973 \\
164 \mathrm{~B}->185 \mathrm{~B} & -0.11267 \\
168 \mathrm{~B}->185 \mathrm{~B} & -0.18952 \\
173 \mathrm{~B}->185 \mathrm{~B} & 0.17604 \\
174 \mathrm{~B}->185 \mathrm{~B} & -0.10966 \\
177 \mathrm{~B}->185 \mathrm{~B} & -0.17224 \\
179 \mathrm{~B}->185 \mathrm{~B} & -0.24941 \\
184 \mathrm{~B}->185 \mathrm{~B} & 0.81646
\end{array}
$$

This state for optimization and/or second-order correction.

Total Energy, E (TD-HF/TD-KS) = -3488.21253371

Copying the excited state density for this state as the 1-particle RhocI density.

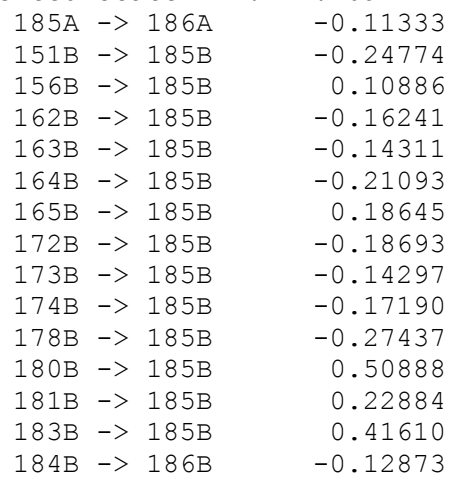




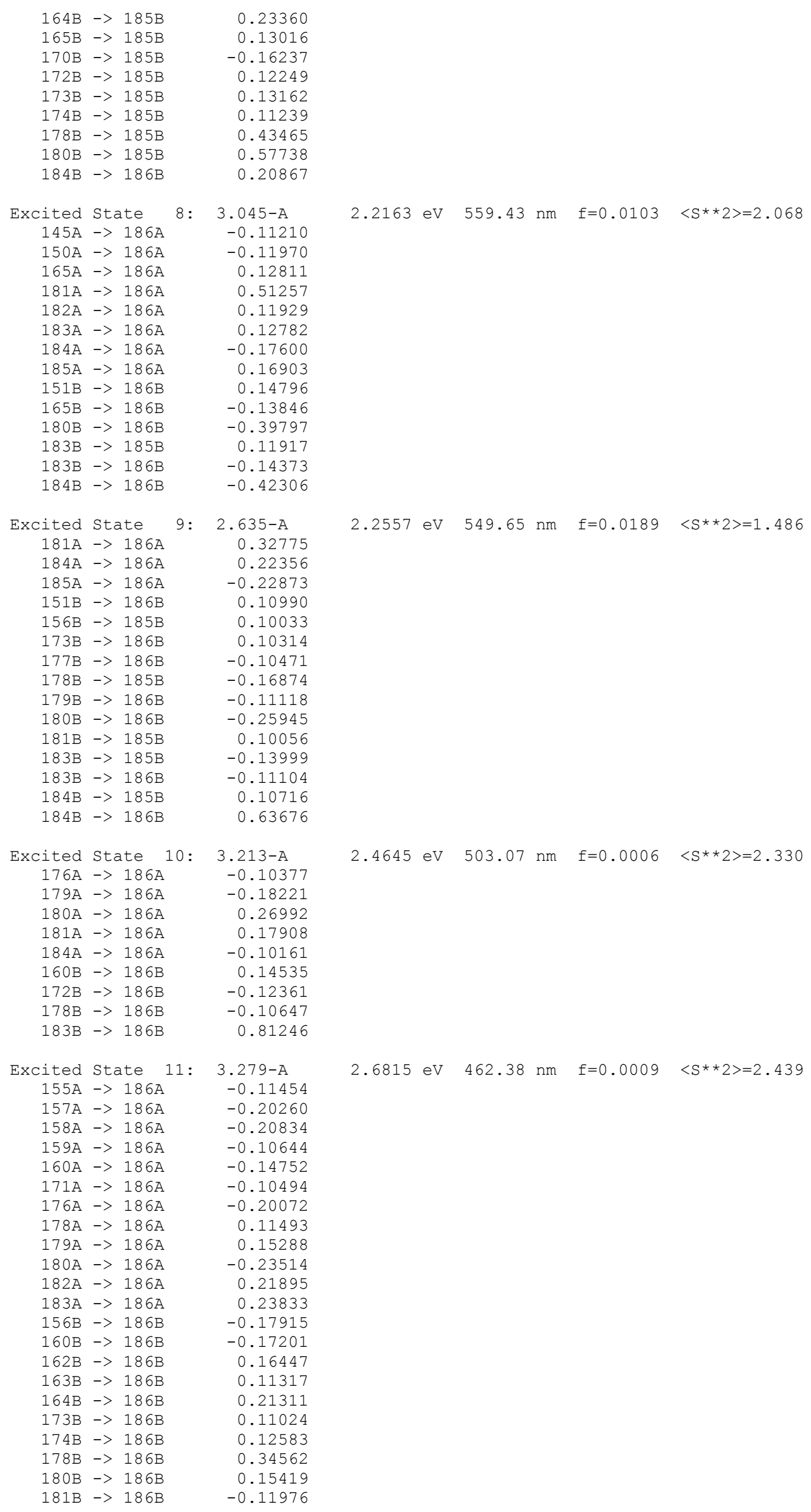




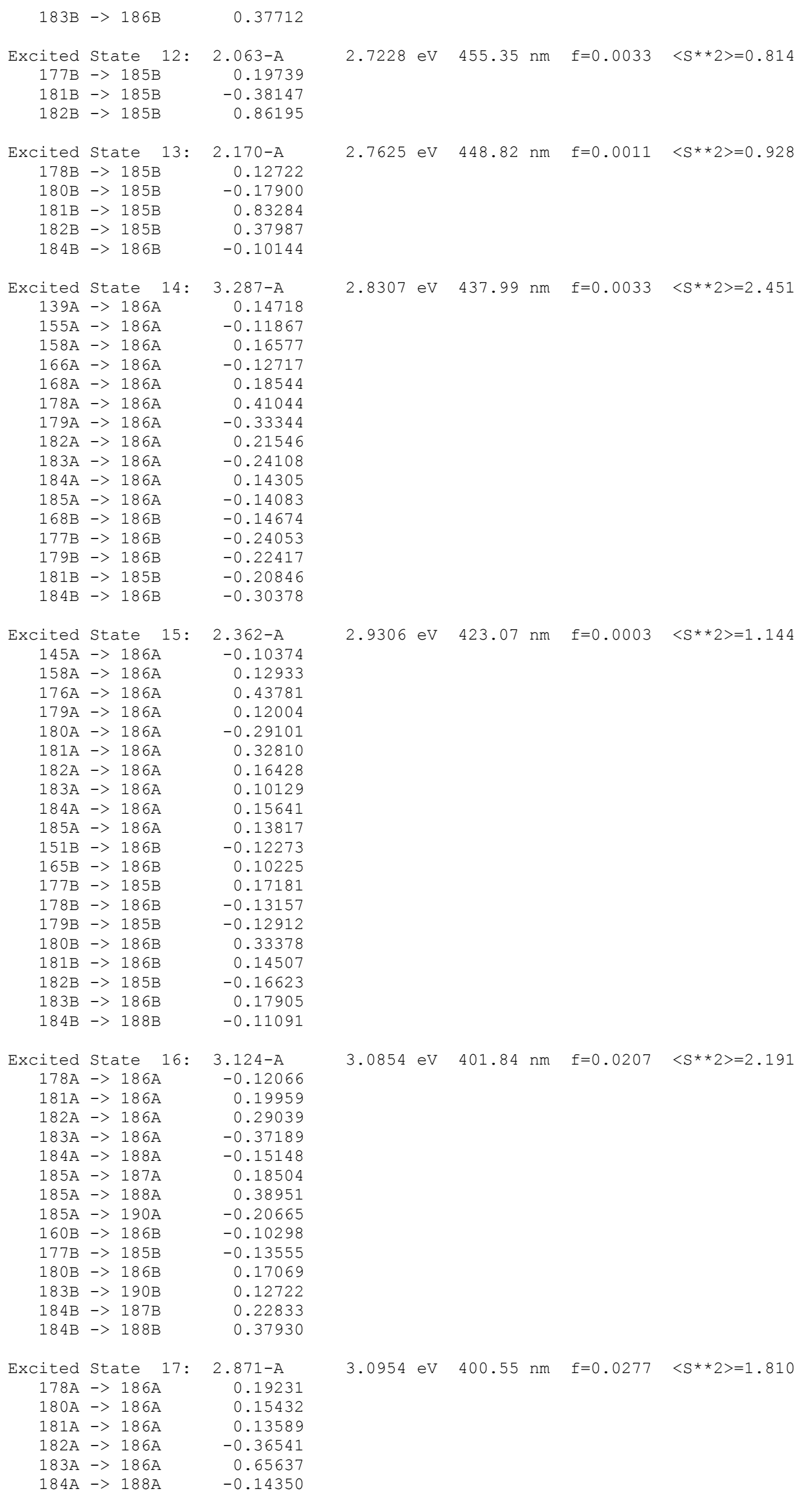




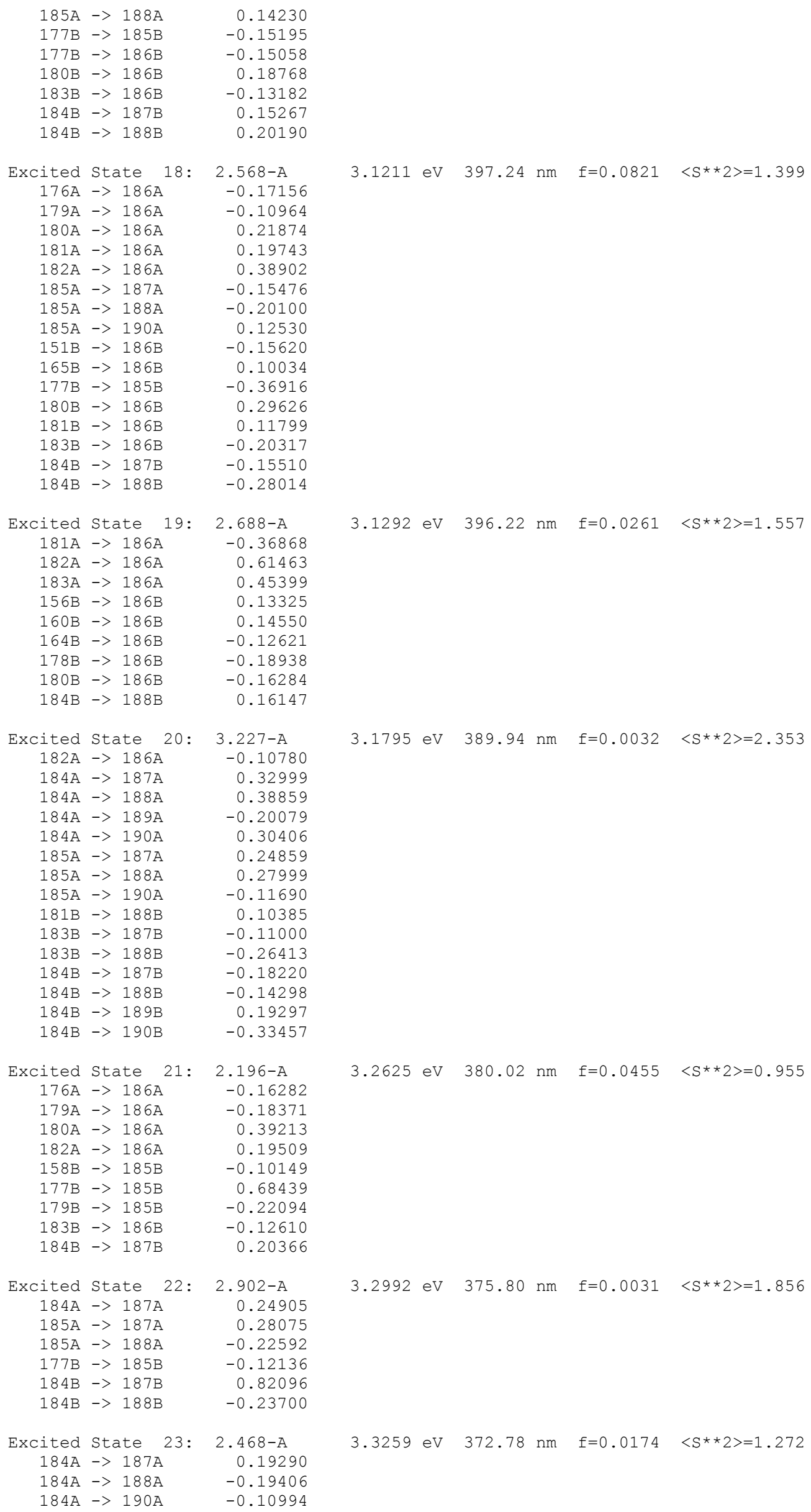




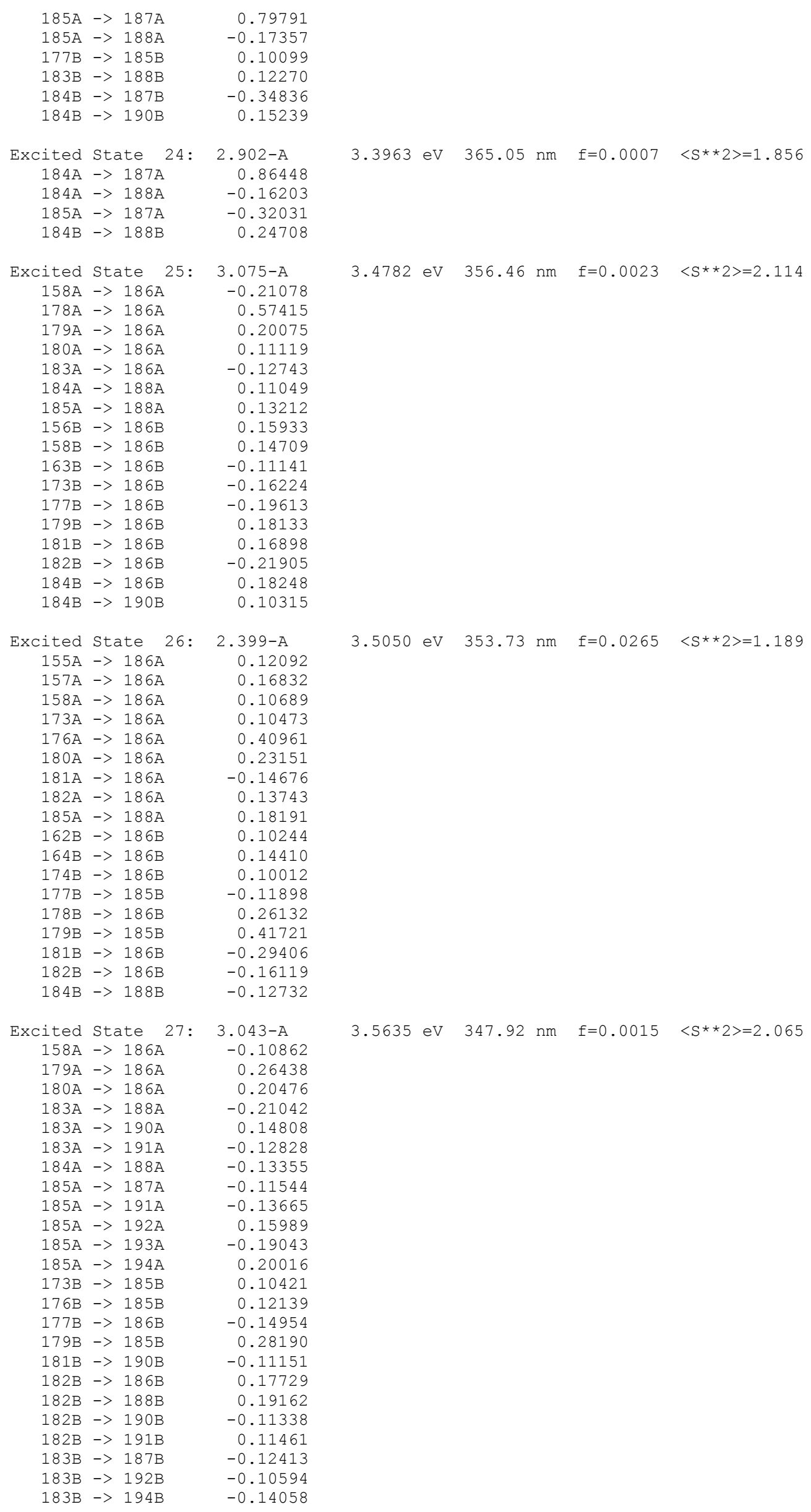




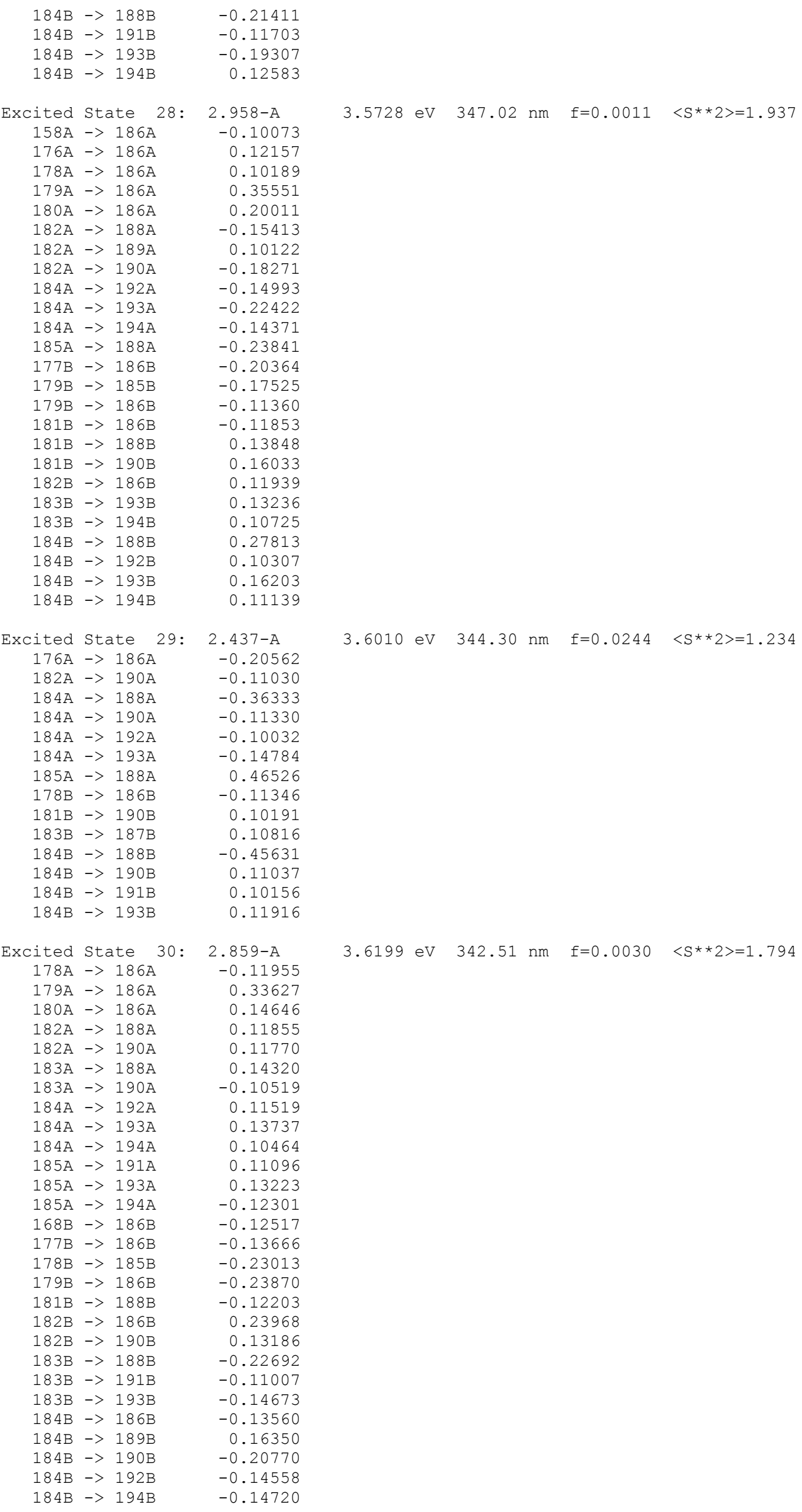




\section{$1_{\mathrm{Py2}^{+}}$}

\begin{tabular}{cr} 
Excited State $1:$ & \multicolumn{1}{l}{$2.032-\mathrm{A}$} \\
$166 \mathrm{~B}->206 \mathrm{~B}$ & -0.10406 \\
$171 \mathrm{~B}->206 \mathrm{~B}$ & -0.13414 \\
$174 \mathrm{~B}->206 \mathrm{~B}$ & 0.19056 \\
$175 \mathrm{~B}->206 \mathrm{~B}$ & -0.12172 \\
$176 \mathrm{~B}->206 \mathrm{~B}$ & 0.11892 \\
$177 \mathrm{~B}->206 \mathrm{~B}$ & -0.17210 \\
$181 \mathrm{~B}->206 \mathrm{~B}$ & -0.20038 \\
$183 \mathrm{~B}->206 \mathrm{~B}$ & 0.11732 \\
$186 \mathrm{~B}->206 \mathrm{~B}$ & -0.12146 \\
$191 \mathrm{~B}->206 \mathrm{~B}$ & 0.17688 \\
$193 \mathrm{~B}->206 \mathrm{~B}$ & -0.19305 \\
$194 \mathrm{~B}->206 \mathrm{~B}$ & 0.19514 \\
$196 \mathrm{~B}->206 \mathrm{~B}$ & 0.12239 \\
$198 \mathrm{~B}->206 \mathrm{~B}$ & 0.11294 \\
$200 \mathrm{~B}->206 \mathrm{~B}$ & -0.18934 \\
$205 \mathrm{~B}->206 \mathrm{~B}$ & 0.75150
\end{tabular}

This state for optimization and/or second-order correction.

Total Energy, E(TD-HF/TD-KS) = -3736.59143861

Copying the excited state density for this state as the 1-particle RhocI density.

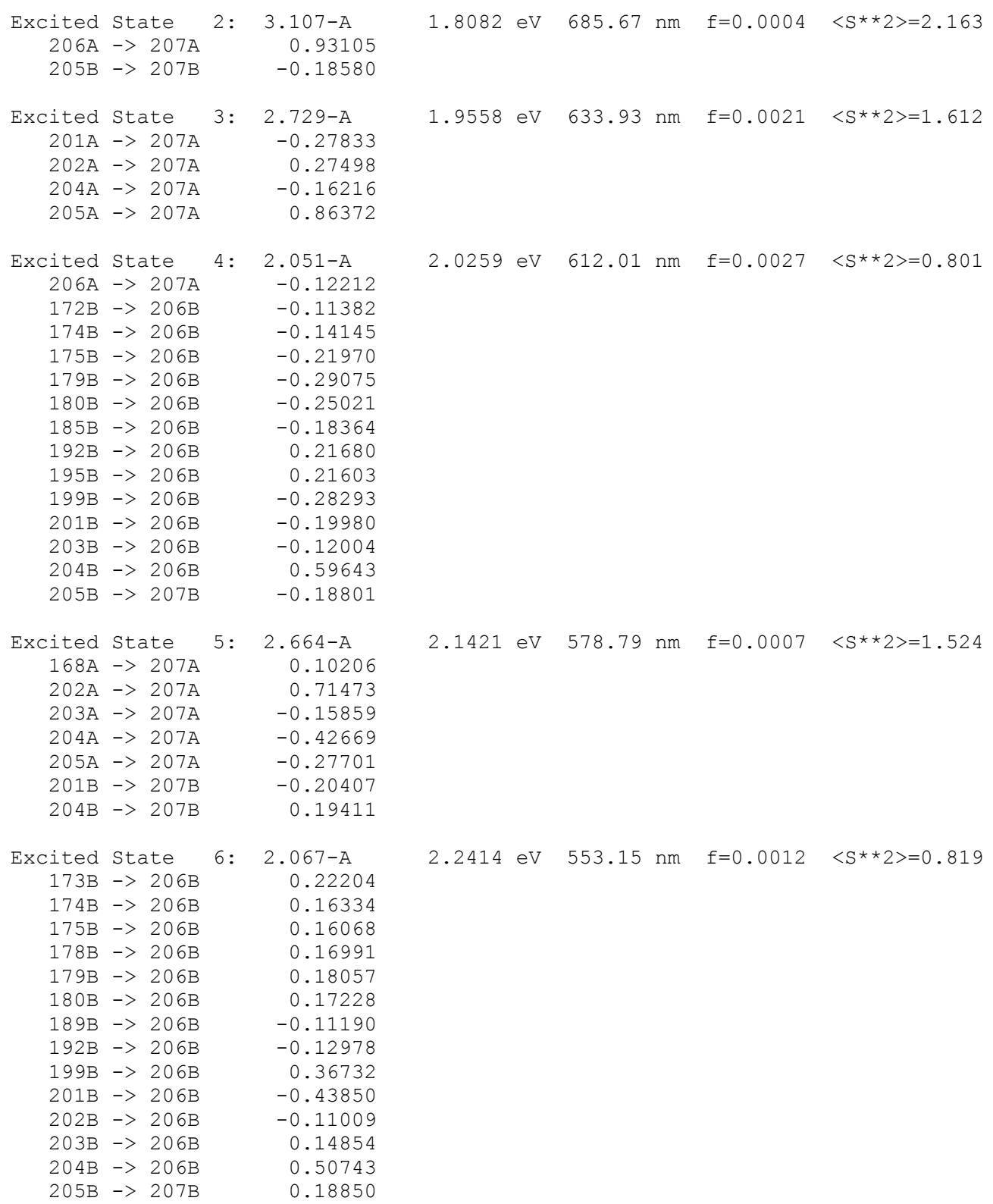




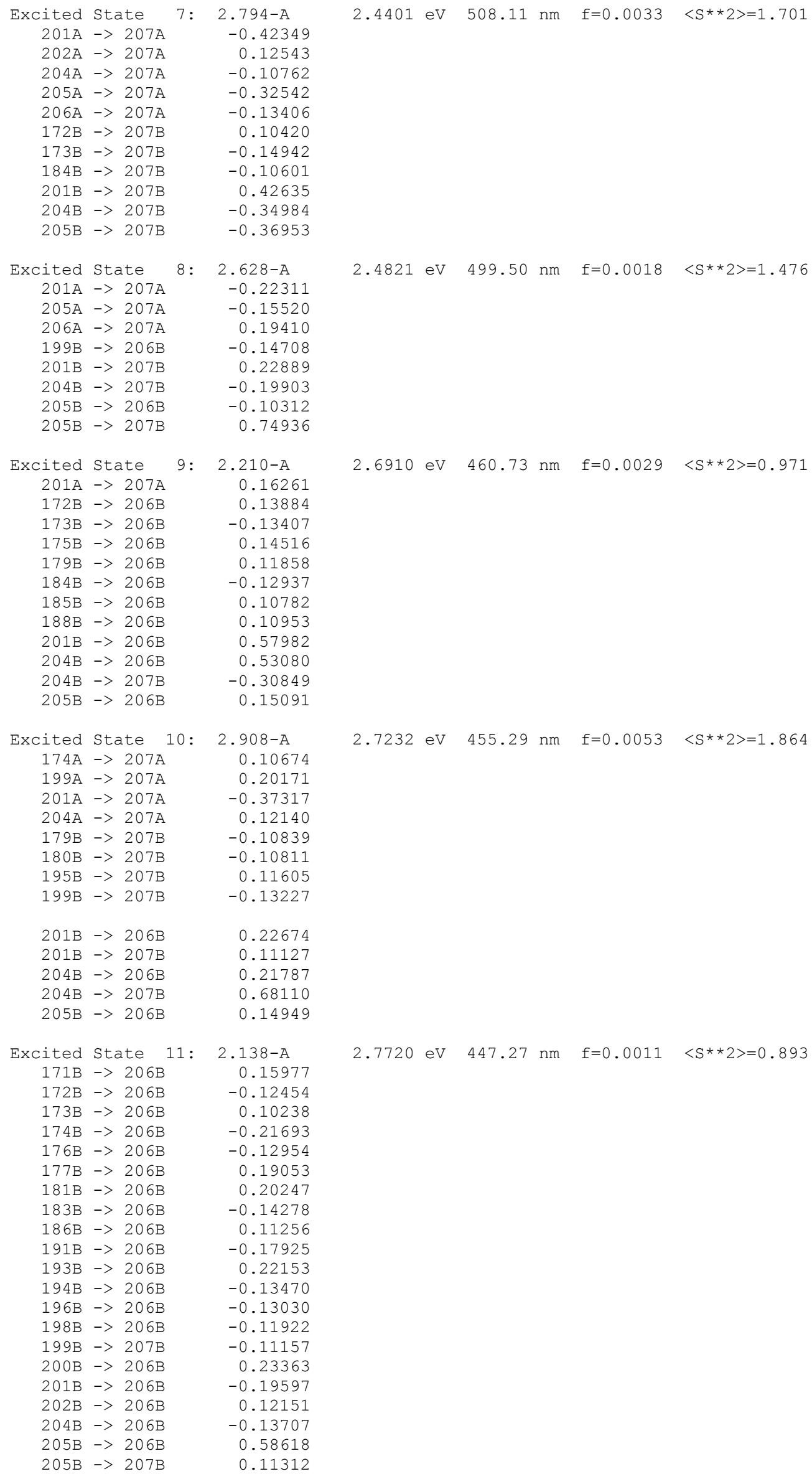




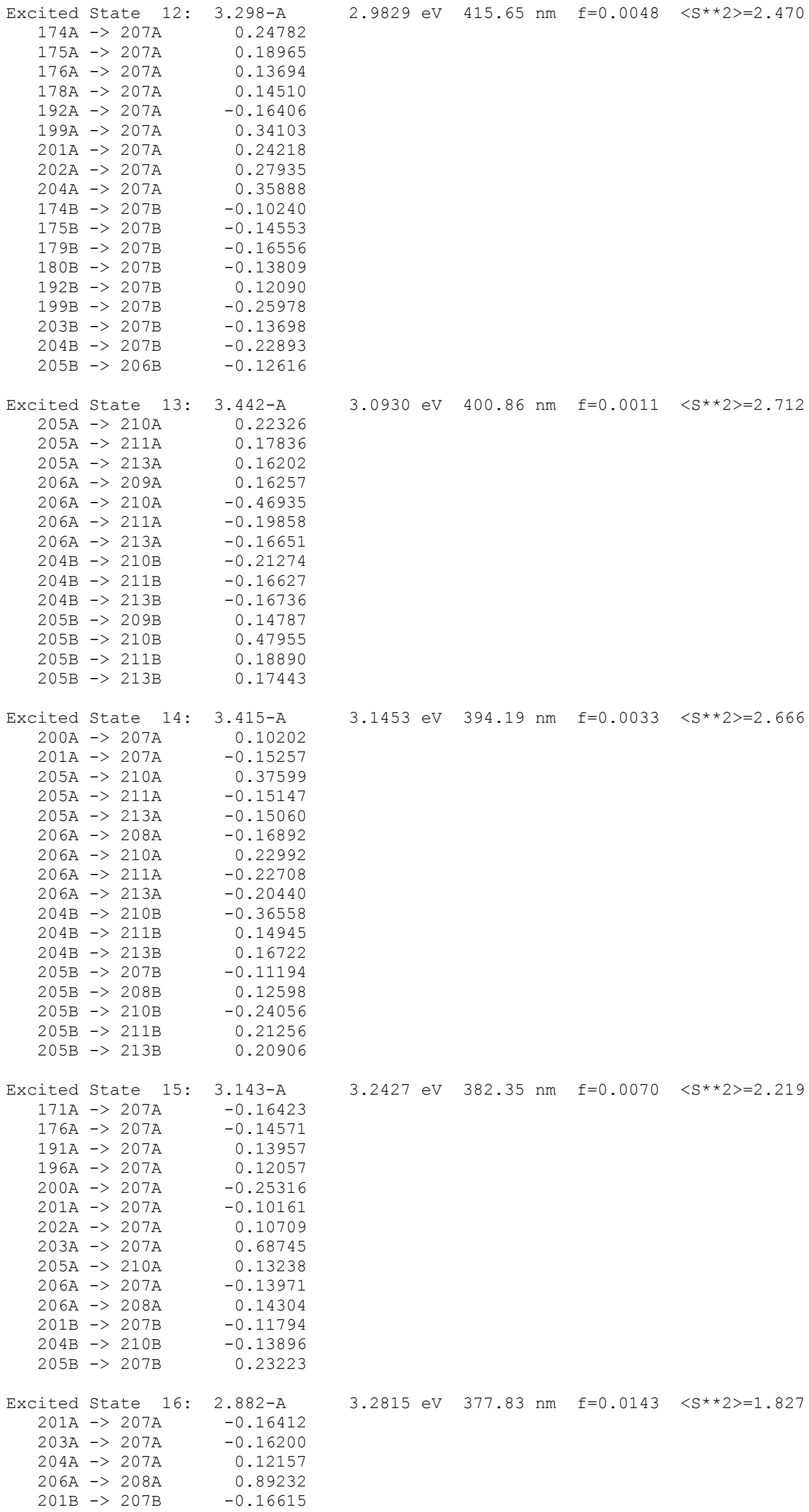




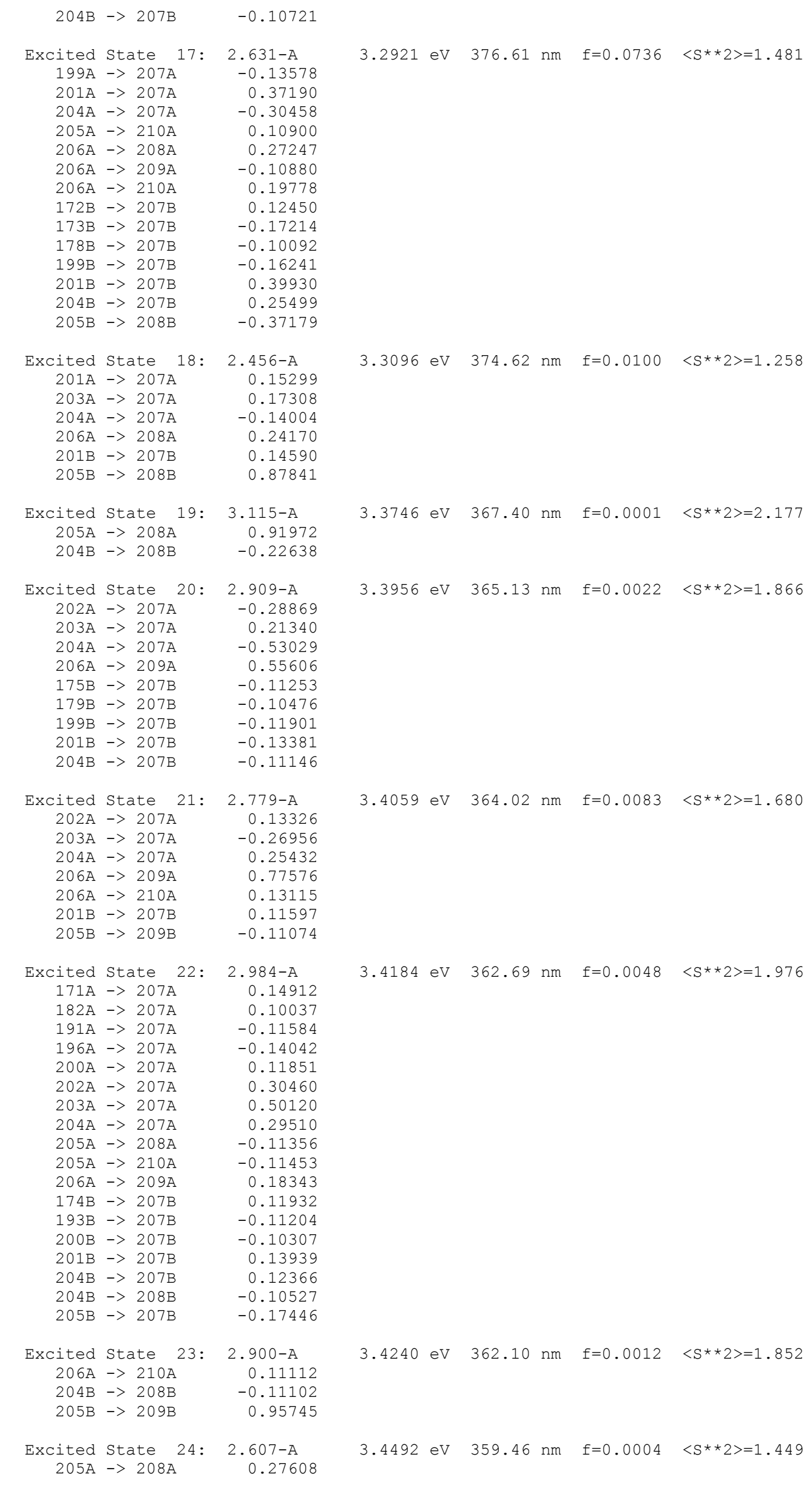




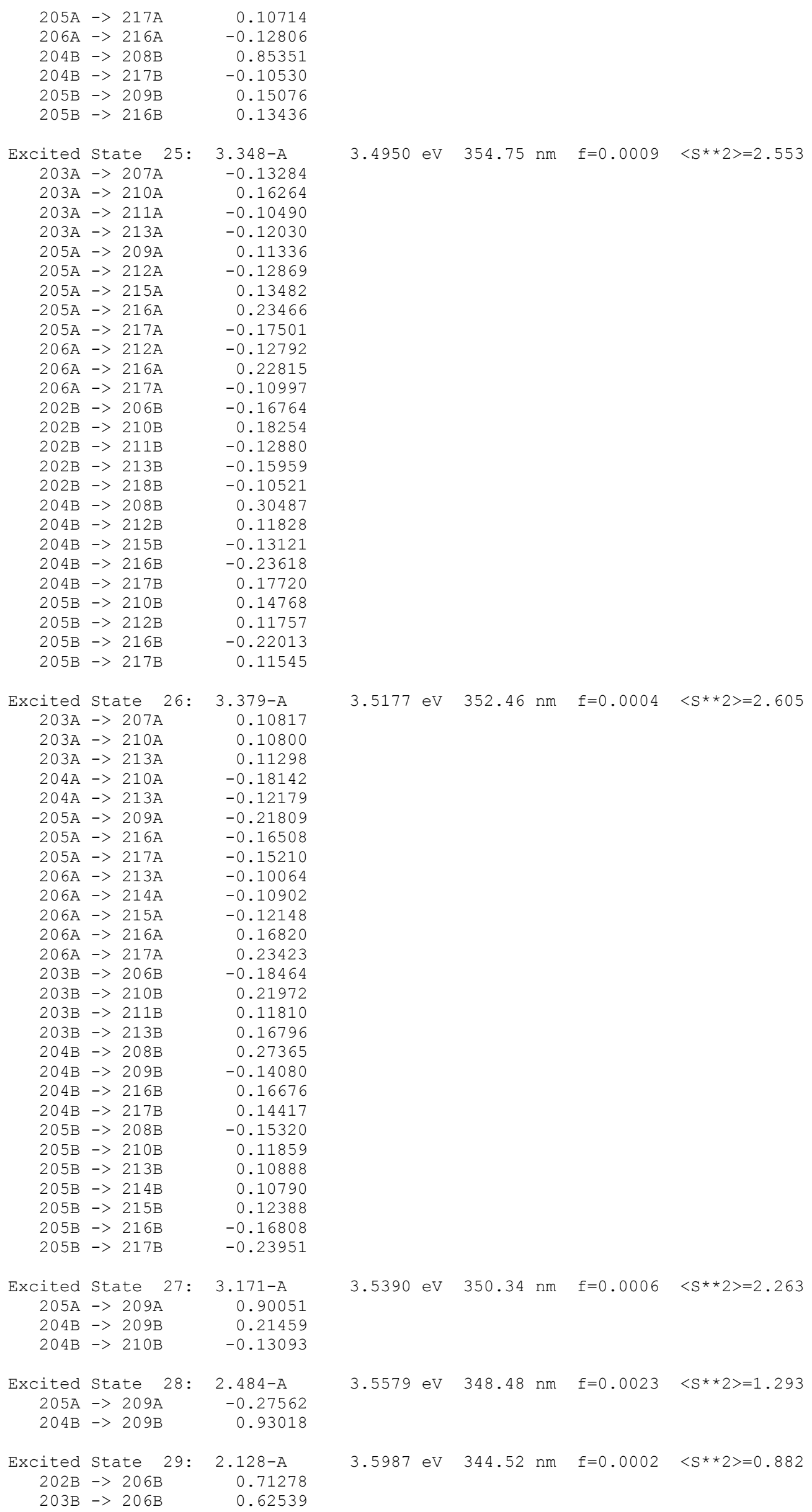




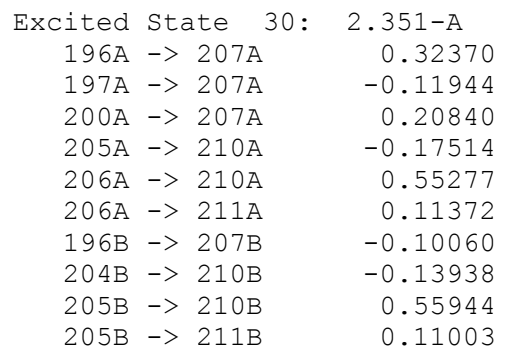

$205 B->211 B \quad 0.11003$

\section{${ }^{3} 1^{2+}$}

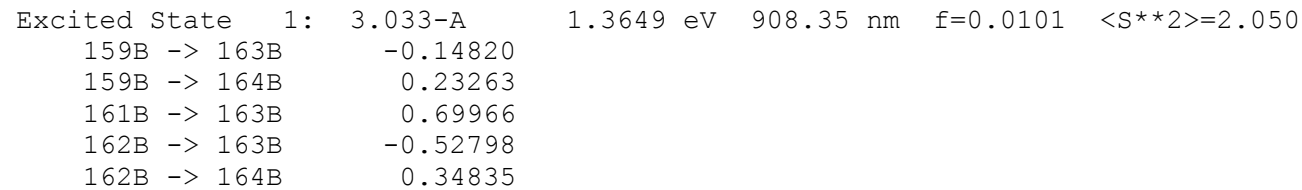

This state for optimization and/or second-order correction.

Total Energy, E (TD-HF/TD-KS $)=-3239.58754256$

Copying the excited state density for this state as the 1-particle RhocI density.

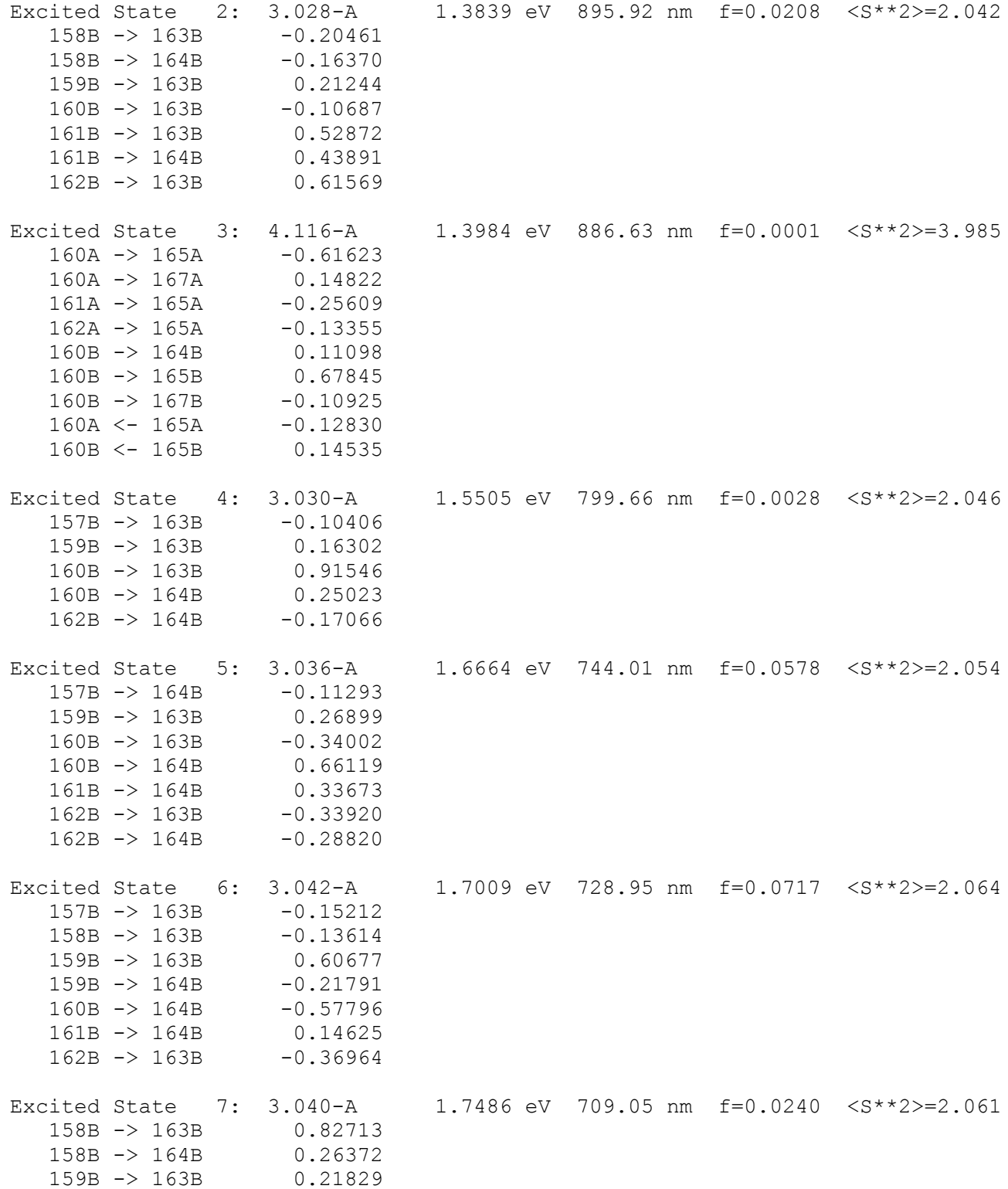




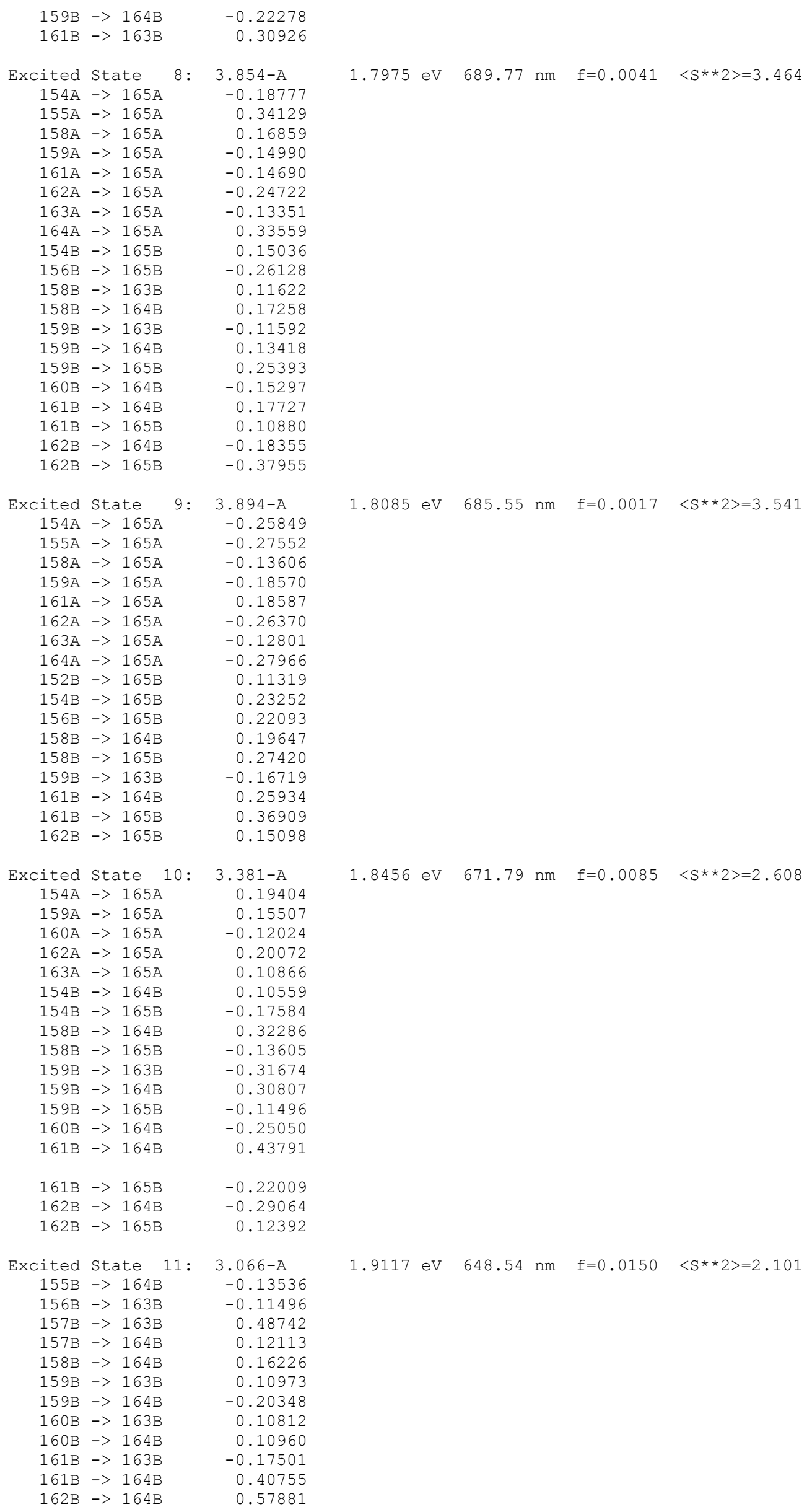




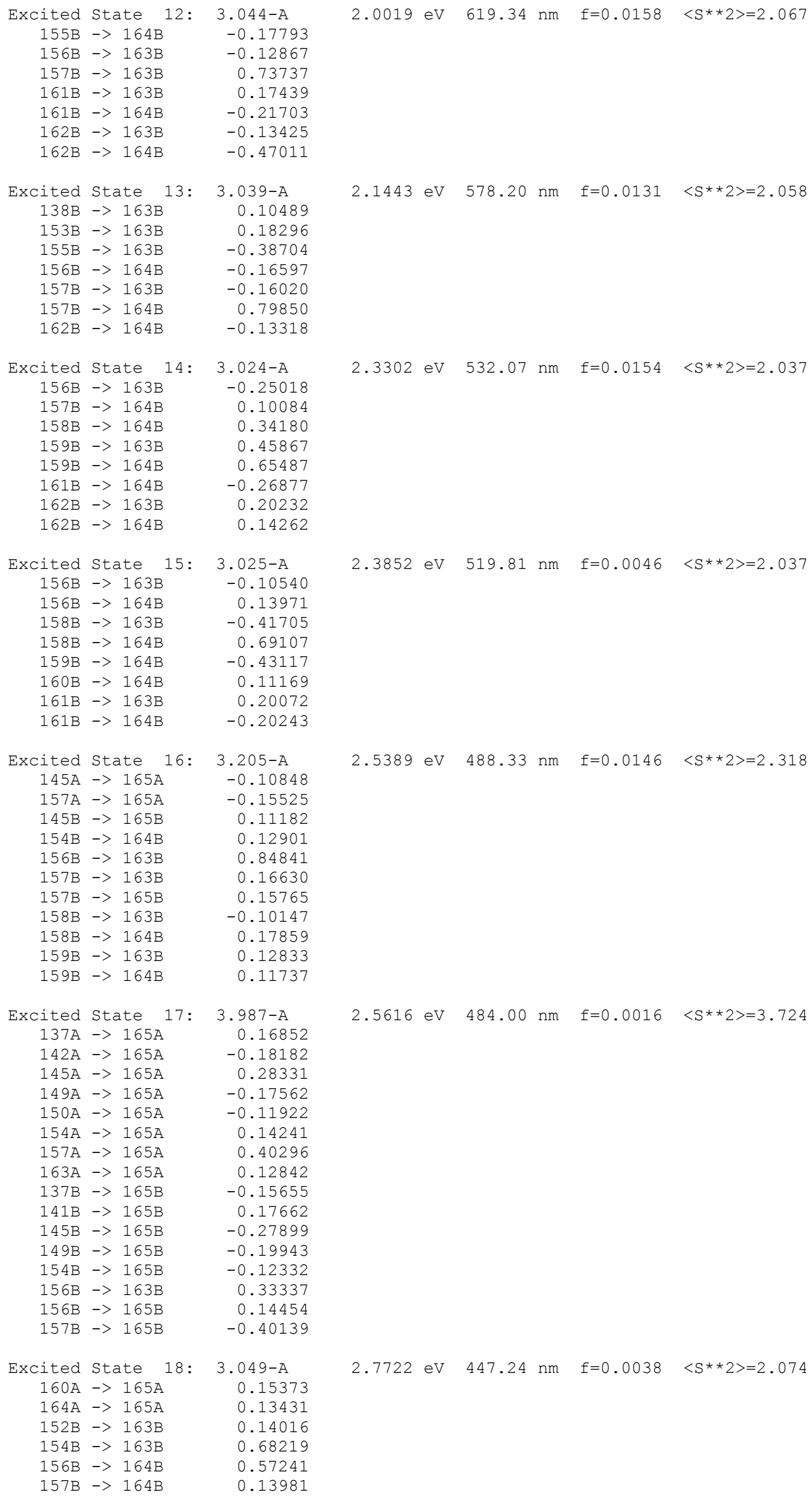




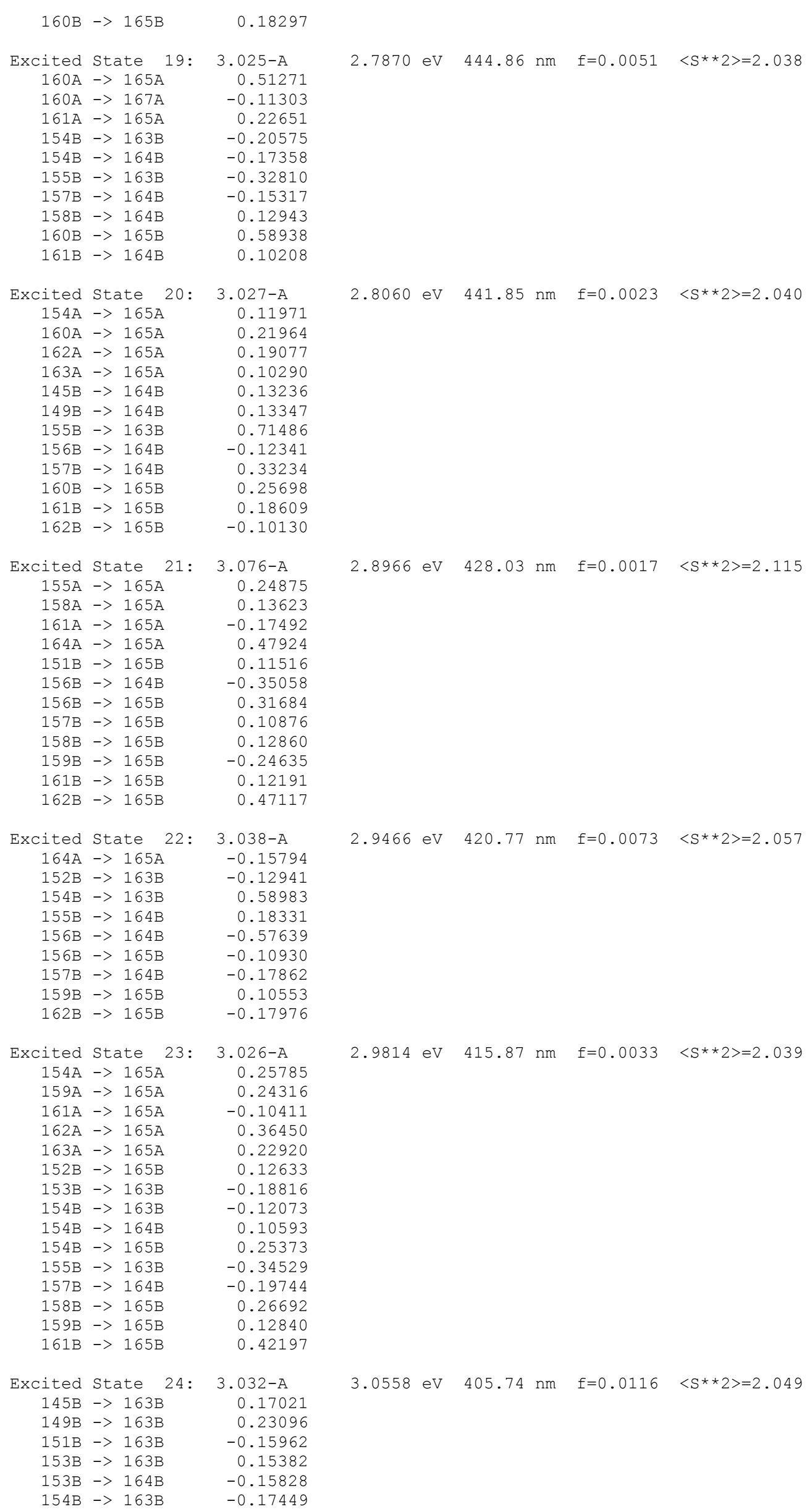

$160 \mathrm{~B}->165 \mathrm{~B}$

0.18297

Excited State 19:

160A $->165 A$

$160 \mathrm{~A} \rightarrow 167 \mathrm{~A}$

$161 \mathrm{~A}->165 \mathrm{~A}$

$154 \mathrm{~B}->163 \mathrm{~B}$

$154 \mathrm{~B}->164 \mathrm{~B}$

$155 \mathrm{~B}->163 \mathrm{~B}$

$157 \mathrm{~B}->164 \mathrm{~B}$

$158 \mathrm{~B}->164 \mathrm{~B}$

$160 \mathrm{~B}->165 \mathrm{~B}$

$161 \mathrm{~B}->164 \mathrm{~B}$

Excited State 20:

$154 \mathrm{~A} \rightarrow 165 \mathrm{~A}$

$160 \mathrm{~A} \rightarrow 165 \mathrm{~A}$

$162 \mathrm{~A} \rightarrow 165 \mathrm{~A}$

$163 \mathrm{~A} \rightarrow 165 \mathrm{~A}$

$145 \mathrm{~B} \rightarrow 164 \mathrm{~B}$

$149 \mathrm{~B} \rightarrow 164 \mathrm{~B}$

$155 \mathrm{~B} \rightarrow>163 \mathrm{~B}$

$156 \mathrm{~B} \rightarrow 164 \mathrm{~B}$

$157 \mathrm{~B}->164 \mathrm{~B}$

$160 \mathrm{~B}->165 \mathrm{~B}$

$161 \mathrm{~B}->165 \mathrm{~B}$

$162 \mathrm{~B}->165 \mathrm{~B}$

Excited State 21:

$155 \mathrm{~A} \rightarrow 165 \mathrm{~A}$

$158 \mathrm{~A} \rightarrow 165 \mathrm{~A}$

$161 \mathrm{~A} \rightarrow 165 \mathrm{~A}$

$164 \mathrm{~A}->165 \mathrm{~A}$

$151 \mathrm{~B} \rightarrow 165 \mathrm{~B}$

$156 \mathrm{~B}->164 \mathrm{~B}$

$156 \mathrm{~B}->165 \mathrm{~B}$

$157 \mathrm{~B} \rightarrow 165 \mathrm{~B}$

$158 \mathrm{~B} \rightarrow>165 \mathrm{~B}$

$159 \mathrm{~B} \rightarrow 165 \mathrm{~B}$

$161 \mathrm{~B} \rightarrow>165 \mathrm{~B}$

$162 \mathrm{~B}->165 \mathrm{~B}$

Excited State 22:

$164 \mathrm{~A}->165 \mathrm{~A}$

$152 \mathrm{~B} \rightarrow 163 \mathrm{~B}$

$154 \mathrm{~B} \rightarrow 163 \mathrm{~B}$

$155 \mathrm{~B}->164 \mathrm{~B}$

$156 \mathrm{~B} \rightarrow 164 \mathrm{~B}$

$156 \mathrm{~B} \rightarrow 165 \mathrm{~B}$

$157 \mathrm{~B}->164 \mathrm{~B}$

$159 \mathrm{~B}->165 \mathrm{~B}$

$162 \mathrm{~B}->165 \mathrm{~B}$

Excited State 23:

$154 \mathrm{~A}->165 \mathrm{~A}$

$159 \mathrm{~A} \rightarrow 165 \mathrm{~A}$

$161 \mathrm{~A} \rightarrow 165 \mathrm{~A}$

$162 \mathrm{~A} \rightarrow 165 \mathrm{~A}$

$163 \mathrm{~A}->165 \mathrm{~A}$

$152 \mathrm{~B}->165 \mathrm{~B}$

$153 \mathrm{~B}->163 \mathrm{~B}$

$154 \mathrm{~B}->163 \mathrm{~B}$

$154 \mathrm{~B}->164 \mathrm{~B}$

$154 \mathrm{~B}->165 \mathrm{~B}$

$155 \mathrm{~B}->163 \mathrm{~B}$

$157 \mathrm{~B} \rightarrow 164 \mathrm{~B}$

$158 \mathrm{~B}->165 \mathrm{~B}$

$159 \mathrm{~B}->165 \mathrm{~B}$

$161 \mathrm{~B} \rightarrow 165 \mathrm{~B}$

Excited State 24:

$145 \mathrm{~B}->163 \mathrm{~B}$

$149 \mathrm{~B}->163 \mathrm{~B}$

$151 \mathrm{~B}->163 \mathrm{~B}$

$153 \mathrm{~B}->163 \mathrm{~B}$

$153 \mathrm{~B}->164 \mathrm{~B}$

$154 \mathrm{~B}->163 \mathrm{~B}$

3. $025-\mathrm{A}$

0.51271

$-0.11303$

0.22651

$-0.20575$

$-0.17358$

$-0.32810$

$-0.15317$

0.12943

0.58938

0.10208

3. $027-\mathrm{A}$

0.11971

0.21964

0.19077

0.10290

0.13236

0.13347

0.71486

$-0.12341$

0.33234

0.25698

0.18609

$-0.10130$

$3.076-\mathrm{A}$

0.24875

0.13623

$-0.17492$

0.47924

0.11516

$-0.35058$

0.31684

0.10876

0.12860

$-0.24635$

0.12191

0.47117

3.038-A

$-0.15794$

$-0.12941$

0.58983

0.18331

$-0.57639$

$-0.10930$

$-0.17862$

0.10553

$-0.17976$

$3.026-\mathrm{A}$ 0.25785

0.24316

$-0.10411$

0.36450

0.22920

0.12633

$-0.18816$

$-0.12073$

0.10593

0.25373

$-0.34529$

$-0.19744$

0.26692

0.12840

0.42197

3. $032-\mathrm{A}$

0.17021

0.23096

$-0.15962$

0.15382

$-0.15828$

$-0.17449$

$2.7870 \mathrm{eV} \quad 444.86 \mathrm{~nm} \quad \mathrm{f}=0.0051 \quad<\mathrm{S} * * 2>=2.038$

$2.8060 \mathrm{eV} \quad 441.85 \mathrm{~nm} \quad \mathrm{f}=0.0023 \quad<\mathrm{S} * * 2>=2.040$

$2.8966 \mathrm{eV} \quad 428.03 \mathrm{~nm} \quad \mathrm{f}=0.0017 \quad<\mathrm{S} * * 2>=2.115$

$2.9466 \mathrm{eV} \quad 420.77 \mathrm{~nm} \quad \mathrm{f}=0.0073 \quad<\mathrm{S} * \star 2>=2.057$

$2.9814 \mathrm{eV} \quad 415.87 \mathrm{~nm} \quad \mathrm{f}=0.0033 \quad<* * 2>=2.039$ 


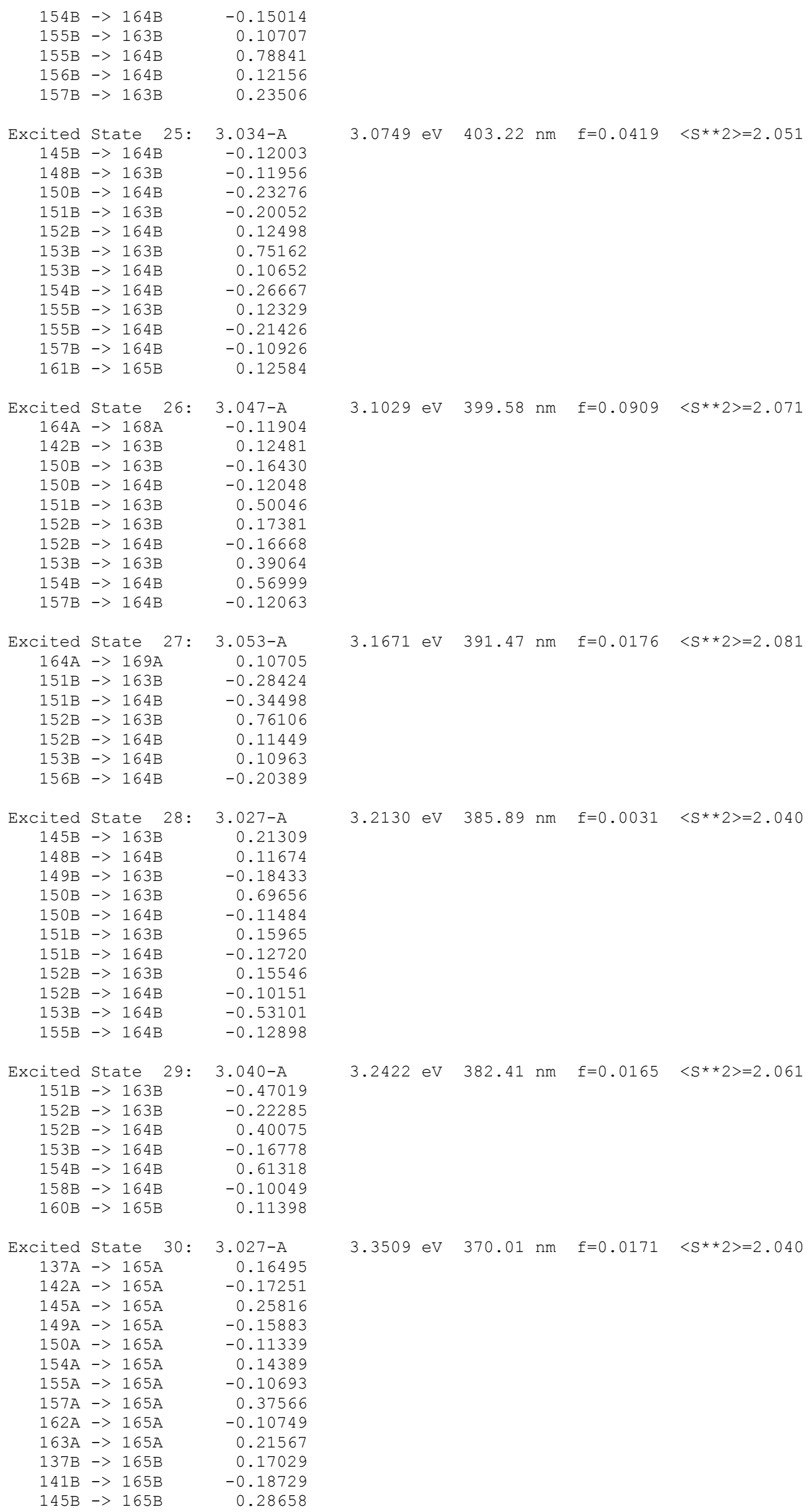

$3.0749 \mathrm{eV} \quad 403.22 \mathrm{~nm} \quad \mathrm{f}=0.0419 \quad<\mathrm{S} * * 2>=2.051$

$3.1029 \mathrm{eV} \quad 399.58 \mathrm{~nm} \quad \mathrm{f}=0.0909 \quad<* * 2>=2.071$

$3.1671 \mathrm{eV} \quad 391.47 \mathrm{~nm} \quad \mathrm{f}=0.0176 \quad\langle\mathrm{~S} * * 2\rangle=2.081$

$3.2130 \mathrm{eV} \quad 385.89 \mathrm{~nm} \quad \mathrm{f}=0.0031 \quad<* * 2>=2.040$

$3.2422 \mathrm{eV} \quad 382.41 \mathrm{~nm} \quad \mathrm{f}=0.0165 \quad<\mathrm{S} * 2>=2.061$

$3.3509 \mathrm{eV} \quad 370.01 \mathrm{~nm} \quad \mathrm{f}=0.0171 \quad<\mathrm{S} * * 2>=2.040$ 


$\begin{array}{rr}149 B \rightarrow 163 B & -0.15529 \\ 149 B \rightarrow 165 B & 0.20697 \\ 151 B \rightarrow 163 B & -0.14470 \\ 154 B \rightarrow 165 B & 0.15803 \\ 156 B->165 B & -0.16582 \\ 157 B->165 B & 0.43411\end{array}$

\section{$\mathrm{BS}^{1} \mathbf{1}^{2+}$}

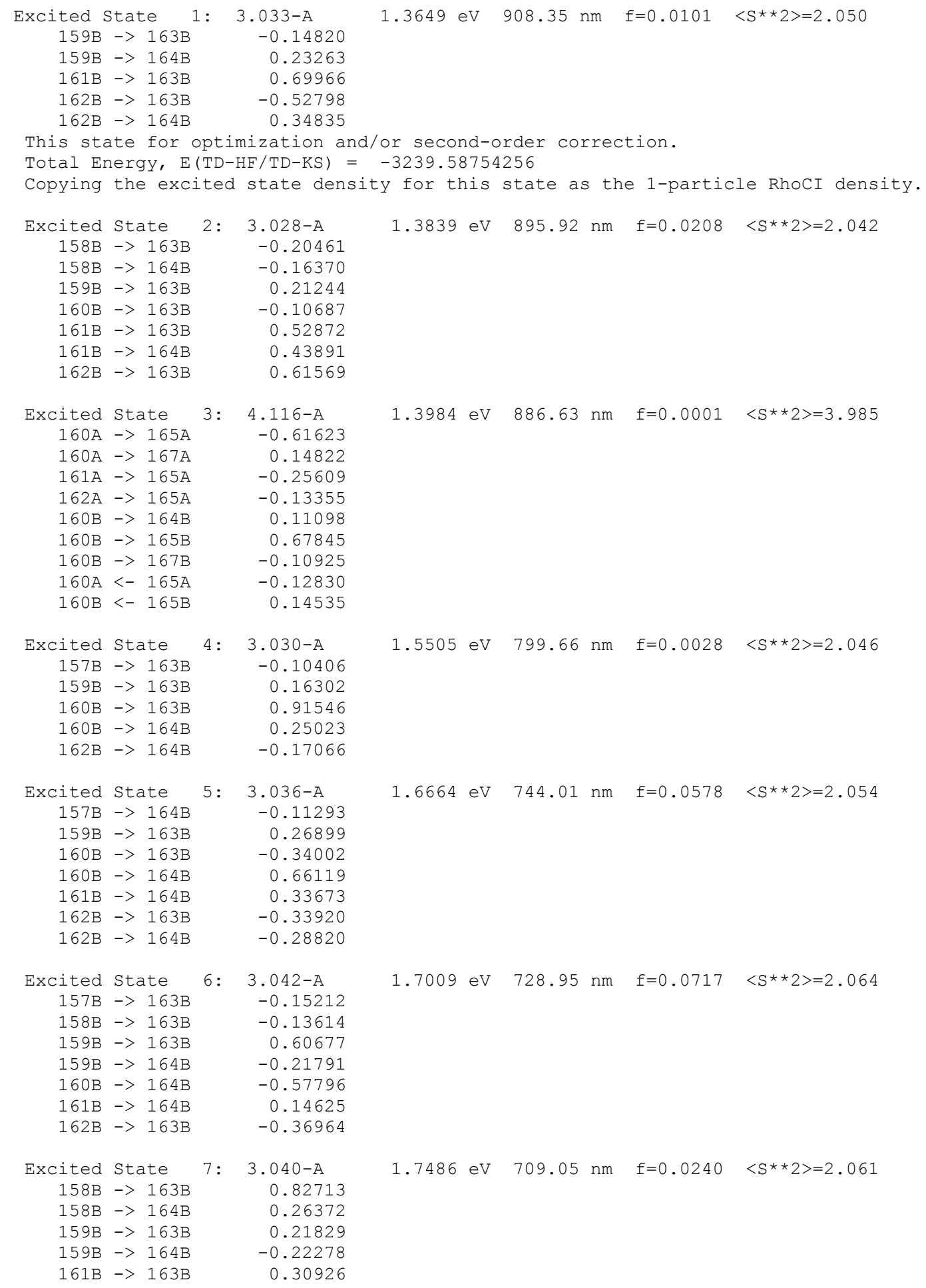




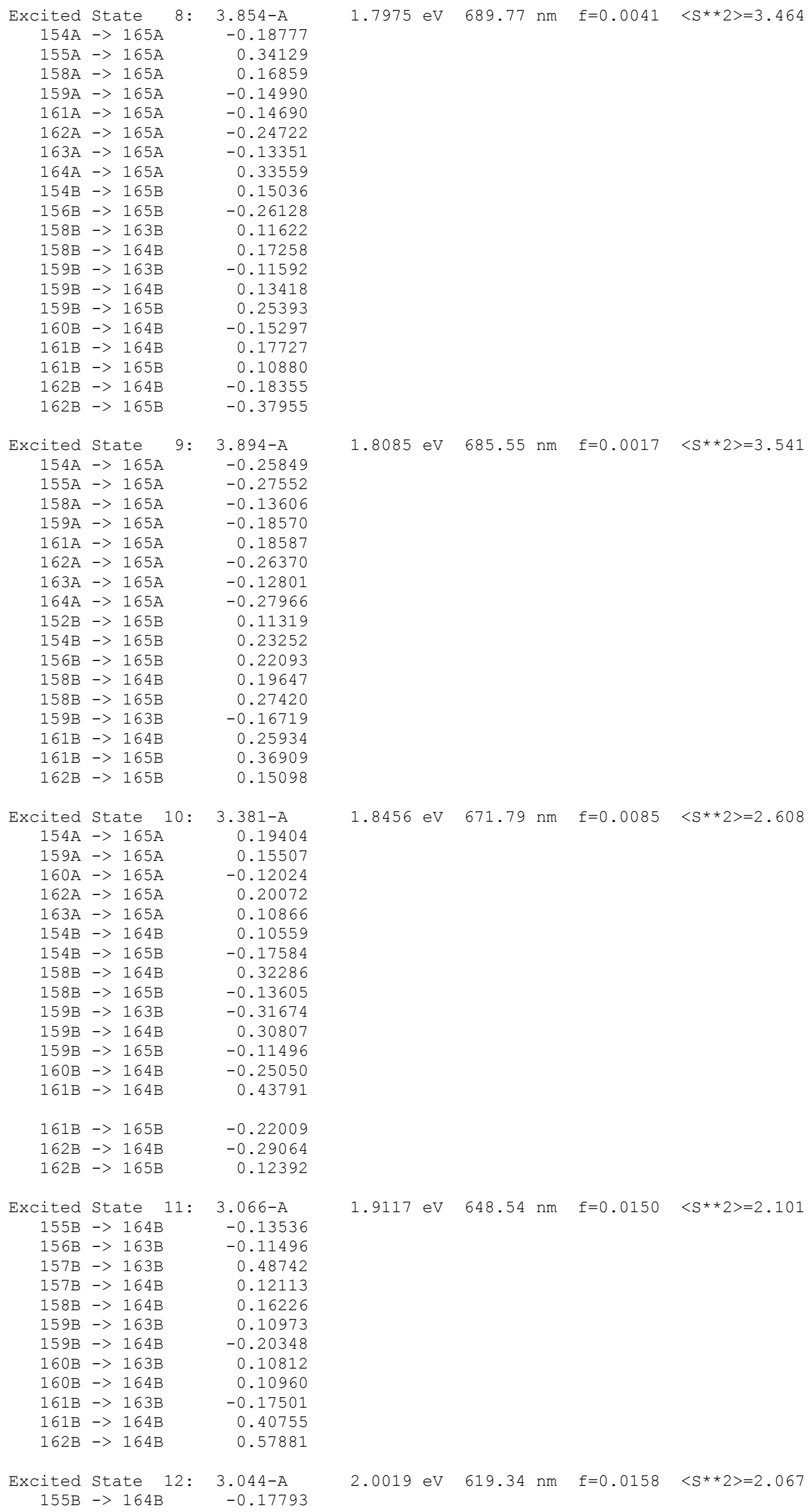




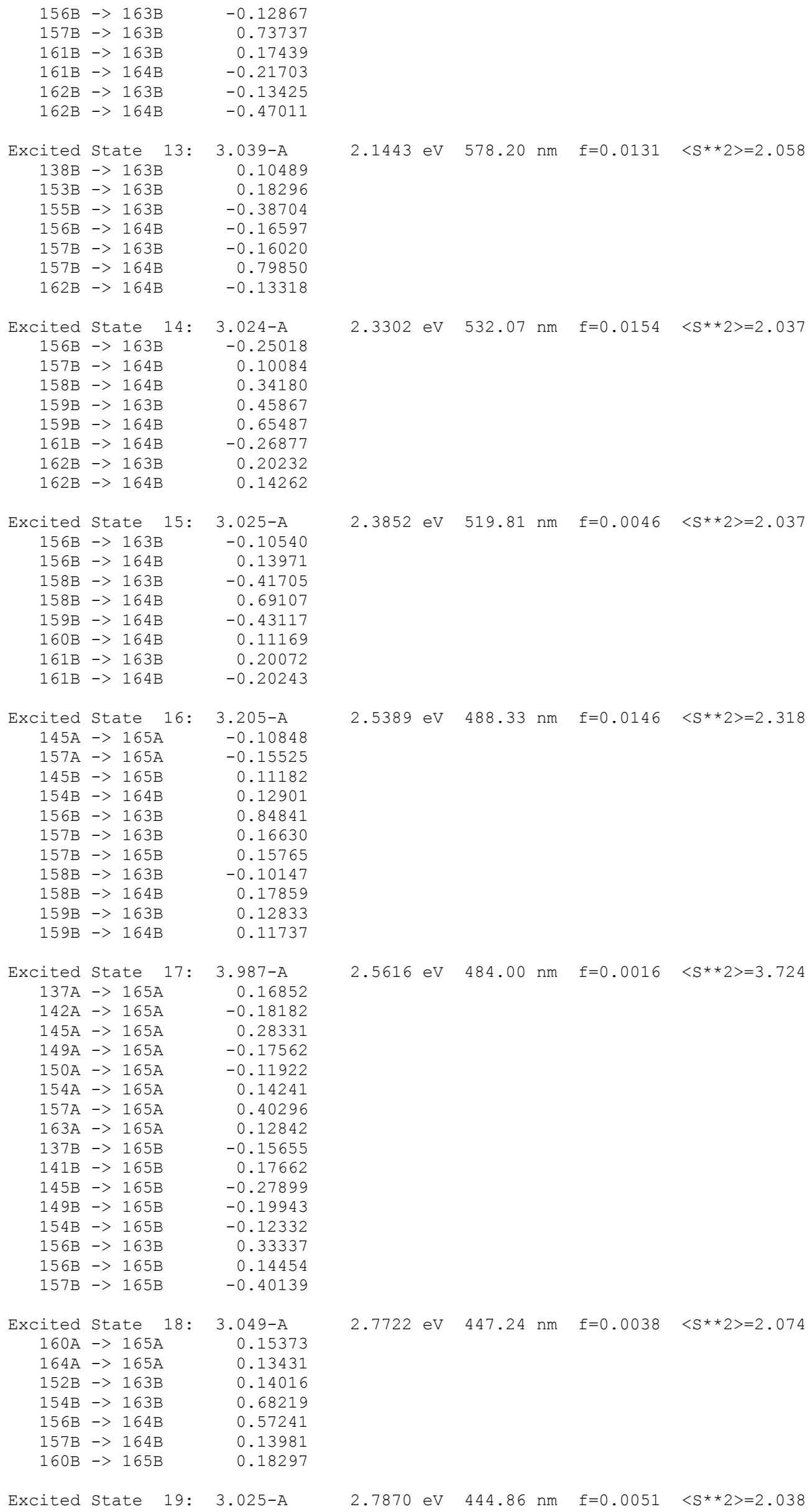




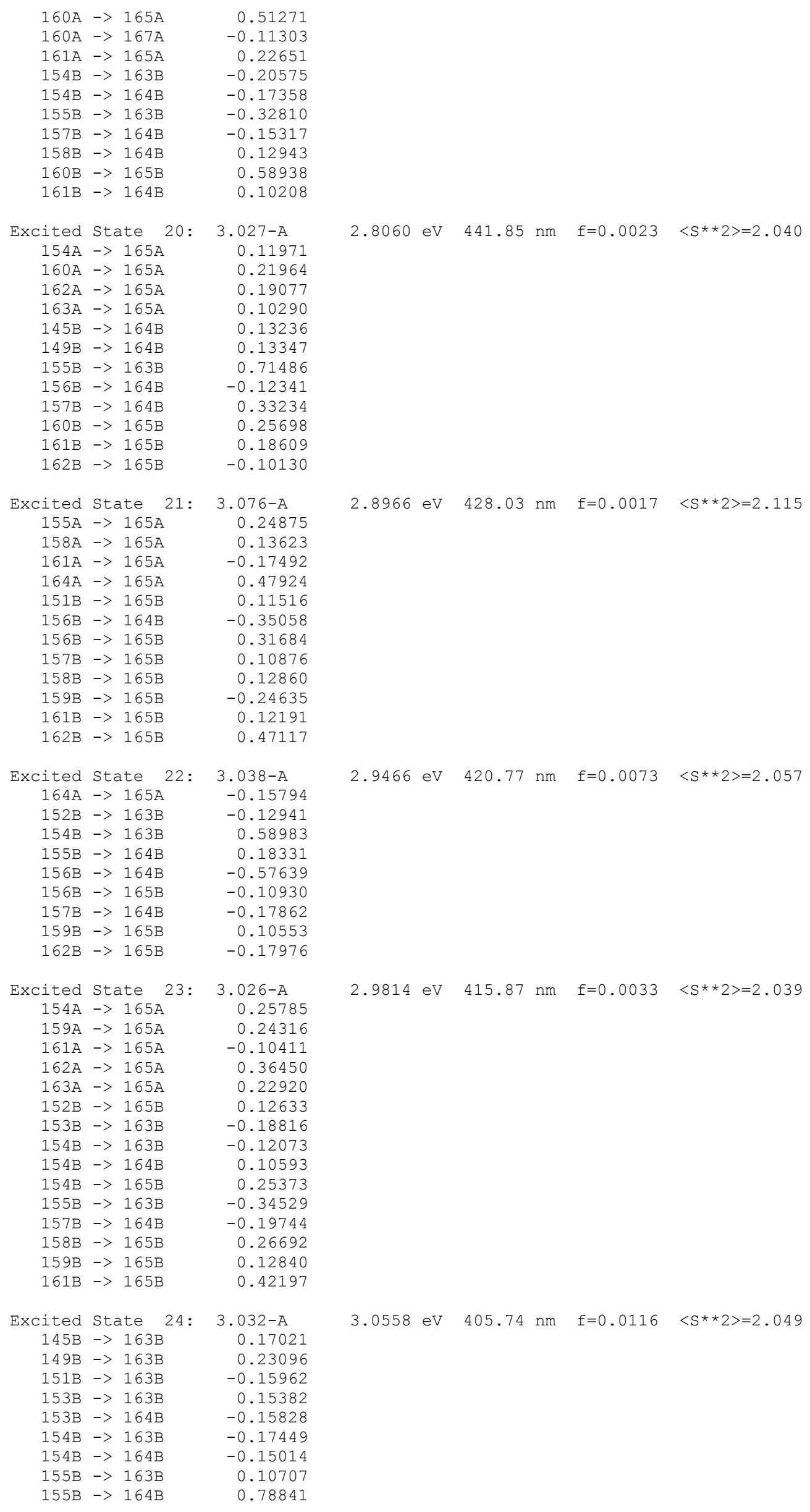




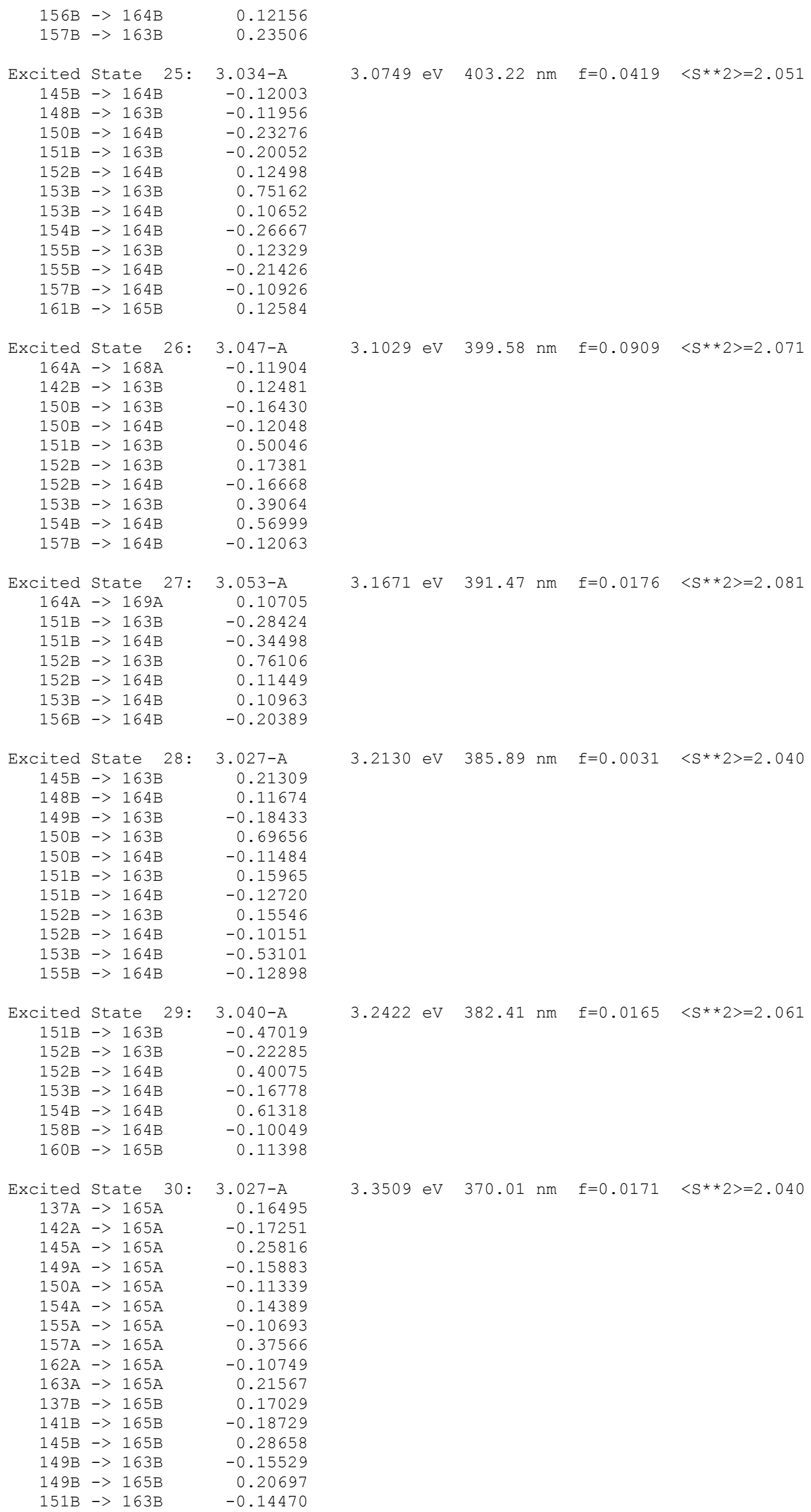




$\begin{array}{rr}154 \mathrm{~B}->165 \mathrm{~B} & 0.15803 \\ 156 \mathrm{~B}->165 \mathrm{~B} & -0.16582 \\ 157 \mathrm{~B}->165 \mathrm{~B} & 0.43411\end{array}$

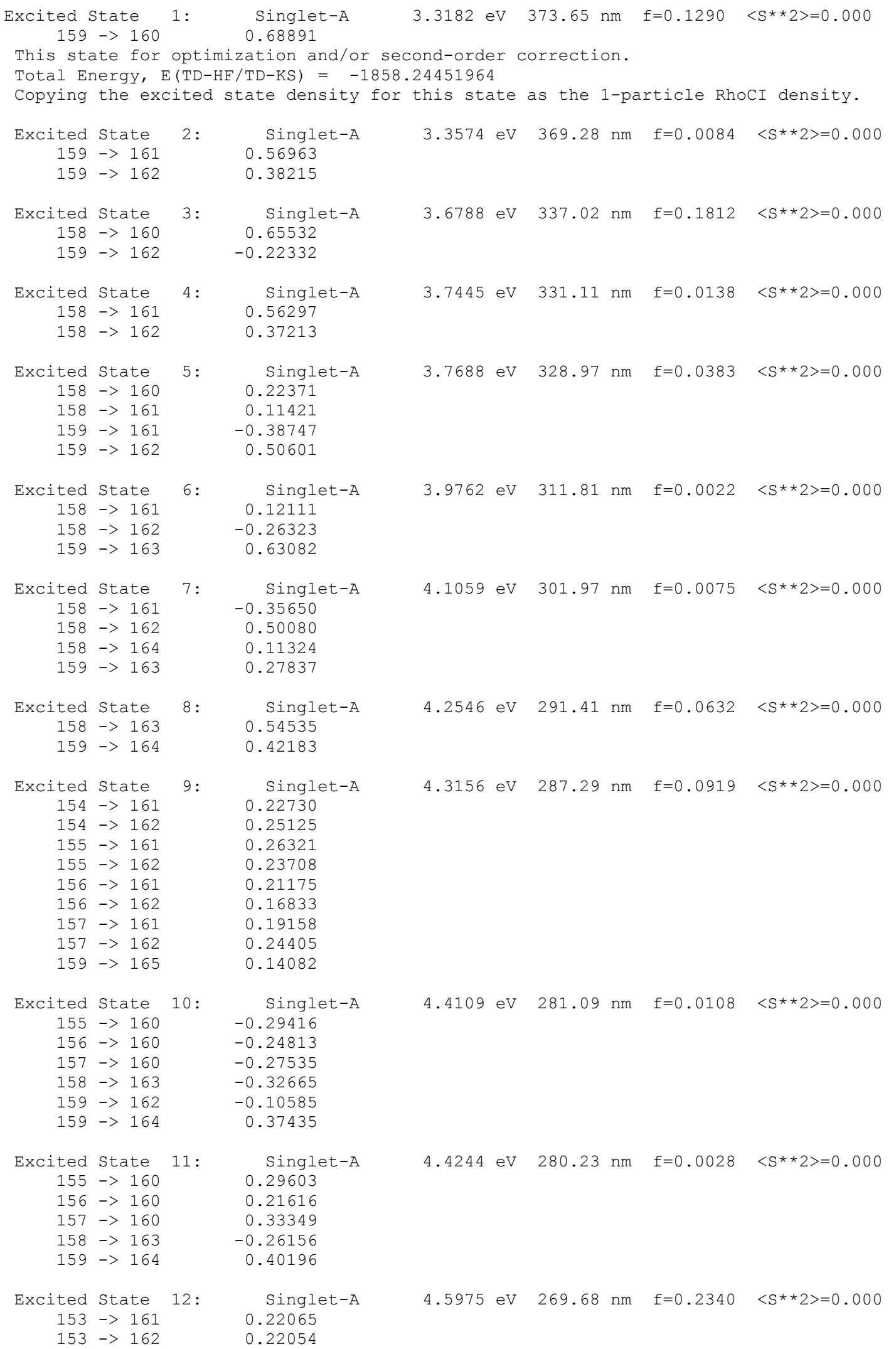




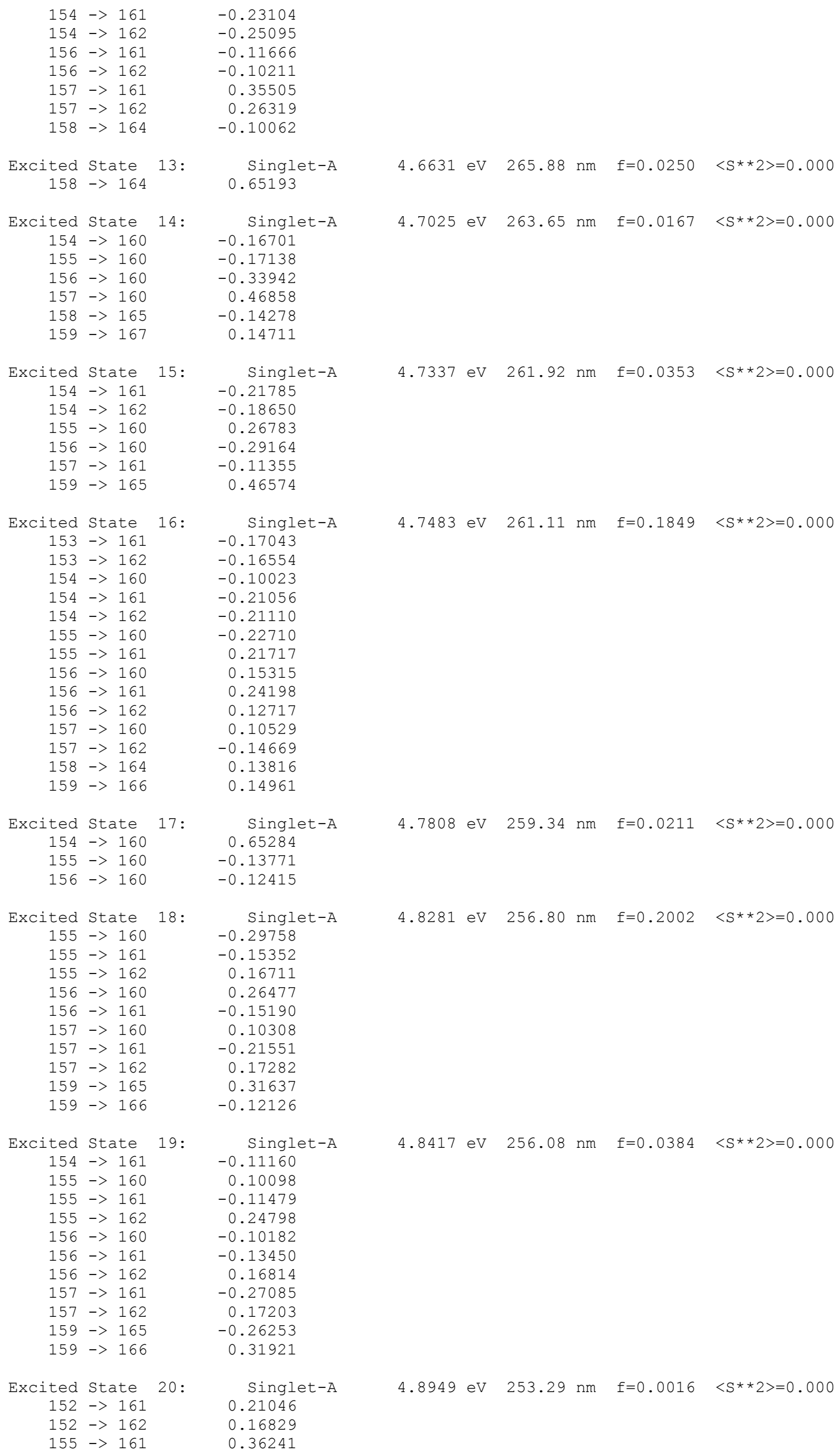




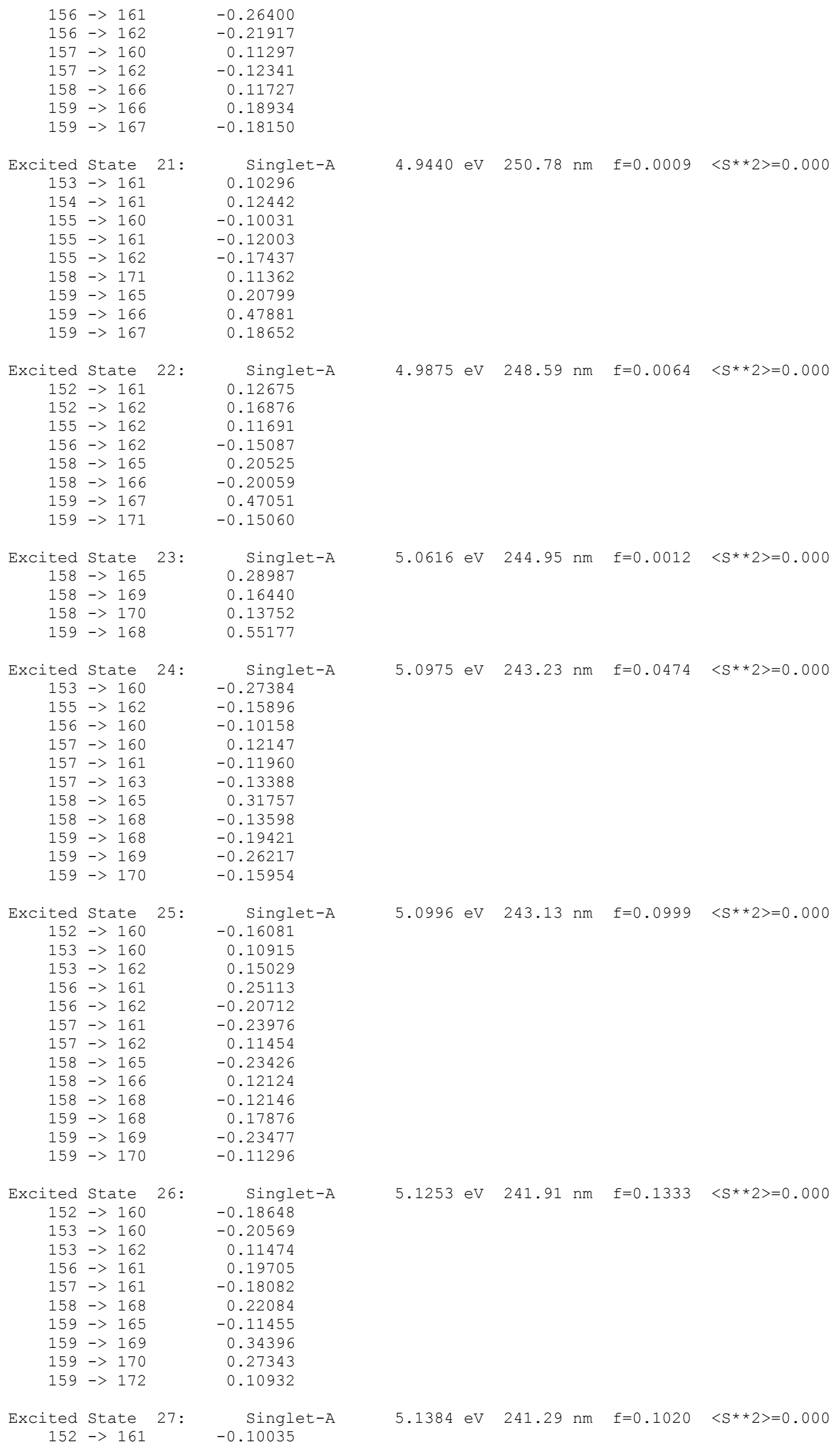




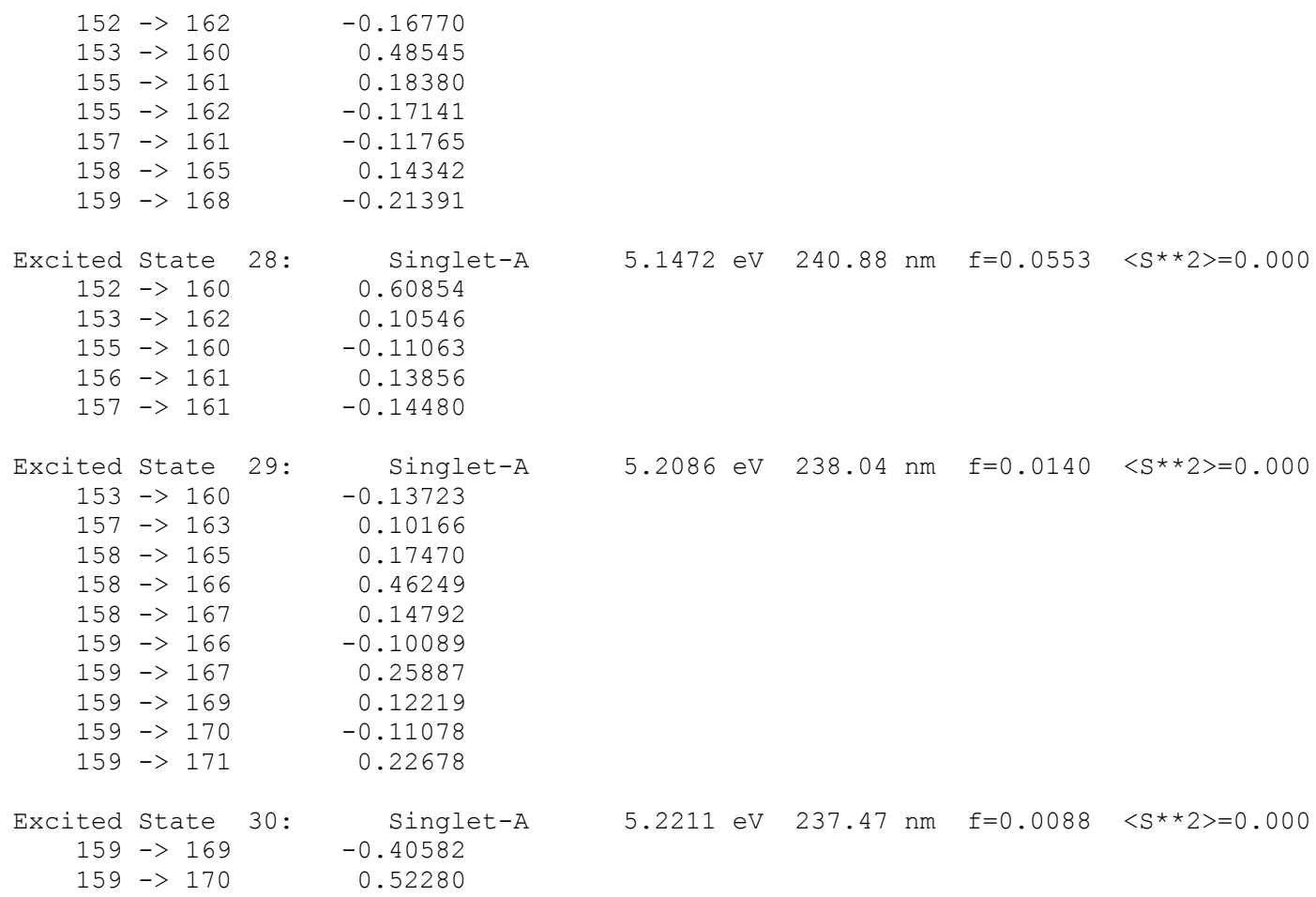




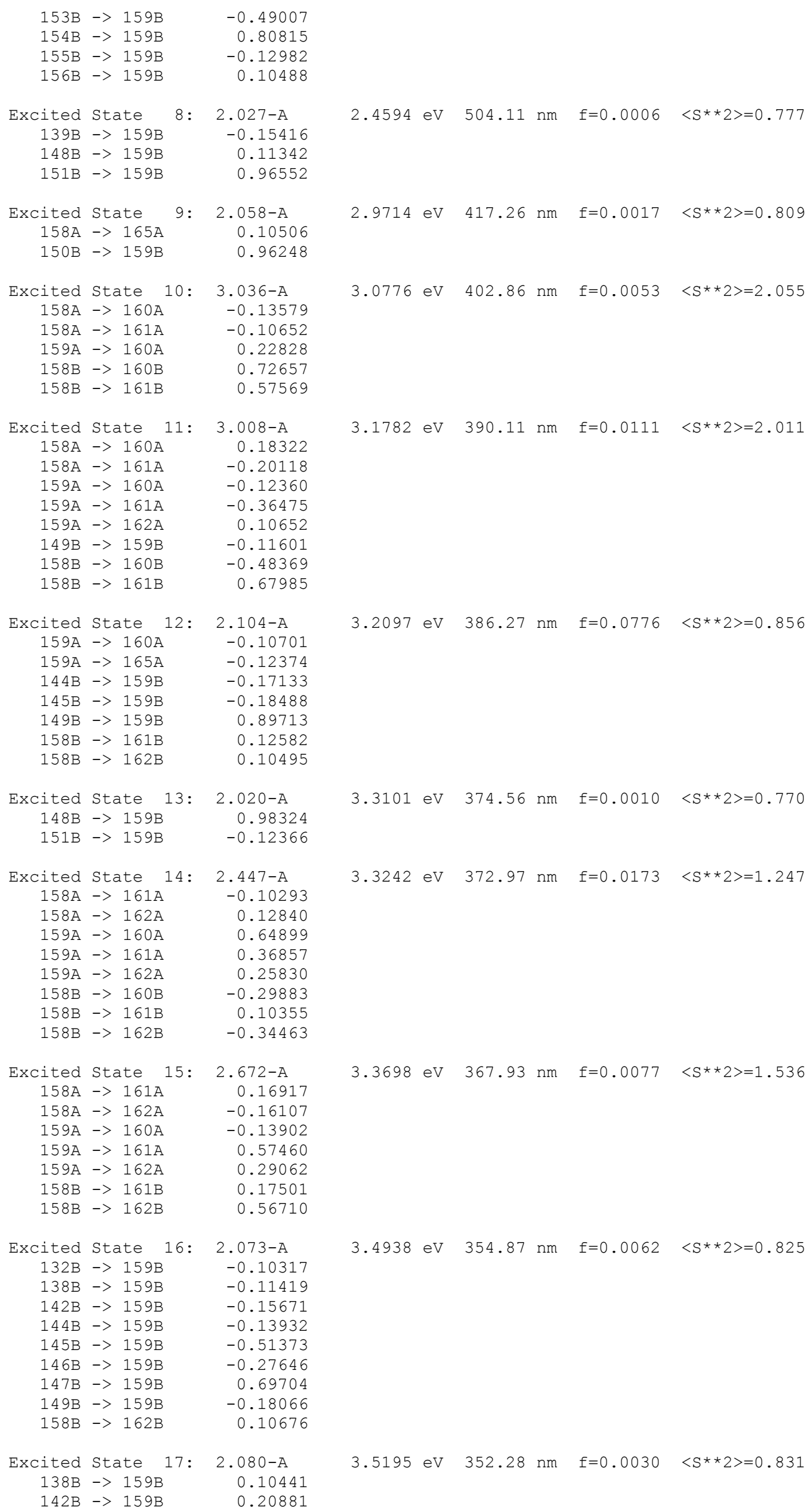

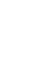

(6)




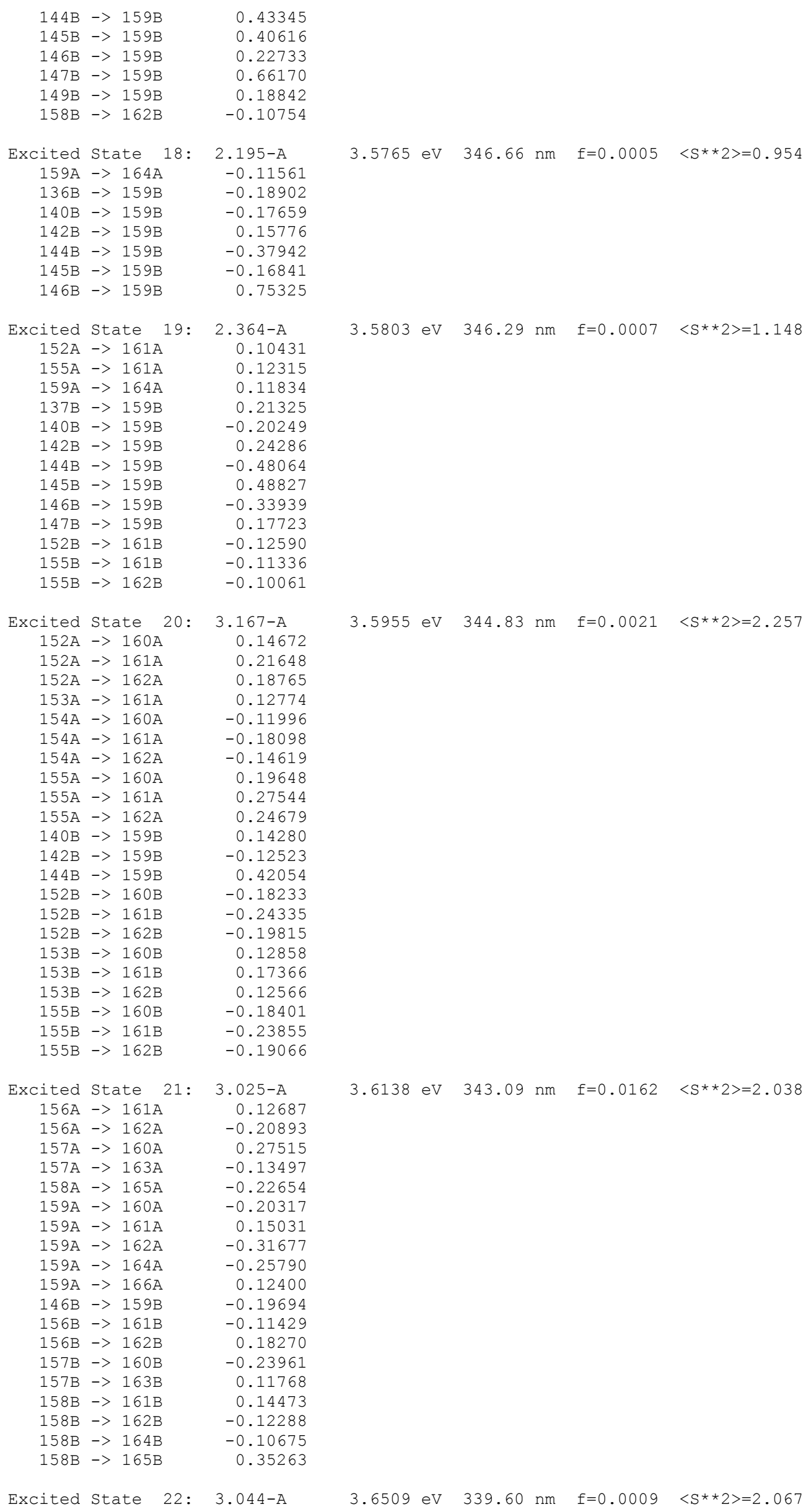




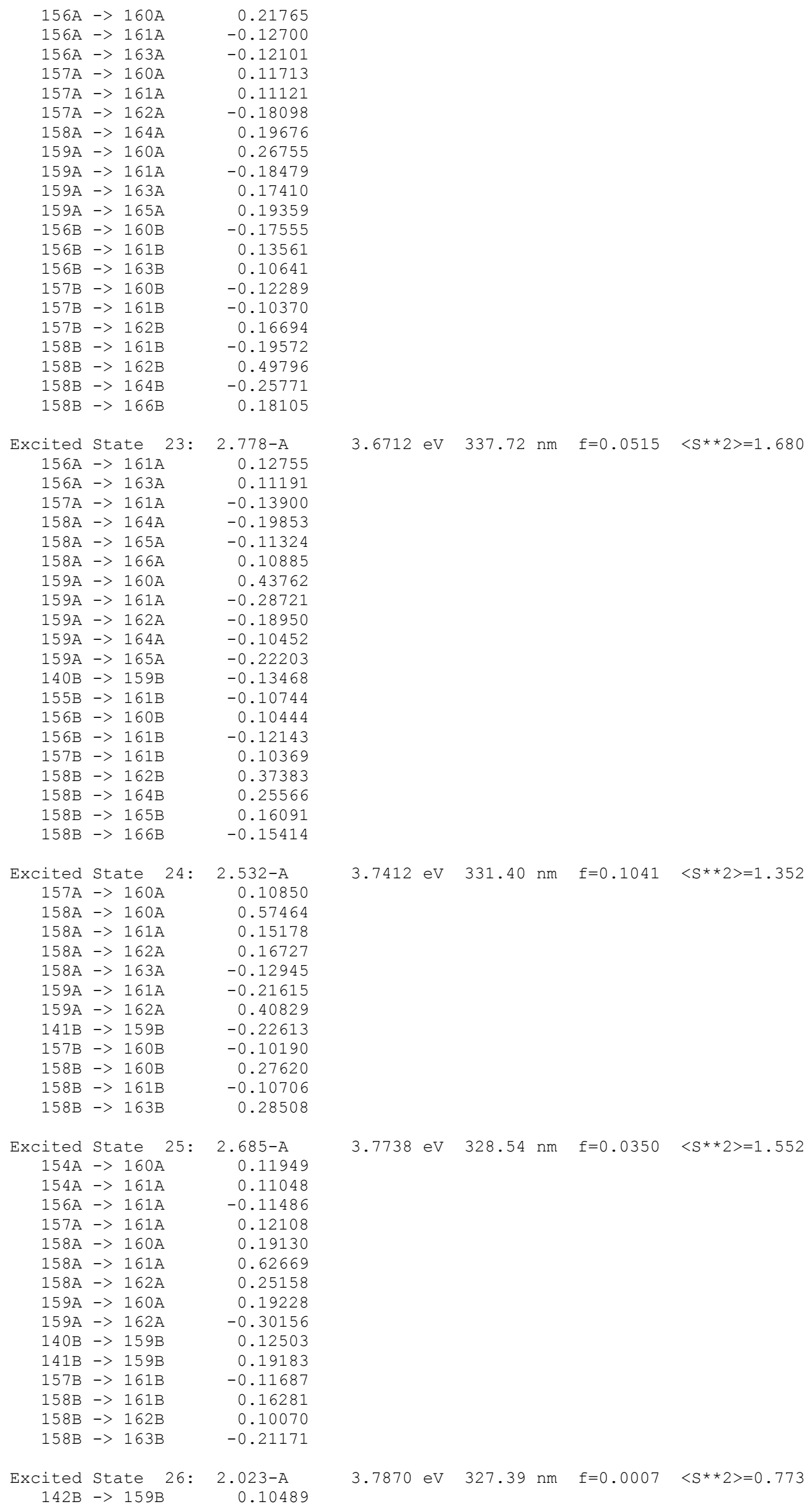




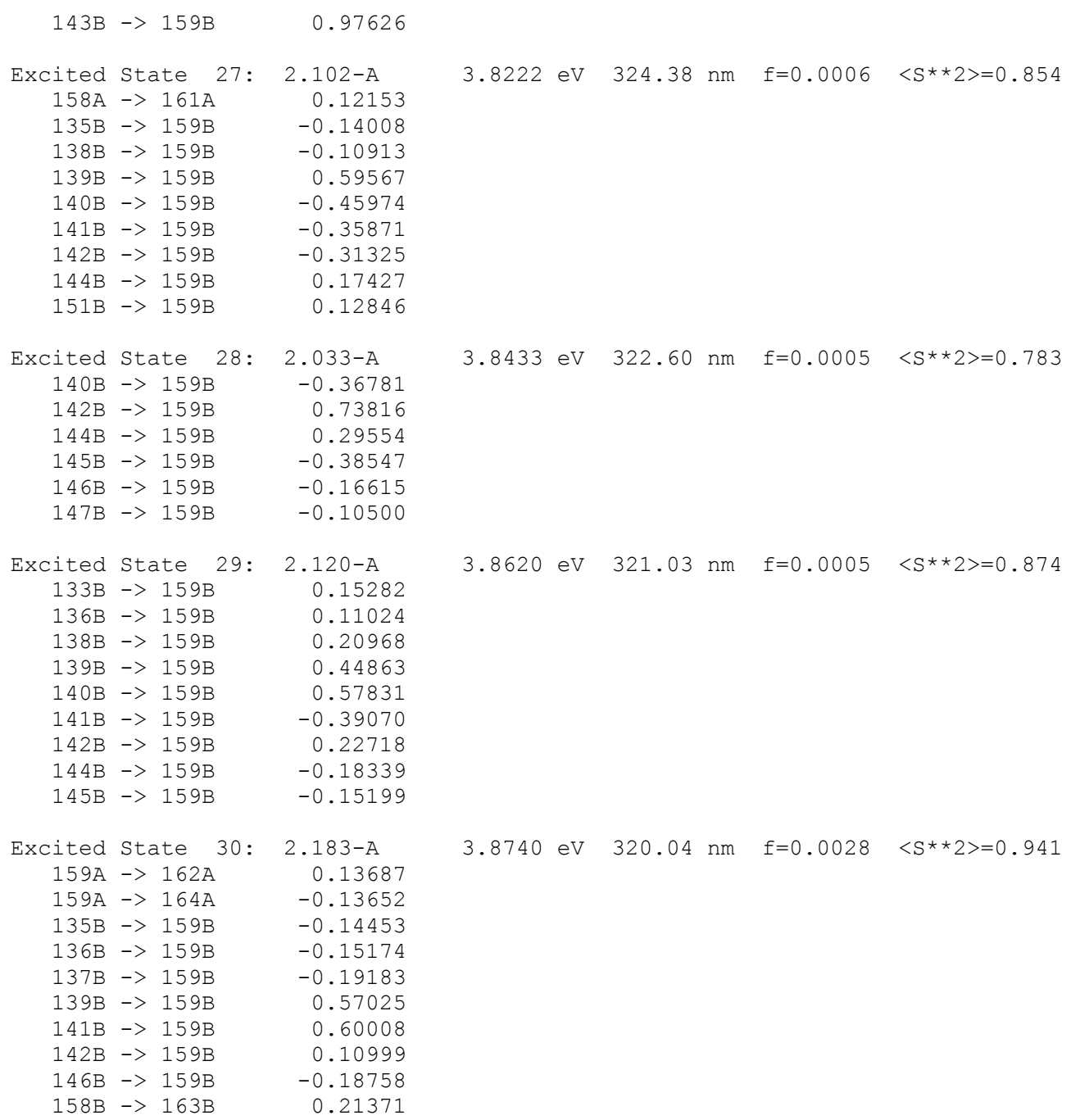




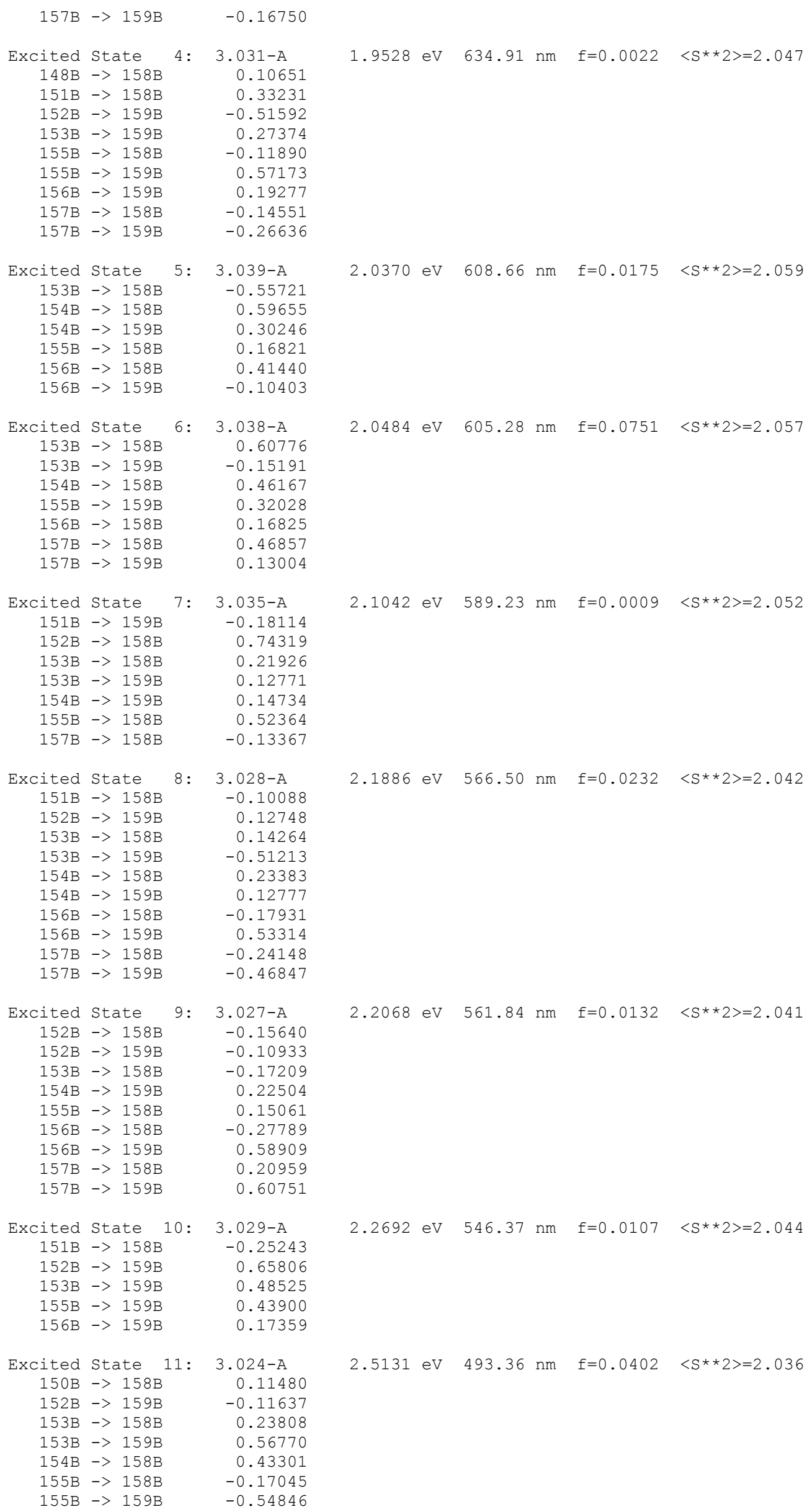




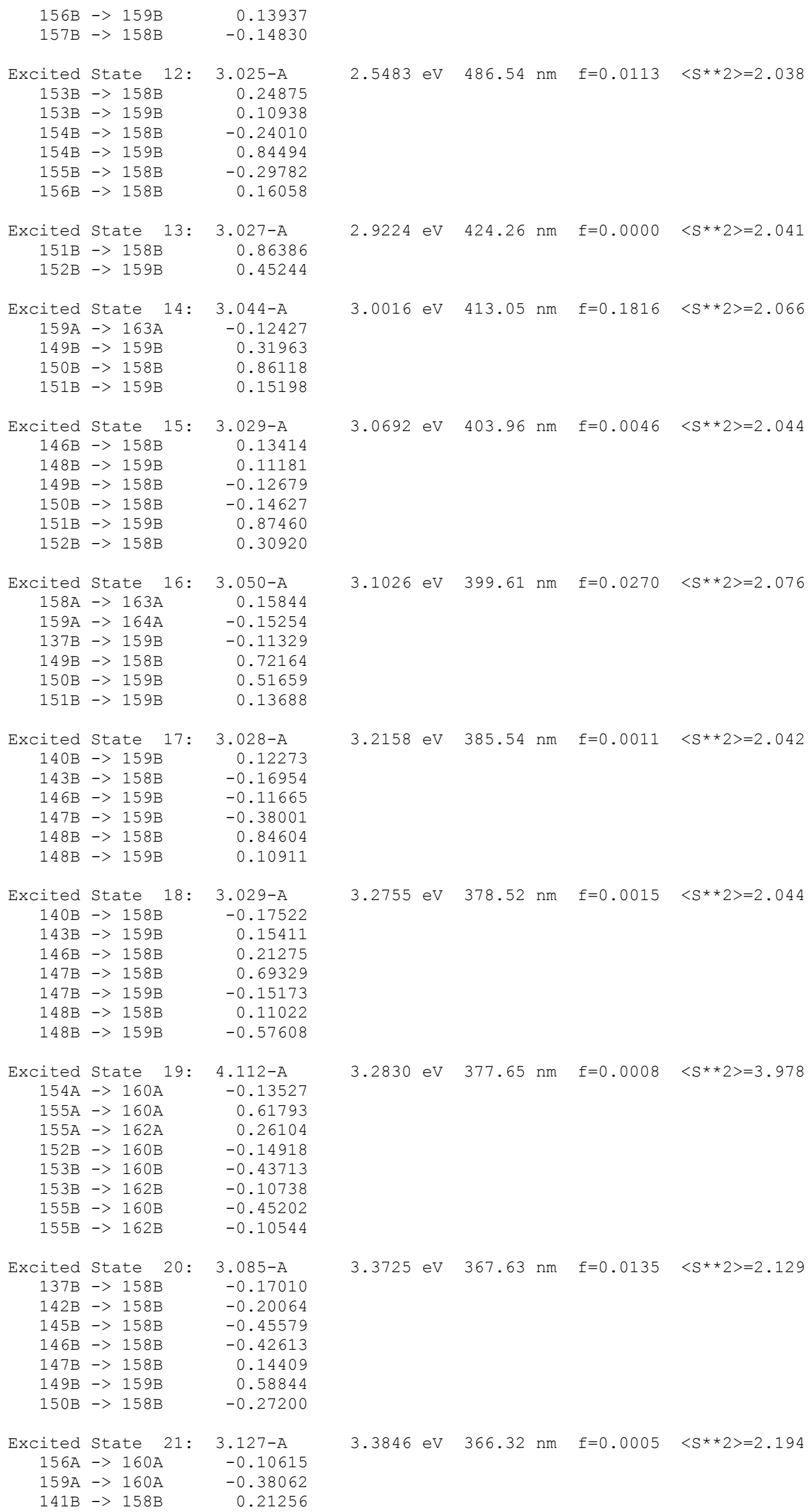




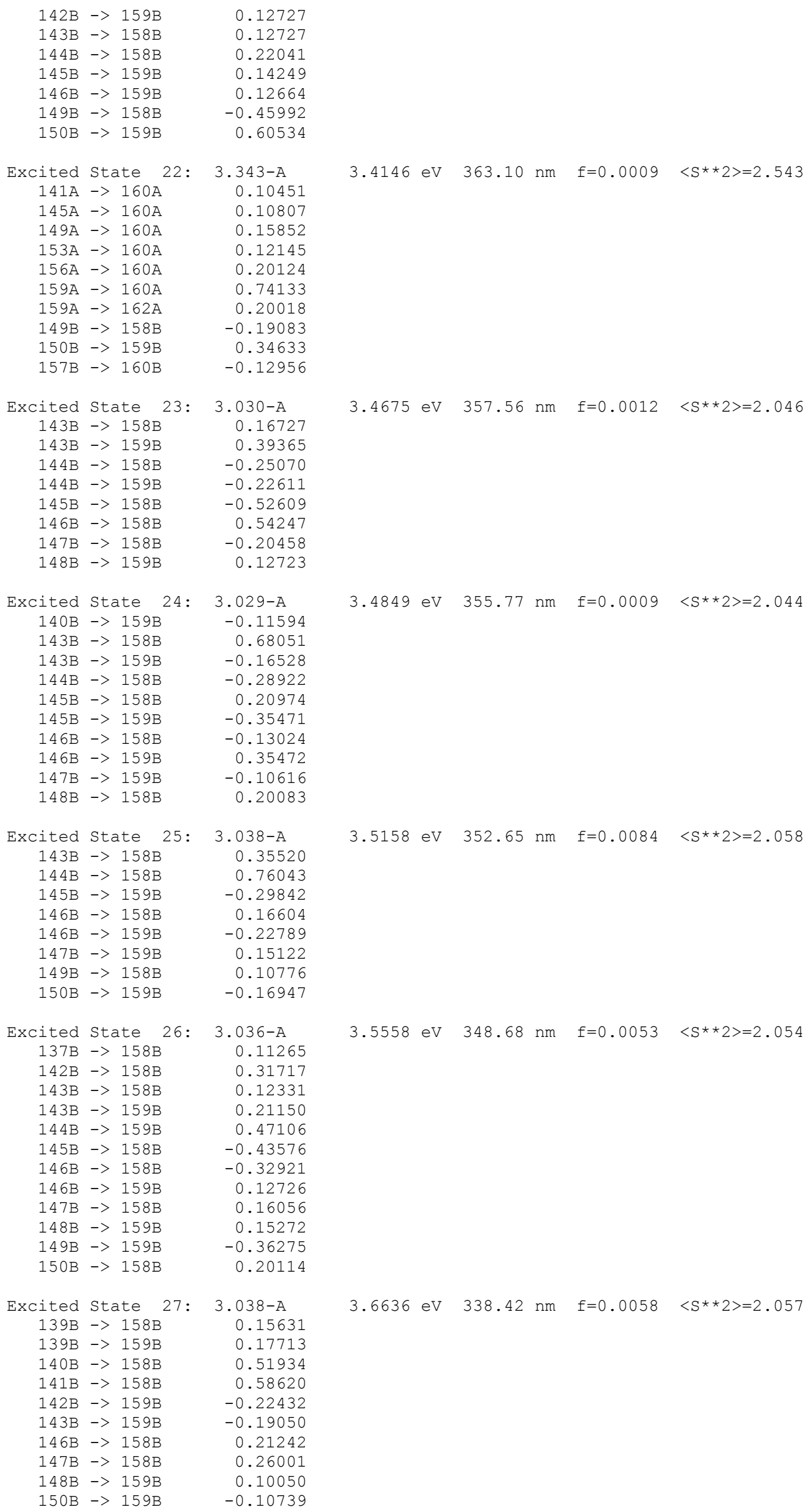




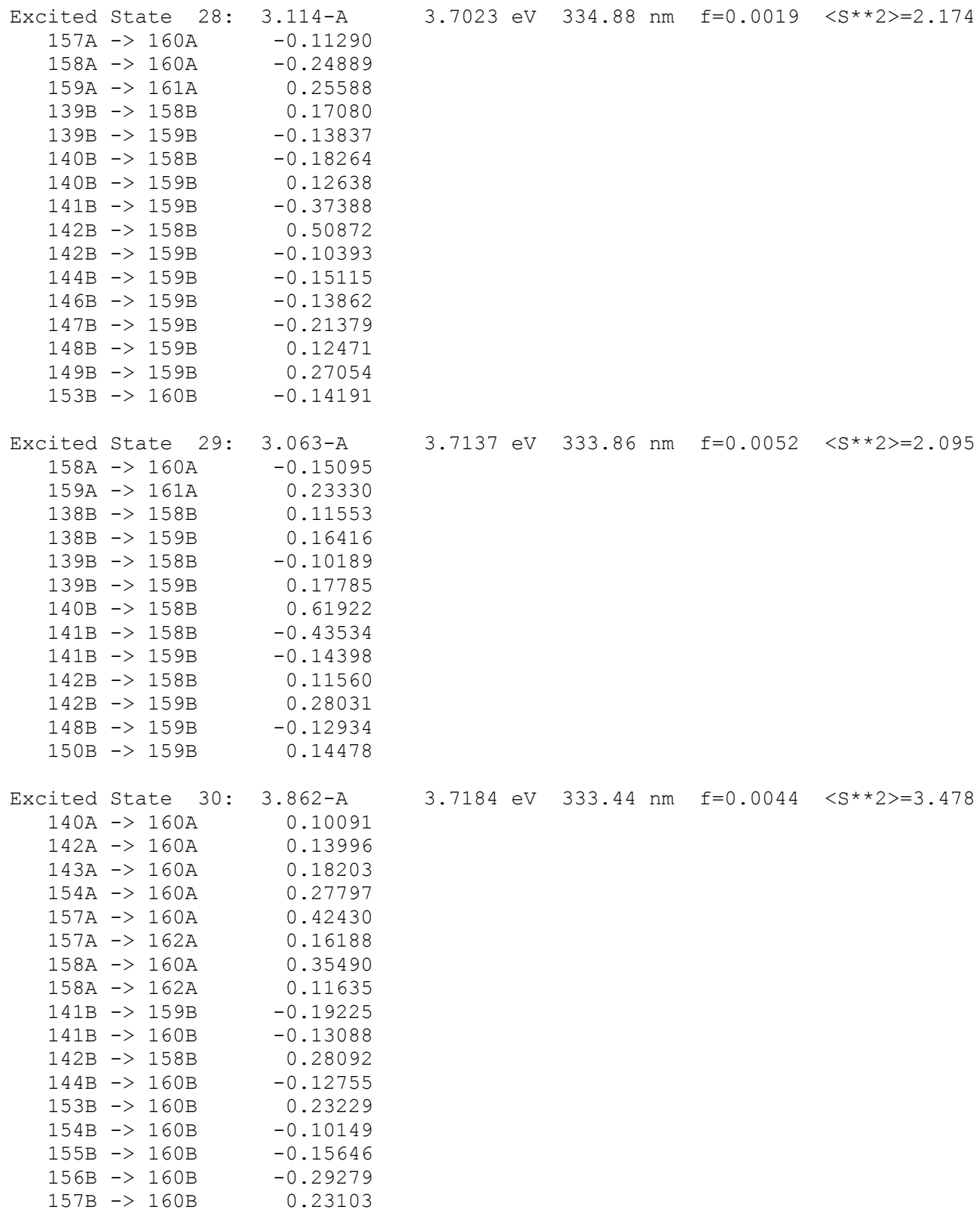

0.23103

\section{$\mathrm{BS}^{1} \mathbf{2}^{2}$}

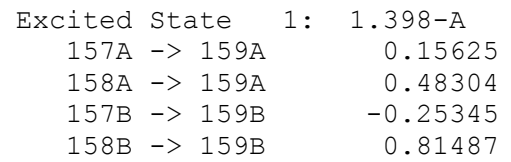

This state for optimization and/or second-order correction.

Total Energy, E(TD-HF/TD-KS) = -1857.92545843

Copying the excited state density for this state as the 1-particle RhocI density.

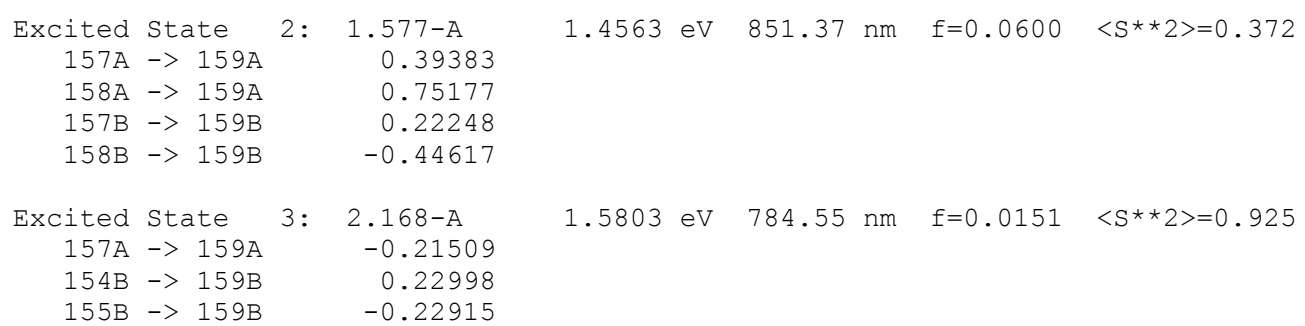




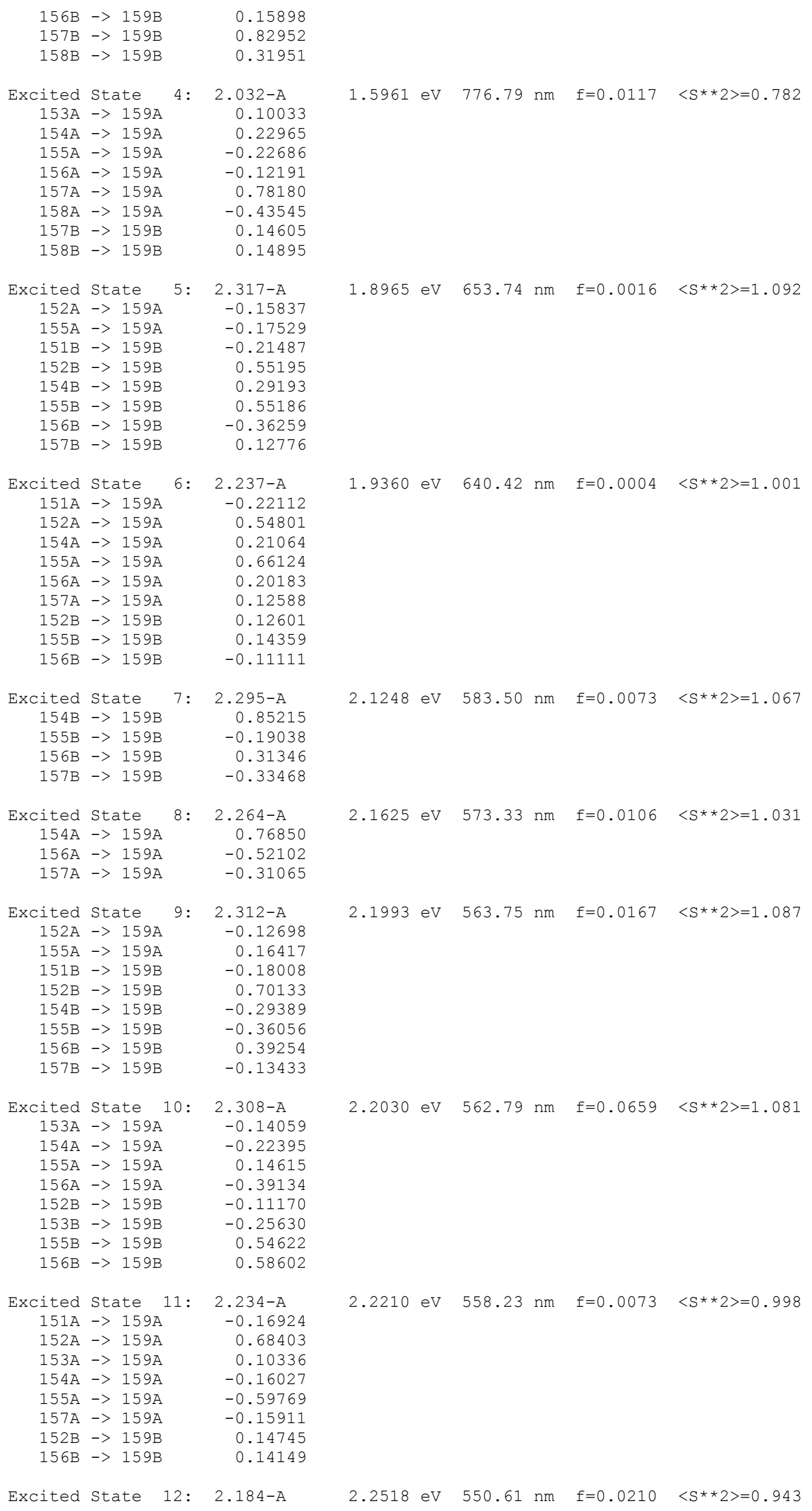

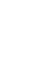

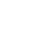




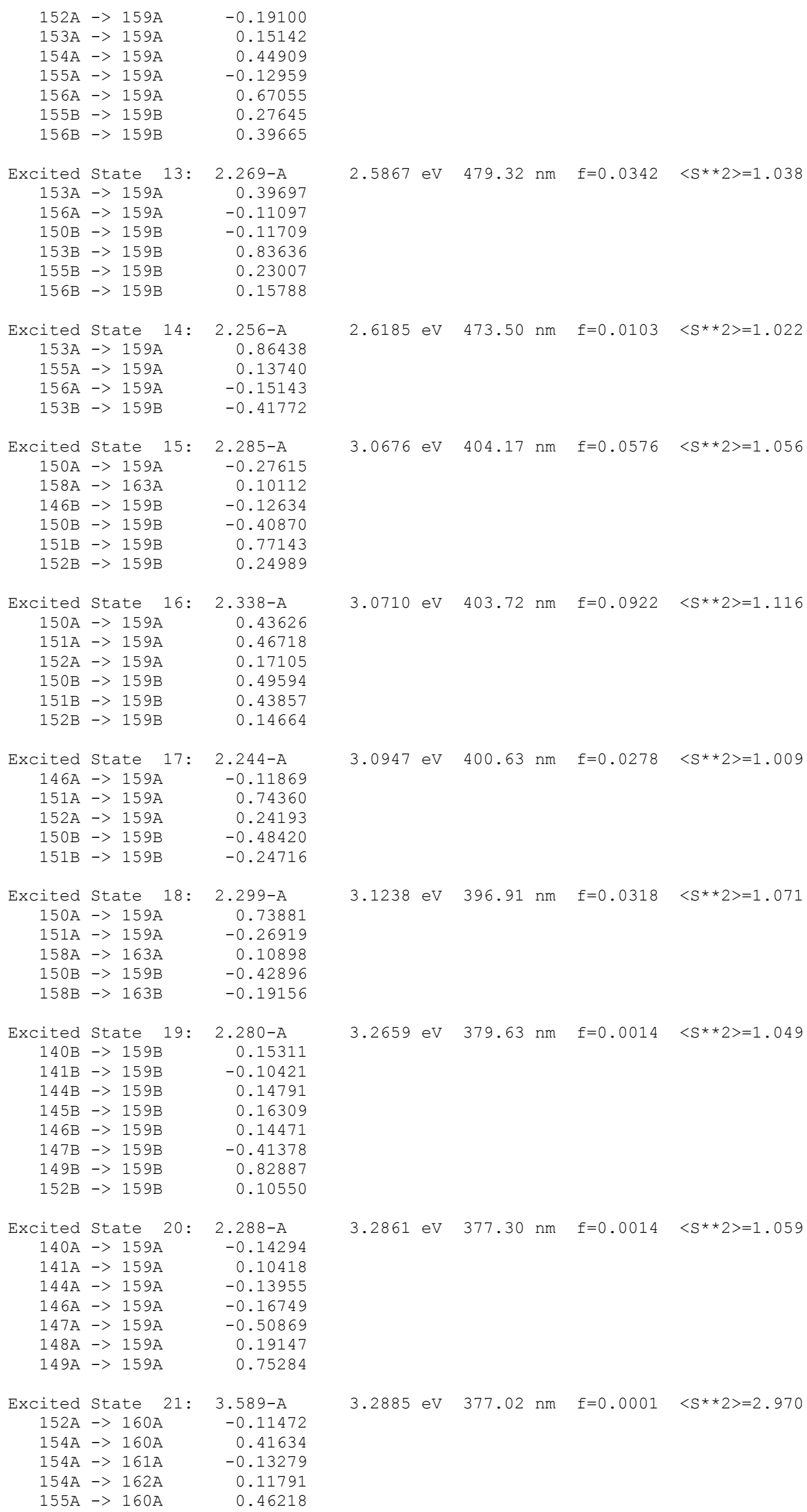




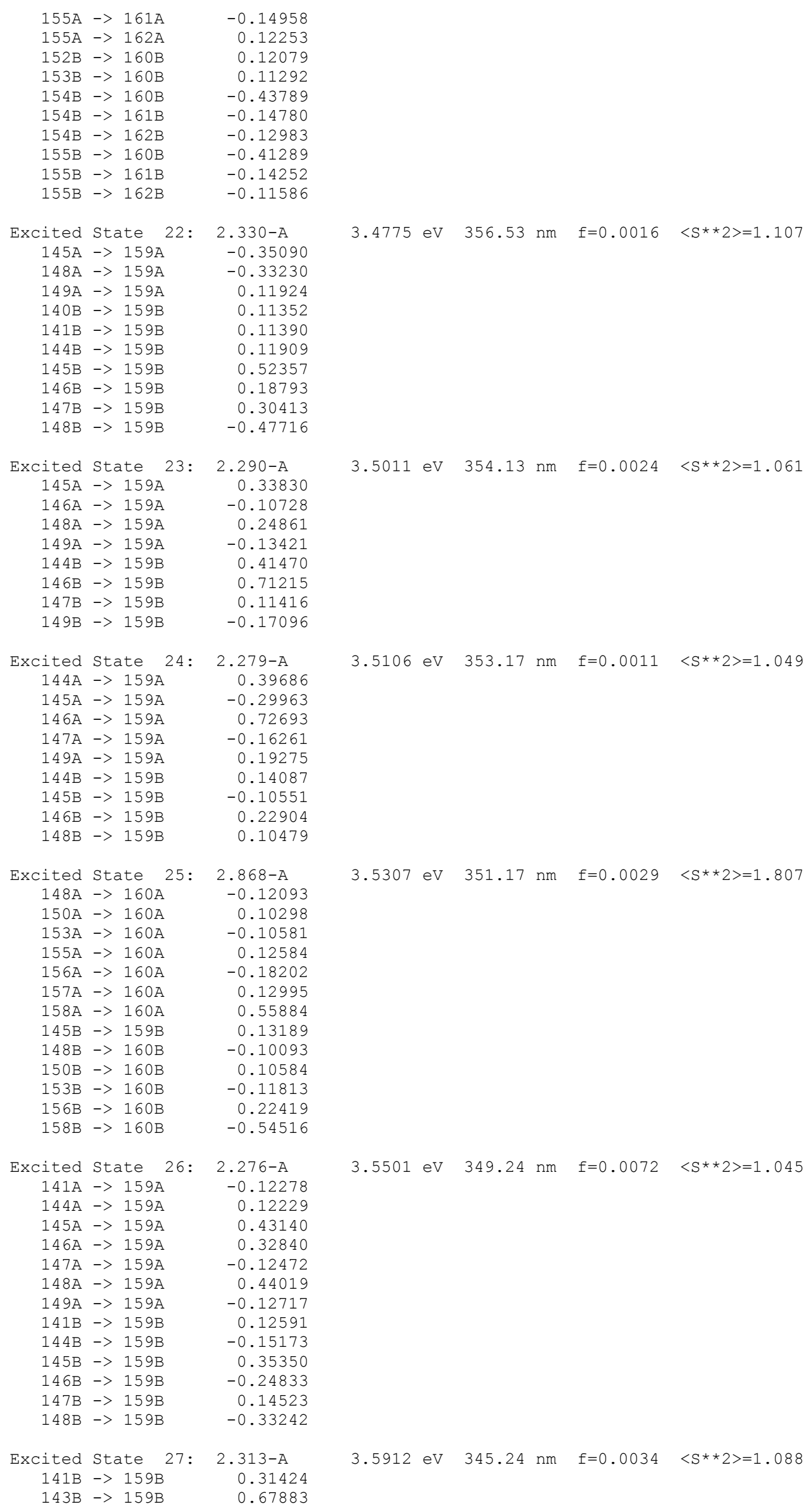




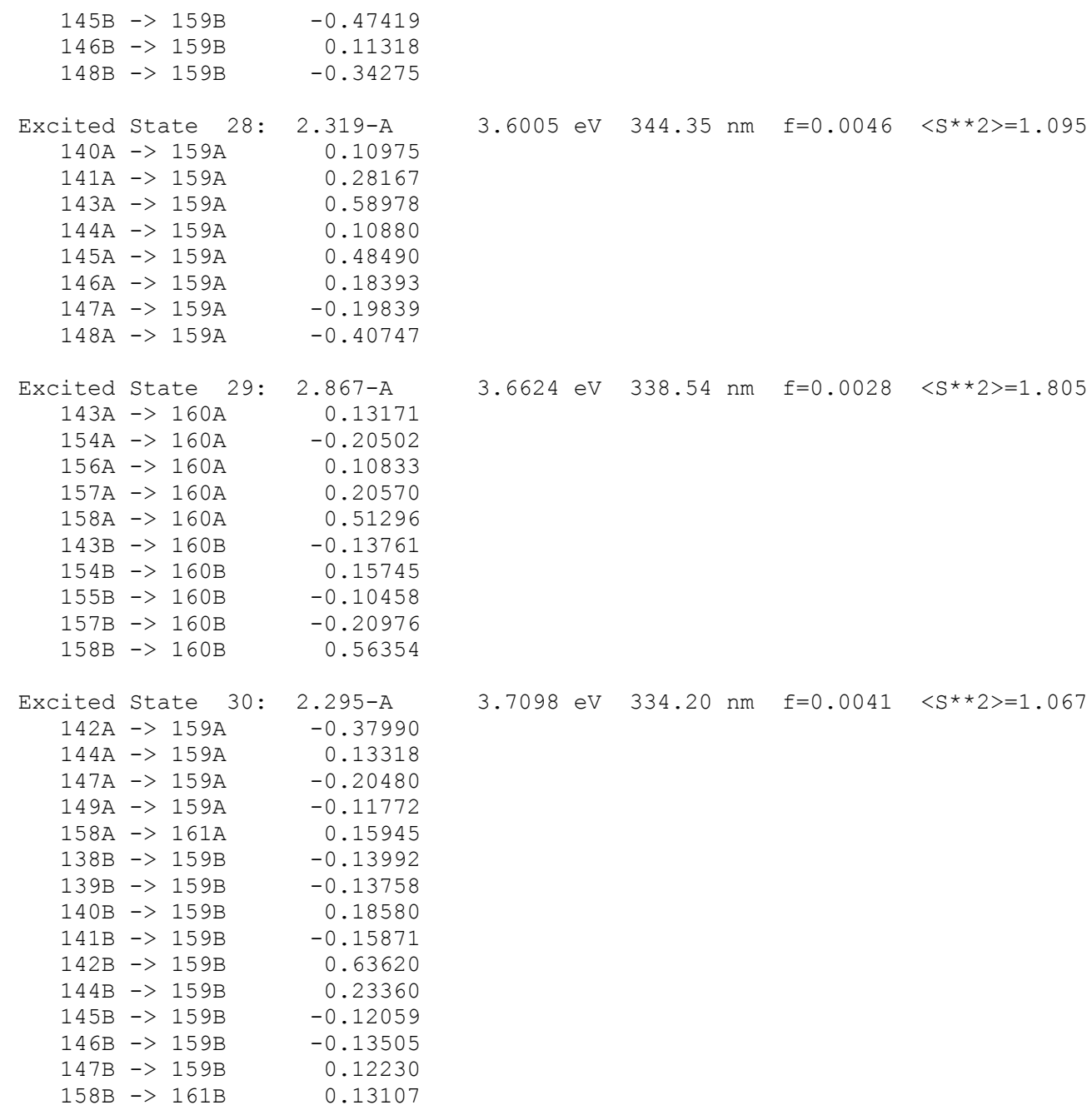

\section{3}

\begin{tabular}{|c|c|c|c|c|c|c|c|c|c|}
\hline 159 & $->161$ & & 0.69055 & & & & & & 00 \\
\hline $\begin{array}{r}\text { Excited } \\
158\end{array}$ & $\begin{array}{l}\text { State } \\
->160\end{array}$ & 3: & $\begin{array}{l}\text { Singlet-A } \\
0.69234\end{array}$ & 3.8038 & $\mathrm{eV}$ & 325.95 & $\mathrm{~nm}$ & $f=0.1233$ & $\langle S * \star 2>=0.000$ \\
\hline $\begin{array}{r}\text { Excited } \\
158 \\
159\end{array}$ & $\begin{array}{l}\text { State } \\
->161 \\
->162\end{array}$ & $4:$ & $\begin{array}{l}\text { Singlet-A } \\
-0.18190 \\
0.67464\end{array}$ & 3.9036 & $\mathrm{eV}$ & 317.61 & $\mathrm{~nm}$ & $f=0.0063$ & $<S * * 2>=0.000$ \\
\hline $\begin{array}{r}\text { Excited } \\
158 \\
159\end{array}$ & $\begin{array}{l}\text { State } \\
->161 \\
->162\end{array}$ & 5: & $\begin{aligned} & \text { Singlet-A } \\
& 0.66712 \\
& 0.17829\end{aligned}$ & 4.0529 & $\mathrm{eV}$ & 305.92 & $\mathrm{~nm}$ & $f=0.0495$ & $<S * \star 2>=0.000$ \\
\hline $\begin{array}{r}\text { Excited } \\
158 \\
159 \\
159\end{array}$ & $\begin{array}{l}\text { State } \\
->162 \\
->163 \\
->165\end{array}$ & $6:$ & $\begin{array}{rl} & \text { Singlet-A } \\
-0.22585 & 0.60637 \\
-0.21444\end{array}$ & 4.1752 & $\mathrm{eV}$ & 296.95 & $\mathrm{~nm}$ & $f=0.0948$ & $<S * * 2>=0.000$ \\
\hline $\begin{array}{r}\text { Excited } \\
155 \\
157 \\
158\end{array}$ & $\begin{array}{l}\text { State } \\
->160 \\
->160 \\
->162\end{array}$ & $7:$ & $\begin{array}{l}\text { Singlet-A } \\
-0.15616 \\
0.17106 \\
0.46104\end{array}$ & 4.3501 & $\mathrm{eV}$ & 285.01 & $\mathrm{~nm}$ & $f=0.0065$ & $<S * * 2>=0.000$ \\
\hline
\end{tabular}




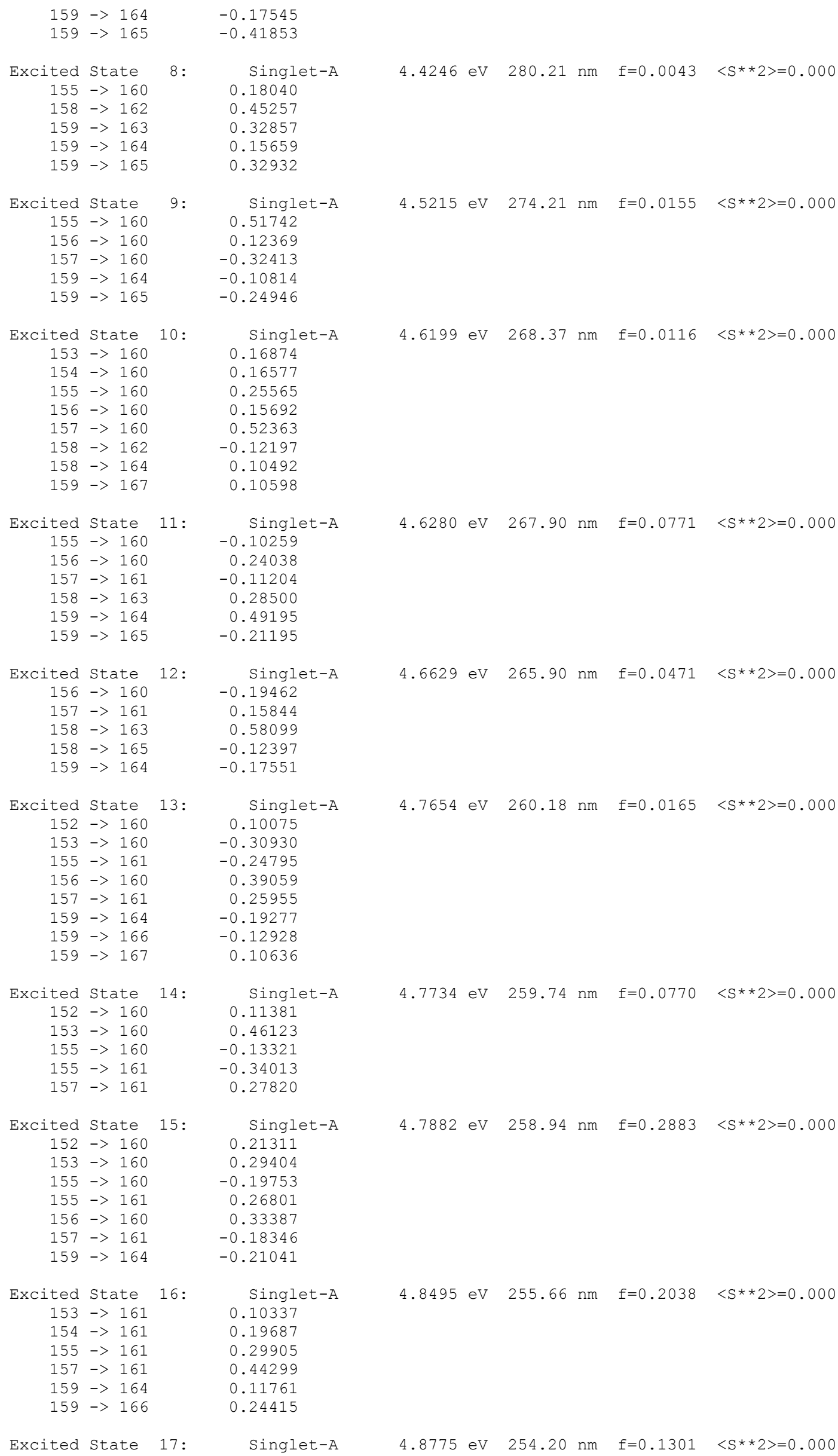




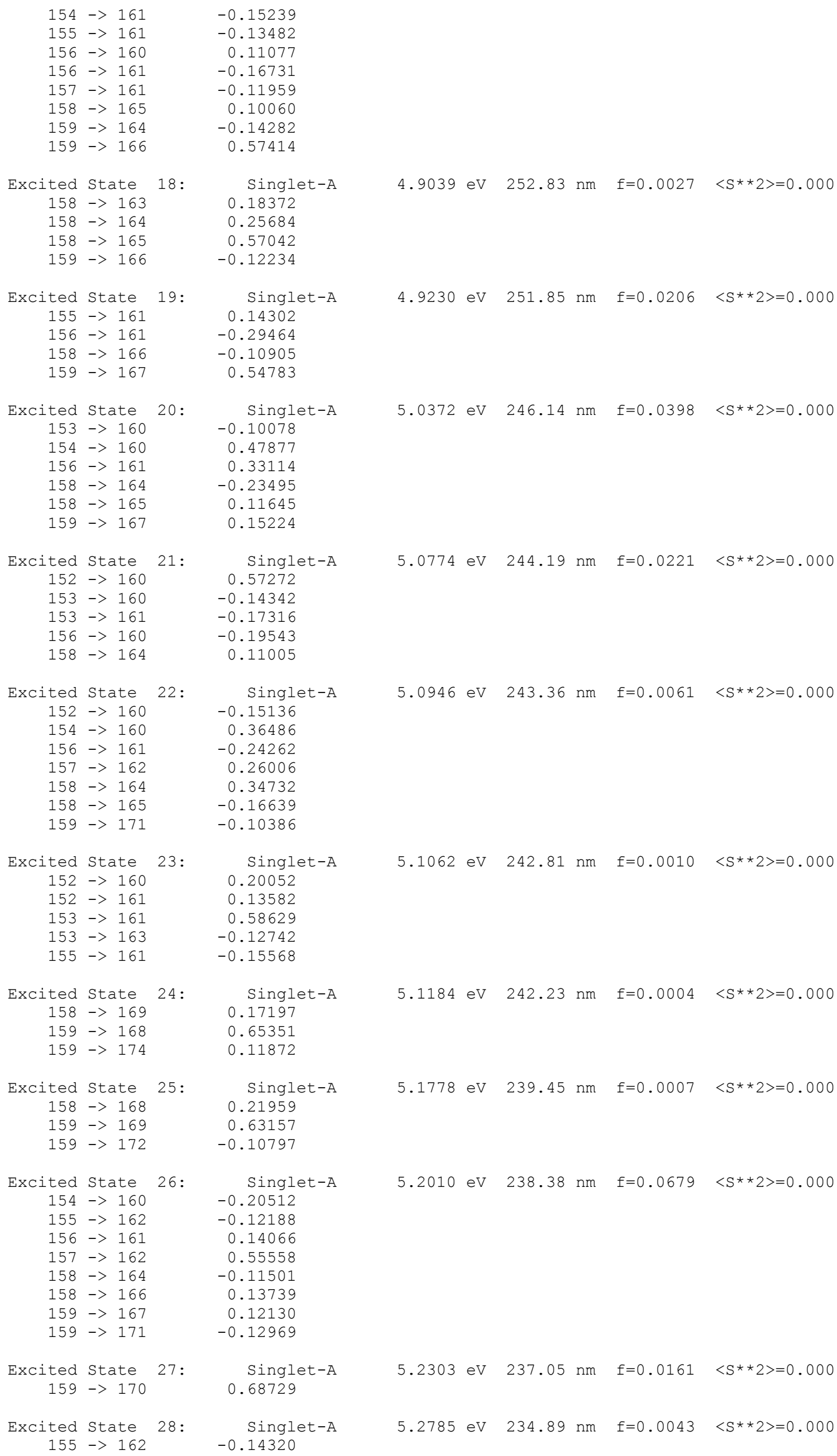




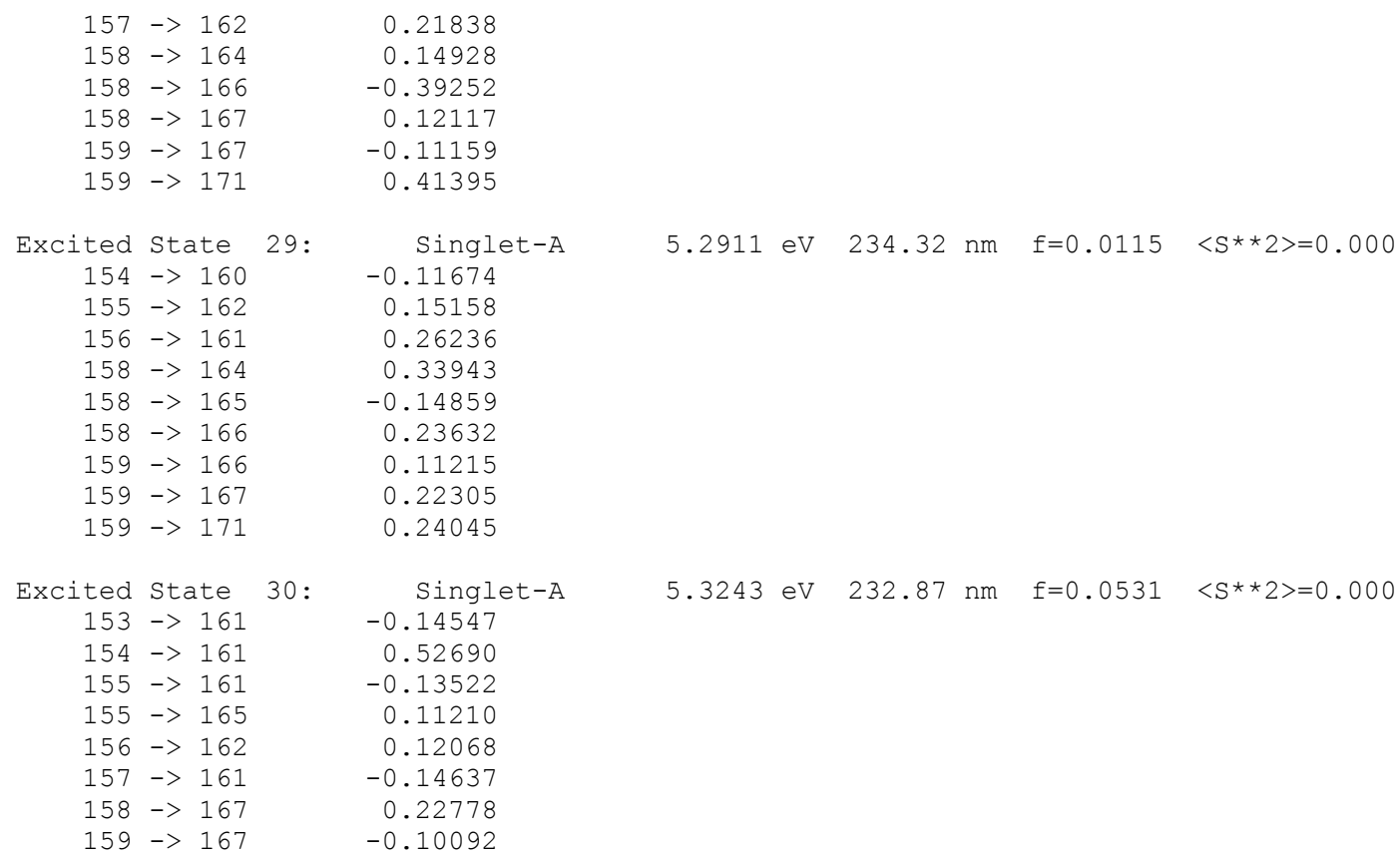

\section{$3^{+}$}

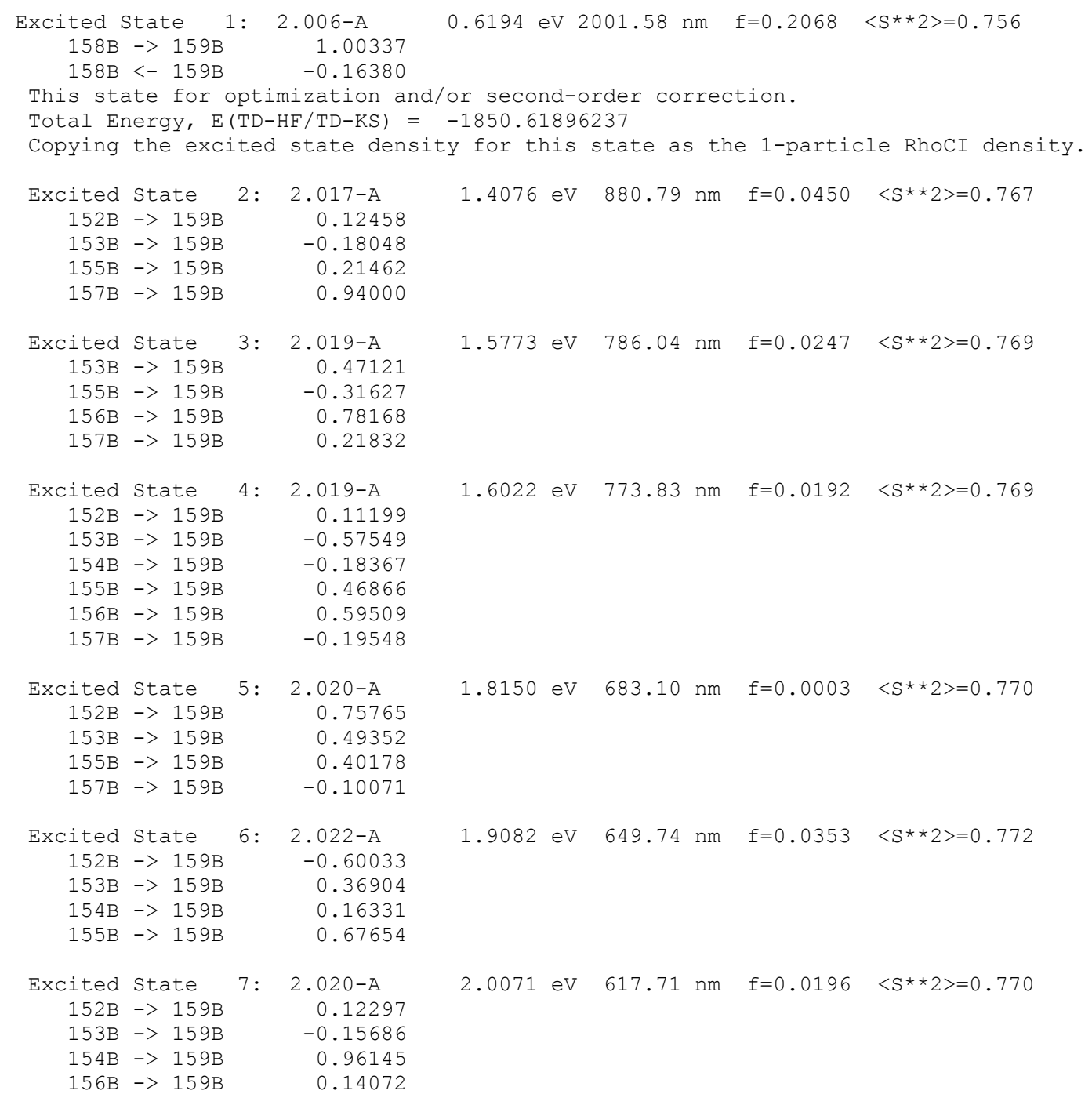




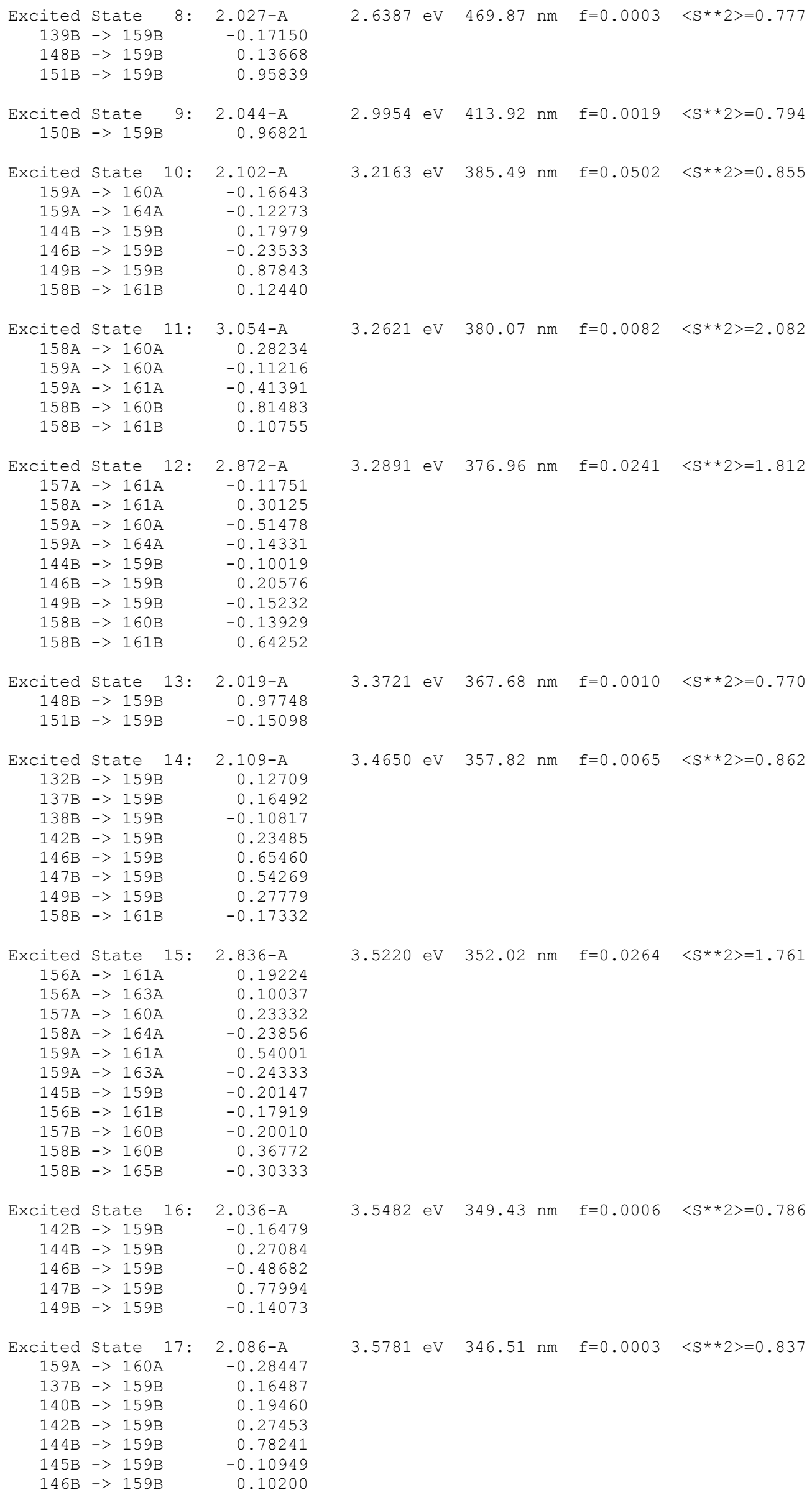




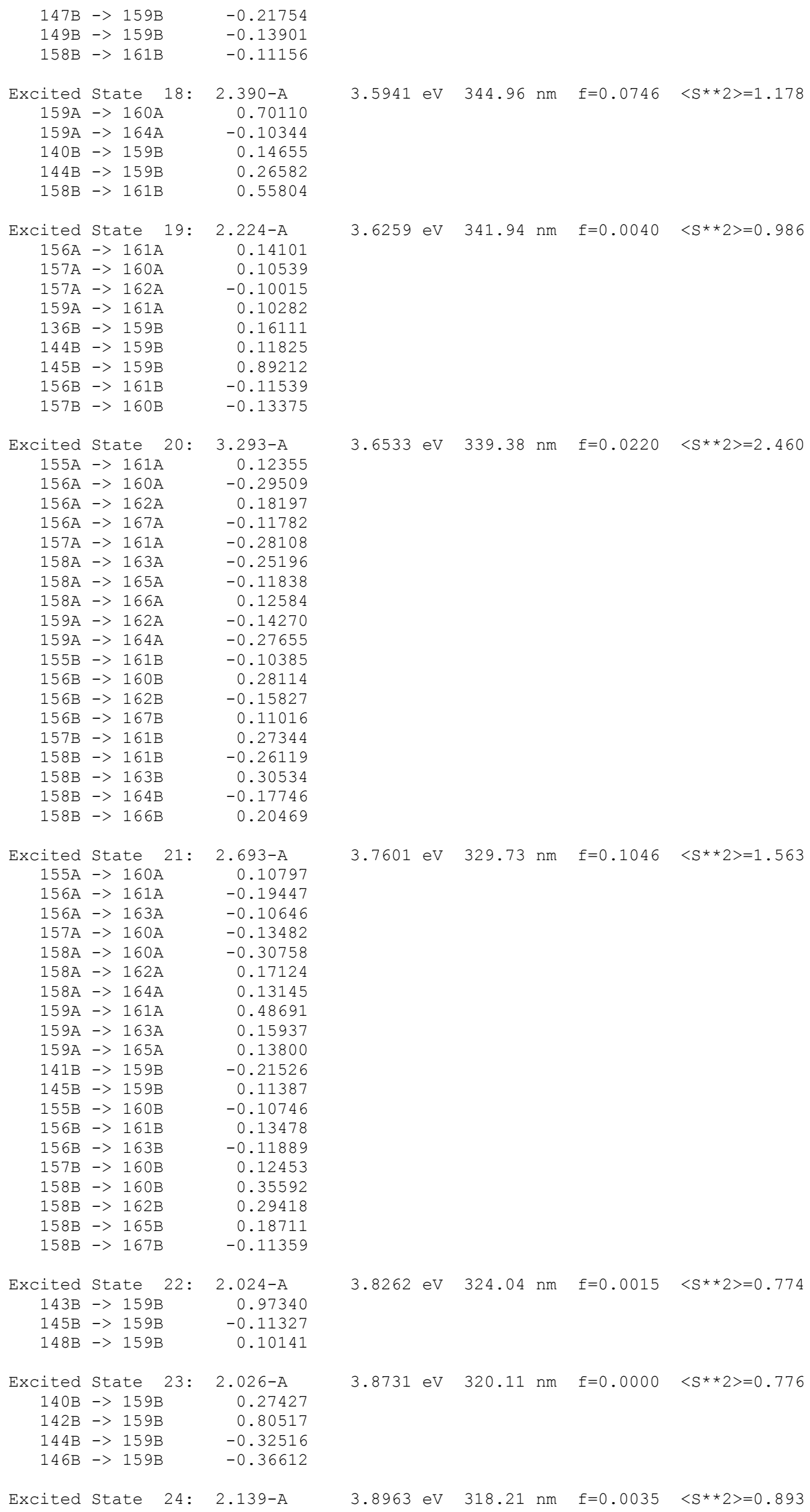




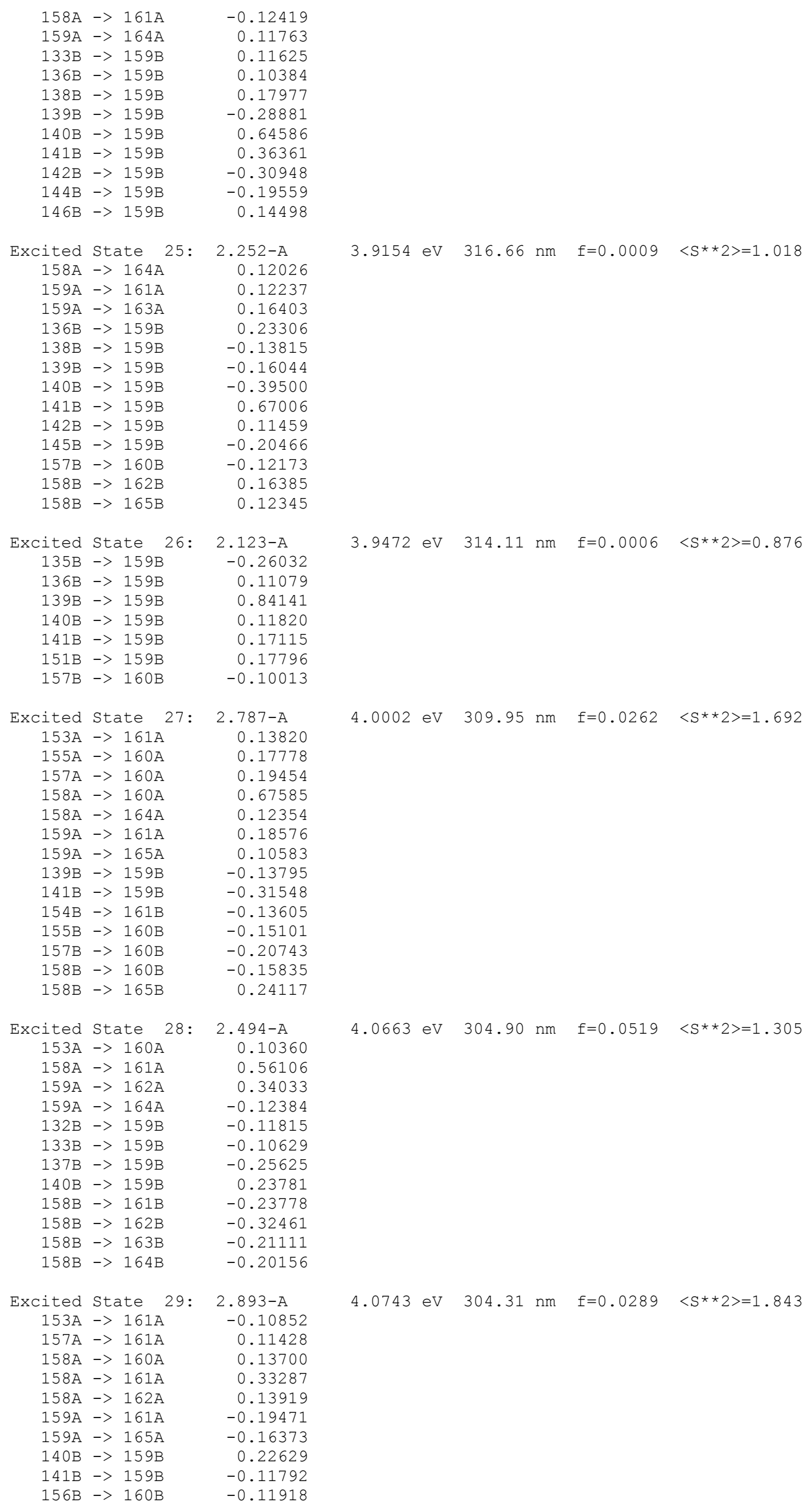

0.28881

0.64586

252-A

$-0.13815$

.67006

$123-\mathrm{A}$

0.11079

0.84141

0.11820

0.17796

.787-A

0.13820

.17778

0.18576

0.10583

$-0.13795$

.0 .131548

0.13605

0.15835

. 494-A

0.10360

0.56106

$-0.21111$

$-0.20156$

0852

0.33287

$-0.11918$ 
$\begin{array}{rr}157 \mathrm{~B}->161 \mathrm{~B} & -0.10410 \\ 158 \mathrm{~B}->161 \mathrm{~B} & -0.13307 \\ 158 \mathrm{~B}->162 \mathrm{~B} & 0.68618 \\ 158 \mathrm{~B}->165 \mathrm{~B} & -0.14843\end{array}$

Excited State 30: 2.264-A

$157 \mathrm{~A}->161 \mathrm{~A} \quad 0.10930$

$158 \mathrm{~A} \rightarrow 161 \mathrm{~A} \quad 0.20317$

$132 \mathrm{~B}->159 \mathrm{~B}-0.36125$

$137 \mathrm{~B}->159 \mathrm{~B} \quad 0.69570$

$138 \mathrm{~B}->159 \mathrm{~B}-0.18812$

$142 \mathrm{~B}->159 \mathrm{~B}-0.19363$

$146 \mathrm{~B}->159 \mathrm{~B} \quad-0.20264$

$158 \mathrm{~B} \rightarrow 162 \mathrm{~B} \quad-0.21031$

$158 \mathrm{~B}->163 \mathrm{~B} \quad 0.12687$
$4.0806 \mathrm{eV} \quad 303.84 \mathrm{~nm} \quad \mathrm{f}=0.0082 \quad<\mathrm{S} * \star 2>=1.031$ 